\title{
Investigation Of Ions Accelerated Through Electrostatic Menisci In An Inductively Coupled Plasma
}

\author{
David D. Caron \\ ddc0008@mix.wvu.edu
}

Follow this and additional works at: https://researchrepository.wvu.edu/etd

Part of the Plasma and Beam Physics Commons

\section{Recommended Citation}

Caron, David D., "Investigation Of Ions Accelerated Through Electrostatic Menisci In An Inductively Coupled Plasma" (2021). Graduate Theses, Dissertations, and Problem Reports. 10157.

https://researchrepository.wvu.edu/etd/10157

This Dissertation is protected by copyright and/or related rights. It has been brought to you by the The Research Repository @ WVU with permission from the rights-holder(s). You are free to use this Dissertation in any way that is permitted by the copyright and related rights legislation that applies to your use. For other uses you must obtain permission from the rights-holder(s) directly, unless additional rights are indicated by a Creative Commons license in the record and/ or on the work itself. This Dissertation has been accepted for inclusion in WVU Graduate Theses, Dissertations, and Problem Reports collection by an authorized administrator of The Research Repository @ WVU.

For more information, please contact researchrepository@mail.wvu.edu. 


\title{
INVESTIGATION OF IONS ACCELERATED THROUGH ELECTROSTATIC MENISCI IN AN INDUCTIVELY COUPLED PLASMA
}

\author{
David D. Caron \\ Dissertation submitted to the Eberly College of Arts and Sciences \\ at West Virginia University \\ in partial fulfillment of the requirements for the degree of \\ Doctor of Philosophy \\ in \\ Physics \\ Earl E. Scime, Ph.D., Chair \\ Paul A. Cassak, Ph.D. \\ Timothy N. Good, Ph.D \\ Weichao Tu, Ph.D. \\ Department of Physics and Astronomy \\ Morgantown, West Virginia \\ 2021
}

Keywords:

Plasma meniscus, sheaths, ion extraction, inductively coupled plasmas

Copyright 2021 David D. Caron 


\section{Abstract}

\section{INVESTIGATION OF IONS ACCELERATED THROUGH ELECTROSTATIC MENISCI IN AN INDUCTIVELY COUPLED PLASMA}

David D. Caron

Plasmas are used in semiconductor fabrication as they allow for very precise control over processes such as etching and doping. This is achieved by extracting a beam of ions from the plasma to interact with and modify the surface of a silicon wafer. However, conventional fabrication methods are reaching spatial limitations as semiconductor features reach the atomic scale. Therefore, in order to better control the fabrication processes and facilitate the transition to three-dimensional architecture, a greater understanding of ion beam formation is needed. Ion beams are extracted at the boundary between the Debye sheath and an externally applied potential, which forms a unipolar sheath. This boundary, known as the plasma meniscus, is dependent on source parameters and acts as an electrostatic lens for ions that traverse it. This allows for control of ion beam properties through the adjustment of the source parameters that affect the meniscus.

Presented here is an investigation into the plasma meniscus and the dependence of its topology on controllable source parameters. The plasma meniscus is formed by graphite extraction optics with a $5 \mathrm{~mm}$ square aperture for beam extraction. $12 \mathrm{~mm}$ from the aperture is an electrically isolated graphite wafer that is biased to different potentials. Laser induced fluorescence is employed to obtain ion velocity distribution functions: inside the inductively coupled plasma source, at the extraction aperture, and in the downstream ion beam. The use of the confocal telescope allows for first, non-perturbative measurements of ions inside an inductively coupled plasma source. The ion source power is varied $\left(P_{f}=1 \mathrm{~kW}, 2 \mathrm{~kW}, 3 \mathrm{~kW}, 4 \mathrm{~kW}\right)$ at different applied wafer bias voltages $\left(V_{b}=0 \mathrm{~V}, 1000 \mathrm{~V}, 2000 \mathrm{~V}, 3000 \mathrm{~V}\right)$.

Ion temperature, velocity, and relative density are calculated from the ion velocity distribution functions. The ions' speed increases as they travel through the source and form the beam. Additionally, there is a second population of ions that appears near the plasma meniscus. These ions form a beam halo, which hinders the creation of a uniform ion beam. This effect is mitigated at a sufficiently high bias voltage. 


\section{Acknowledgements}

To say that I am here because I stand on the shoulders of giants is an understatement. This journey has been supported by so many people throughout my life that it would take countless pages to properly thank them all.

First, I want to thank my family. To my brother Joe, who has always blazed the trail and led by example, thank you for your inspiration. To my mother, who taught us the value of a curious mind, thank you for everything. To my aunt Jackie, who always listened to me, thank you for your unconditional support. And to my grandfather Angelo, who showed me how to live a good life, I wish I could show you my accomplishments.

I also want to acknowledge Conway: Steve, Jay, Mike, John, Tim, Pratheek, and Matt. You all are the best and I am lucky to have friends like you. You have always been dependable and the times we spend together always reinvigorate me. Thank you all for being there for me.

I never would have made it to graduate school without the support of my undergraduate cohorts. Whether we were helplessly studying Classical Mechanics together or losing our minds late at night in the Electronics lab, we were in it together. Tyler, Joe, Kelly, Luke, and Cory, thank you for dragging me through when I couldn't do it alone.

I would also like to thank my previous advisors who helped make me the researcher I am today. Dr. Tyrel McQueen, who taught me to be an experimentalist. Dr. Angela Capece who introduced me to the fascinating world of plasma physics. Vlad Soukhanovskii, Marlene Patino, Luxherta Buzi, and everyone else at PPPL who helped me start my plasma physics career.

I cannot give enough thanks to the WVU machinists: J.R., Carl, and Doug. No matter how many times REVAN was brought back to them for welding, I never ran through their goodwill. Thank you for helping me construct my experiment and catching all my mistakes. There would be no experiment without you all.

Viola, you're a rockstar.

I also have to thank my group members, as this document would not exist without them. Derek, thank you for showing me how to be a researcher. Your rigor and dedication to science has served as an exemplar. John, your knowledge helped me get my feet under me and dive into experimental research. Thank you for sharing 
your knowledge of all things plasma, and music. Tommy, it is safe to say that there would be no dissertation without you. Your help was invaluable and I cannot thank you enough for helping me every step of the way. Whether it was turning knobs or bouncing ideas, you were always engaged and always an amazing friend. Cuyler, you were a great office mate and a great person. Thank you for teaching me that sometimes children need to be left behind. Andrew and Jake, it was a pleasure working with you in lab. I couldn't think of better conference companions and I know you both will be successful wherever you go. Regis, thank you for your hard work and modeling contributions to this document. Mitch, what else can I say other than, you've got the reach.

I wouldn't have been able to make it without my fellow graduate students. Amber, although we didn't leave together I am glad that we started together. You helped me keep my sanity and I am glad to have you as friend. Brent, thanks for being my study, video game, and outreach partner. It was a long road but thanks having my back. Andrew, without you graduate school would not have been nearly as fun. Although every time I go camping now I have flashbacks. Thank you three, without you graduate school would have been very different.

Earl, thank you for everything. It is suffice to say I would not be here without you. Your mentorship has been invaluable in shaping me in to a researcher. The experience and wisdom I have gained from you will be used throughout my career. 
Science isn't an exact science 


\section{Contents}

1 Motivation \& Context 1

1.1 The State of the Art . . . . . . . . . . . . . . . 1

1.2 Semiconductor Fabrication Plasmas . . . . . . . . . . . . . . . 2

1.2 .1 Doping ....................... 5

1.2 .2 Plasma Etching . . . . . . . . . . . . . . . . . . 8

1.3 Ion Extraction . . . . . . . . . . . . . . . . . . . 10

1.3 .1 Sheaths . . . . . . . . . . . . . . . 12

1.3.2 Current Density . . . . . . . . . . . . . . . . . . . 17

1.3 .3 Plasma Meniscus . . . . . . . . . . . . . . . . . . . . 18

1.4 Motivation .......................... 20

1.4.1 Neutral Beam Injectors . . . . . . . . . . . . . . . 22

1.4.2 3D Architecture . . . . . . . . . . . . . . 23

2 Experimental $\quad 26$

2.1 Inductively Coupled Plasmas . . . . . . . . . . . . . . 26

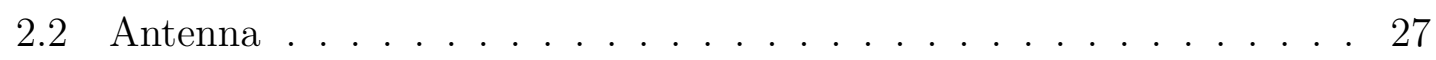

2.2.1 Transferred Power . . . . . . . . . . . . . . 29

2.2.2 Dissipated Power . . . . . . . . . . . . . . 30

2.3 Coupling Modes . . . . . . . . . . . . . . . . . . 32

2.3.1 Low Pressure .................... . . 33

2.3.2 High Pressure ................... 35 
2.3.3 E \& H Mode Transitions . . . . . . . . . . . . . . . . . . 36

2.4 REVAN Chamber . . . . . . . . . . . . . . . . . 38

2.4 Rf Circuit . . . . . . . . . . . . . . . . 39

2.4 Source ......................... 41

2.4.2.1 Slit Optics . . . . . . . . . . . . . . . 42

2.4.2.2 Aperture Optics . . . . . . . . . . . . . 43

2.4 .3 Wafer ........................ . . . . 44

2.4.4 Pressure Management . . . . . . . . . . . . . . . 45

2.4.5 Coordinate System . . . . . . . . . . . . . . . 47

$\begin{array}{lll}3 & \text { Diagnostics } & 49\end{array}$

3.1 Laser Induced Fluorescence _. . . . . . . . . . . . . . . . . . . . . . 49

3.1.1 Velocity Distribution Function . . . . . . . . . . . . 50

3.1 .2 Argon LIF . . . . . . . . . . . . . . . . . . . . . 53

3.1.3 Broadening Mechanisms . . . . . . . . . . . . . . . 54

3.1.3.1 Natural Linewidth . . . . . . . . . . . . . . . 54

3.1.3.2 Pressure Broadening . . . . . . . . . . . . 55

3.1.3.3 Zeeman Broadening ............. 55

3.1.3.4 Laser Power Broadening . . . . . . . . . . . . 56

3.1.3.5 Doppler Broadening . . . . . . . . . . . 56

3.1.4 Metastable Quenching . . . . . . . . . . . . . 58

3.2 Optics . . . . . . . . . . . . . . . . . . . 61

3.2 .1 Beampath ...................... 61

3.2 .2 Confocal System . . . . . . . . . . . . . . . 63

3.2 .3 Conventional Optics . . . . . . . . . . . . . . 66

3.3 Langmuir Probe . . . . . . . . . . . . . . . . . . . . . . . 68

3.3.1 Probe Construction . . . . . . . . . . . . . . . . 69 
3.3.2 Electronics....................... . . 70

3.3.3 Analysis ....................... 71

3.3.3.1 I-V Trace . . . . . . . . . . . . . . . . 71

3.3.3.2 Fluid Quantities .............. . . 73

3.4 Gaussmeter . . . . . . . . . . . . . . . . . . . . 77

$\begin{array}{llr}4 & \text { Results } & 80\end{array}$

4.1 Introduction . . . . . . . . . . . . . . . . . . 80

4.2 Angled ion beam from a slit . . . . . . . . . . . . . . 80

4.3 Ion beam extracted from a plasma meniscus . . . . . . . . . . . 84

4.4 Meniscus flow and temperature . . . . . . . . . . . . . . 91

4.5 LIF Measurements Inside the Source . . . . . . . . . . . . . . . 95

4.5.1 Bulk flow near the aperture from Two Population fits . . . . . 96

4.5 .2 IVDFs in the source . . . . . . . . . . . . . . . . . . . 98

4.5.3 Ions heat near the extraction region . . . . . . . . . . . 101

4.6 EEPFs . . . . . . . . . . . . . . . . . . . . . 102

4.6.1 Electron Density . . . . . . . . . . . . . . . . 103

4.6 .2 Electron Temperature . . . . . . . . . . . . . . . 104

$\begin{array}{lll}5 & \text { Conclusion } & 107\end{array}$

$\begin{array}{ll}\text { A E-mode } & 111\end{array}$

$\begin{array}{ll}\text { B Current Density } & 114\end{array}$ 


\section{List of Figures}

1.1 Integrated circuit fabrication . . . . . . . . . . . . . 3

1.2 Effect of wet and dry etching on different aspect ratios . . . . . . . . 4

1.3 Ion implanter energies . . . . . . . . . . . . . . 6

1.4 SEM image of a doped wafer . . . . . . . . . . . . . 7

1.5 Physical and chemical etch rate for silicon . . . . . . . . . . . 9

1.6 Sidewall processing . . . . . . . . . . . . . . . . . . . . 11

1.7 Debye boundary formation . . . . . . . . . . . . . . . . 13

1.8 Ion trajectory through a plasma meniscus f . . . . . . . . . 16

1.9 Different focusing geometries of the plasma meniscus _ . . . . . . . 19

1.10 Simulation of a negative ion beam halo . . . . . . . . . . . . 21

1.11 Schematic of a 3D gate . . . . . . . . . . . . . . . 23

1.12 Simulated ion beamlet extraction . . . . . . . . . . . . . 25

2.1 Types of ICP antennas . . . . . . . . . . . . . . . . . . 27

2.2 Induced ICP fields . . . . . . . . . . . . . . . . . . . . . . . 28

2.3 Simulated electric field inside an ICP . . . . . . . . . . . . . . . . 28

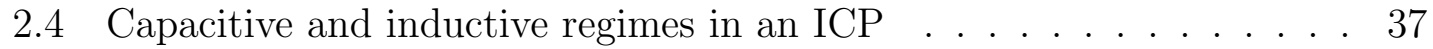

2.5 E and $\mathrm{H}$ mode transition hysteresis . . . . . . . . . . . . . 38

2.6 REVAN chamber . . . . . . . . . . . . . . . . . . . . 39

2.7 Matching network circuit diagram . . . . . . . . . . . . . . . . 40

2.8 Bisected view of REVAN . . . . . . . . . . . . . . . . . 41 
2.9 Slit extraction optics . . . . . . . . . . . . . . . . . 42

2.10 Aperture extraction optics . . . . . . . . . . . . . . 43

2.11 Biased wafer . . . . . . . . . . . . . . . . . . . . 44

2.12 Potential map of REVAN . . . . . . . . . . . . . . . 45

2.13 Pressure differential in REVAN . . . . . . . . . . . . . . . . 46

3.1 Ion properties obtained from an IVDF . . . . . . . . . . . . . 52

3.2 Partial Grotrian diagram of Ar II LIF scheme . . . . . . . . . . 53

3.3 Saturation broadening effects on IVDFs . . . . . . . . . . . . 57

3.4 LIF beampath . . . . . . . . . . . . . . . . . . . . . . 61

3.5 Confocal ray diagram . . . . . . . . . . . . . . . . . . . 63

3.6 Beam profiler images of the confocal system . . . . . . . . . . . 65

3.7 Model of the $2 \mathrm{D}$ optical stage $\ldots \ldots \ldots 7$. . . . . . . . . . . 67

3.8 IVDF with laser reflection . . . . . . . . . . . . . . . . . 68

3.9 Langmuir probe mounted on REVAN . . . . . . . . . . . . . . . . . 69

3.10 Interior image of Langmuir Probe . . . . . . . . . . . . . . . . 70

3.11 Rf compensated Langmuir probe . . . . . . . . . . . . . . 71

3.12 Langmuir Probe signal filtering . . . . . . . . . . . . . . . 72

$3.13 \mathrm{I}-\mathrm{V}$ trace in REVAN . . . . . . . . . . . . . . . . . . . . 74

3.14 Electron current from a Langmuir probe . . . . . . . . . . . . . . 76

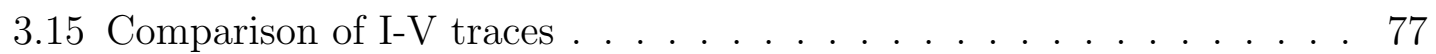

3.16 Gaussmeter probe configurations . . . . . . . . . . . . . . 79

4.1 Extraction through slit optics . . . . . . . . . . . . . 81

4.2 Angled ion beam density map and flow field . . . . . . . . . . . . . 82

4.3 REVAN measurement regions . . . . . . . . . . . . . . . . . 84

4.4 REVAN ion beam, no bias . . . . . . . . . . . . 86

4.5 REVAN ion beam, $3000 \mathrm{~V}$ bias . . . . . . . . . . . 87 
4.6 Properties of a laminar beam . . . . . . . . . . . . . . . . . 88

4.7 Ion beam velocity and temperature without a bias . . . . . . . . . . 89

4.8 Ion beam velocity and temperature with a $3000 \mathrm{~V}$ bias . . . . . . . 90

4.9 Ion flow through the aperture . . . . . . . . . . . . . . 92

4.10 Ion temperature in the aperture . . . . . . . . . . . . . . . 94

4.11 Meniscus expansion . . . . . . . . . . . . . . . . . . . 95

4.12 Comparison of Maxwellian and non-Maxwellian IVDFs . . . . . . . . 96

4.13 Maxwellian vs. Non-Maxwellian fits . . . . . . . . . . . . . . . . . 97

4.14 Bulk Flow inside REVAN . . . . . . . . . . . . . . . . . . . . . 98

4.15 Comparison of high-energy features in IVDFS . . . . . . . . . . . . 99

4.16 Full REVAN IVDFs . . . . . . . . . . . . . . . . . . 100

4.17 Ion temperatures inside REVAN . . . . . . . . . . . . . . . . . . . 102

4.18 Calculated electron density . . . . . . . . . . . . . . . . . 103

4.19 Calculated electron temperature . . . . . . . . . . . . . 105

A.1 Dielectric barrier deposition . . . . . . . . . . . . . . 112 


\section{List of Tables}

4.1 Debye lengths in REVAN . . . . . . . . . . . . . . . . . 105

B.1 Comparison of experimental current densities . . . . . . . . . . 115 


\section{Chapter 1}

\section{Motivation \& Context}

\subsection{The State of the Art}

The use of plasmas in surface modification and processing is the standard in the semiconductor industry and a crucial technology for applications such as aerospace, biomedicine, and toxic waste management [1-5]. Plasma is desirable for these applications because it is highly reactive, its properties and applications are mutable depending on the constituent gas, and the interactions between charged particles and electromagnetic forces allow for control. This makes it appealing for surface modification and plasma-material interactions where micron-scale features are necessary. Perhaps the most important application of plasma in industry is the use of plasma for large-scale production of integrated circuits (IC).

IC fabrication is an extensive and delicate operation, but it is essentially three repeating actions: first a film is grown on a silicon wafer, then the wafer is doped, and then finally it is etched and cleaned. To create features on the wafer, photolithography is used. This process begins with a photosensitive chemical photoresist layer deposited on the wafer. Then, exposure to UV light through an optical mask removes select regions of the photoresist and patterns the wafer. Material under the 
photoresist is not affected by etching and doping, thus allowing for the shaping of structures and features. Figure 1.1 shows how the repeating cycle of growth, doping, and etching is used with photolithography to create features on a silicon wafer.

In 1965, Gordon Moore remarked that the complexity for minimum component cost doubled every two years and hypothesized that this trend is likely to continue for at least the next ten years [6]. For nearly fifty years this trend held. A combination of cheaper manufacturing cost, coupled with the ability to pack features more densely on a single IC, led to a boom in computer processing power and the popularization of the concept of Moore's Law [7]. Many technical advances were necessary to keep up with Moore's Law while maintaining a low cost of production.

One major advancement in the industry was from the transition from liquid chemicals to plasmas for etching and doping. This change, from "wet" techniques to "dry" techniques, was necessary to yield the higher aspect ratios necessary for denser chips. Aspect ratio is defined as a feature's height over width, so a higher ratio would allow for more features to be packed on an IC. Using a chemical solution in wet etching causes etching to occur isotropically. Since the etch rates are the same in the horizontal and vertical directions, it is hard to control this process which leads to undercutting, a defect shown in Fig. 1.2. At low aspect ratios this defect is often ignored, given that it is within allowable tolerances for chip production [8]. But, as the aspect ratio increased, wet etching was insufficient for industry needs. Another advantage to using plasmas over wet chemicals is that less hazardous by-products are produced. Additionally, if all fabrication processes are dry, the wafer never has to leave vacuum, reducing the chances of defects.

\subsection{Semiconductor Fabrication Plasmas}

Plasmas were not seriously considered for semiconductor processing until Coulter and Keller [9] patented a new type of a planar inductively coupled plasma (ICP) 


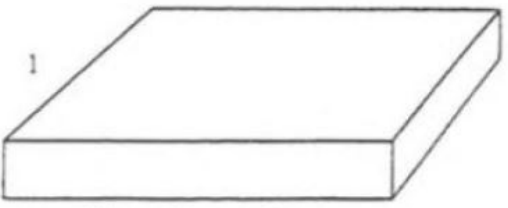

n-Type silicon wafer

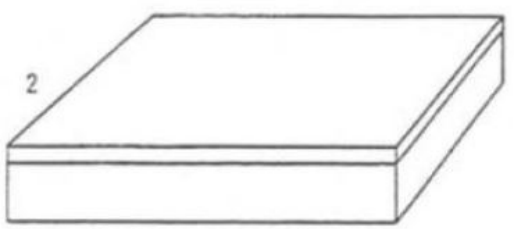

Layer of silicon oxide is formed

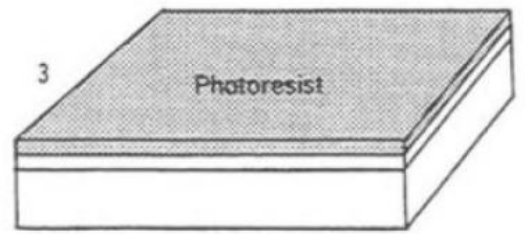

Photoresist is applied

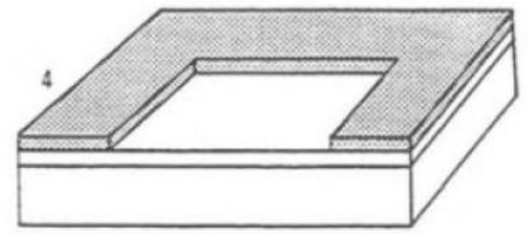

Photoresist is exposed and developed

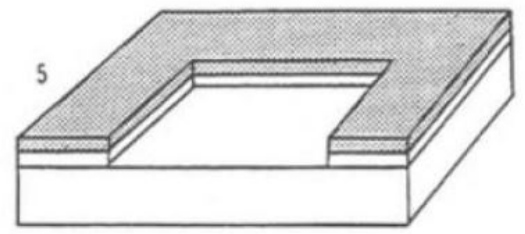

Silicon oxide in base regions

is etched away

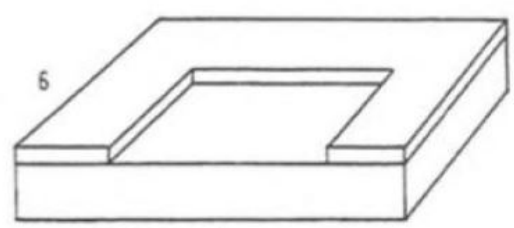

Photoresist is removed

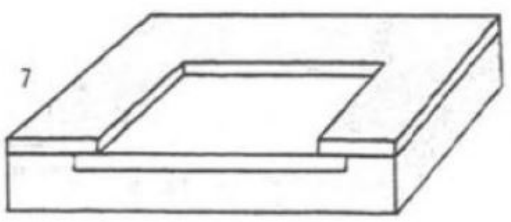

p.Type base is diffused in

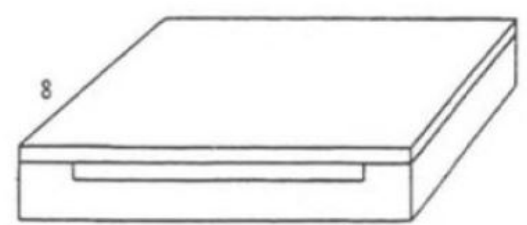

Surface is reoxidized

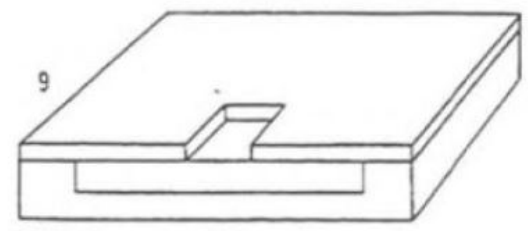

Oxide is removed for emitter region

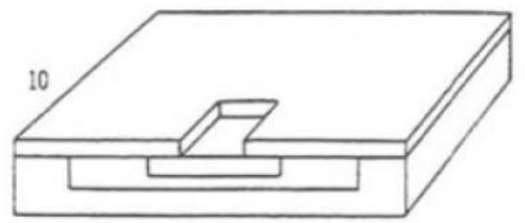

n-Type emitter is diffused in

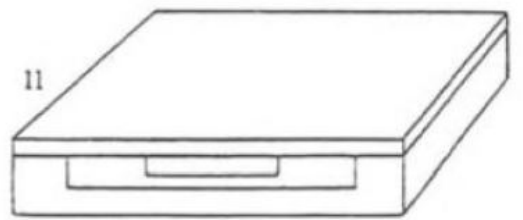

Surface is reoxidized

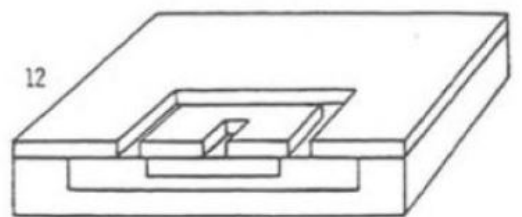

Base and emitter contact

areas are etched out

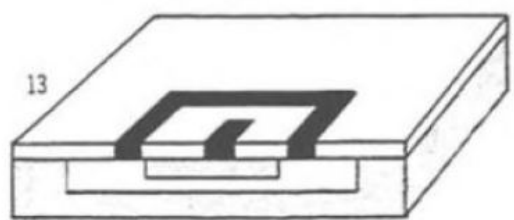

Aluminum contacts are evaporated on

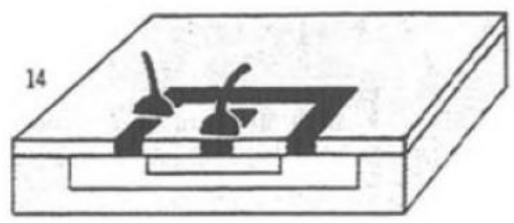

Connectors are then attached to the contact areas

Figure 1.1: Diagram of the iterative steps needed to produce a single p-n junction on a silicon wafer. These features are created by a repeated cycle of growth, doping and etching. Reprinted with permission from Chemical \& Engineering News (C) 1970 American Chemical Society). This image appeared on page 47 of the Nov. 30, 1970, issue (vol. 48, issue 50) 


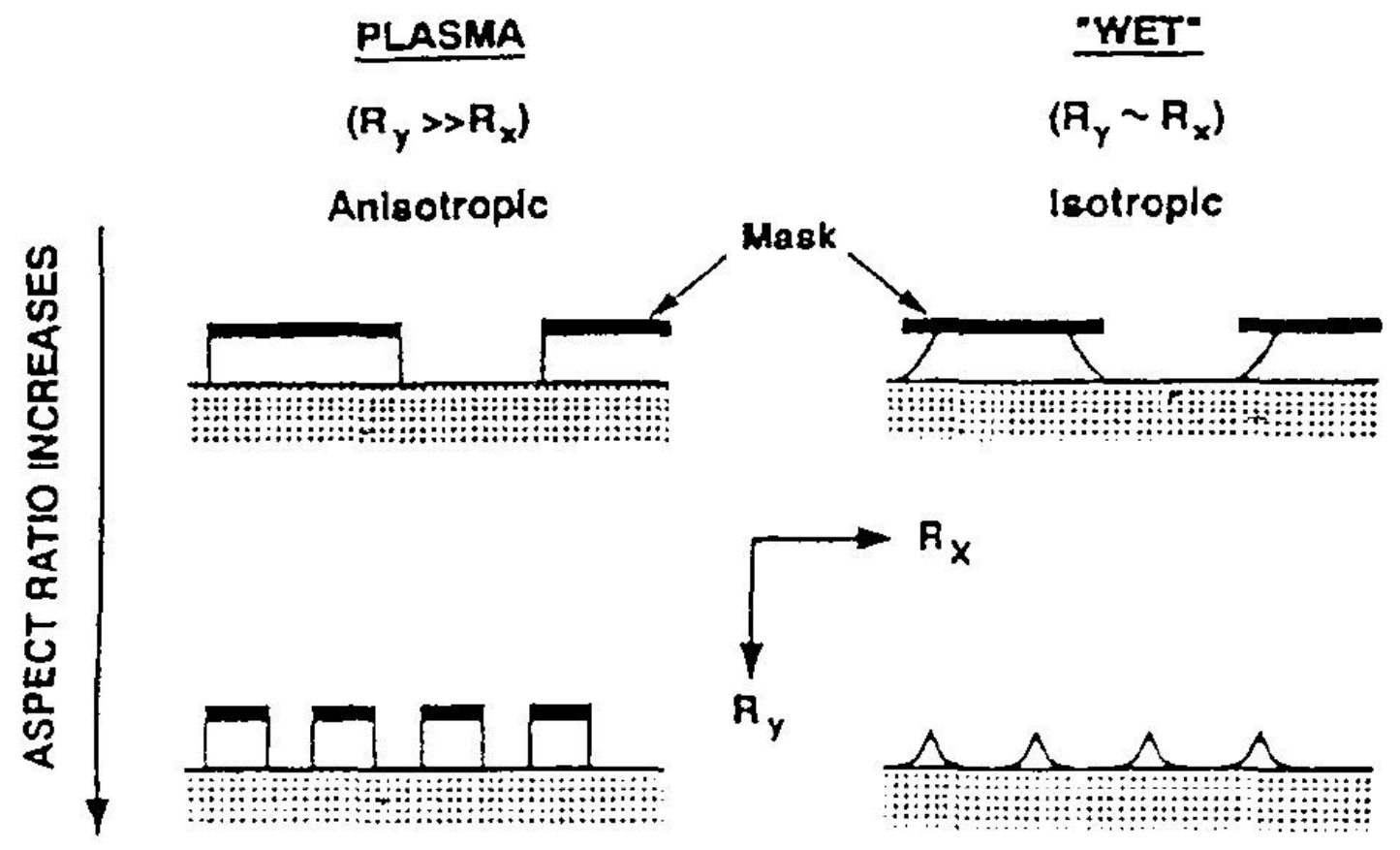

Figure 1.2: Illustration of the aspect ratio dependent failure mode called undercutting. A wet etch can totally destroy a semiconductor's feature by circumventing the mask. Plasmas do not suffer this drawback due to the high directionality of their etch. Reproduced with permission from Ref. [8], all rights reserved.

source. This design generates a large volume of uniform plasma for semiconductor applications. By uniformly scaling the plasma to a large volume, planar ICPs were able to keep up with industry goals for throughput, or the number of completed units per unit time. Throughput is a driving factor behind semiconductor design. Therefore, to maximize efficiency, semiconductor manufacturers seek fabrication methods with high throughput [10].

While the efficiency of a processing technique is based on the throughput, the quality of the processing plasma is represented by anisotropy, selectivity, and uniformity [8]. Anisotropy is perhaps the most important parameter for a reliable etch of small features. While plasmas excel in this regard, they cannot produce totally anisotropic beams, i.e., beams with all velocities in a single direction. During processing of a silicon wafer, a beam of ions is extracted from the plasma and directed towards the wafer. This beam will have an innate distribution of ions with differ- 
ent angles with respect to the beam's direction of propagation, which is dependent on the extraction geometry. Ions will also scatter off neutrals, from the unionized gas or produced from recombination at the wafer, thus reducing the beam's anisotropy $[8,11]$. Selectivity is the relative etch rates of the plasma acting on different materials. Since a component of the plasma etch is physical bombardment, plasmas have low selectivity and will etch a photoresist as well as the wafer surface. Uniformity is how consistent the rate of modification of the wafer is over the processing area. The extracted ion beams used in etching applications are planar beams that are wider than the wafer. This gives high uniformity across the wafer, which is scanned perpendicular to the beam in order to process the entire surface. However, since the extracted beam is planar, it will only uniformly process two-dimensional structures, making etching and doping of the trenches and the sidewalls of features difficult.

An efficient plasma, however, is not always congruent with high throughput. There is usually a trade-off between processing speed and control. Accelerating ions to high velocities will increase the ion flux and anisotropy at the wafer, since the ions will have less time to deviate during their time of flight, but the etch rate becomes difficult to control. Large processing areas increase throughput, but will only have good uniformity for planar topology, limiting the practical geometry of features. Balancing production, to keep costs low, and complexity, to follow Moore's Law, is at the core of semiconductor plasma innovation. Below is a brief overview of how plasmas are used in the fabrication process.

\subsubsection{Doping}

ICs are fabricated on wafers made of silicon, which is in the periodic group XIV. Therefore silicon, like carbon, prefers a lattice structure of four covalent bonds and a full valence shell. This also means that pure silicon is an insulator. In order to create a semiconductor from an insulated wafer, controlled imperfections are added 
to the lattice structure. These impurities are called dopants and are typically atoms that are one periodic group higher or lower than the constituent elements of the wafer.

For silicon, if the dopant atom is from periodic group XV (phosphorous, arsenic, or antimony) then the impurity will donate an electron to the silicon lattice. This creates an n-type semiconductor. If the impurity is from periodic group XIII (boron, gallium, or indium) then it will have one less electron, creating a quasiparticle known as a hole. This lack of an electron creates a p-type material.

The introduction of a hole or extra electron changes the energy level structure of the lattice. These imperfections moderate the band gap between the valence and conduction band. The energy required to jump this gap is controlled by the type and amount of dopant implanted in the lattice. This makes doped materials act as

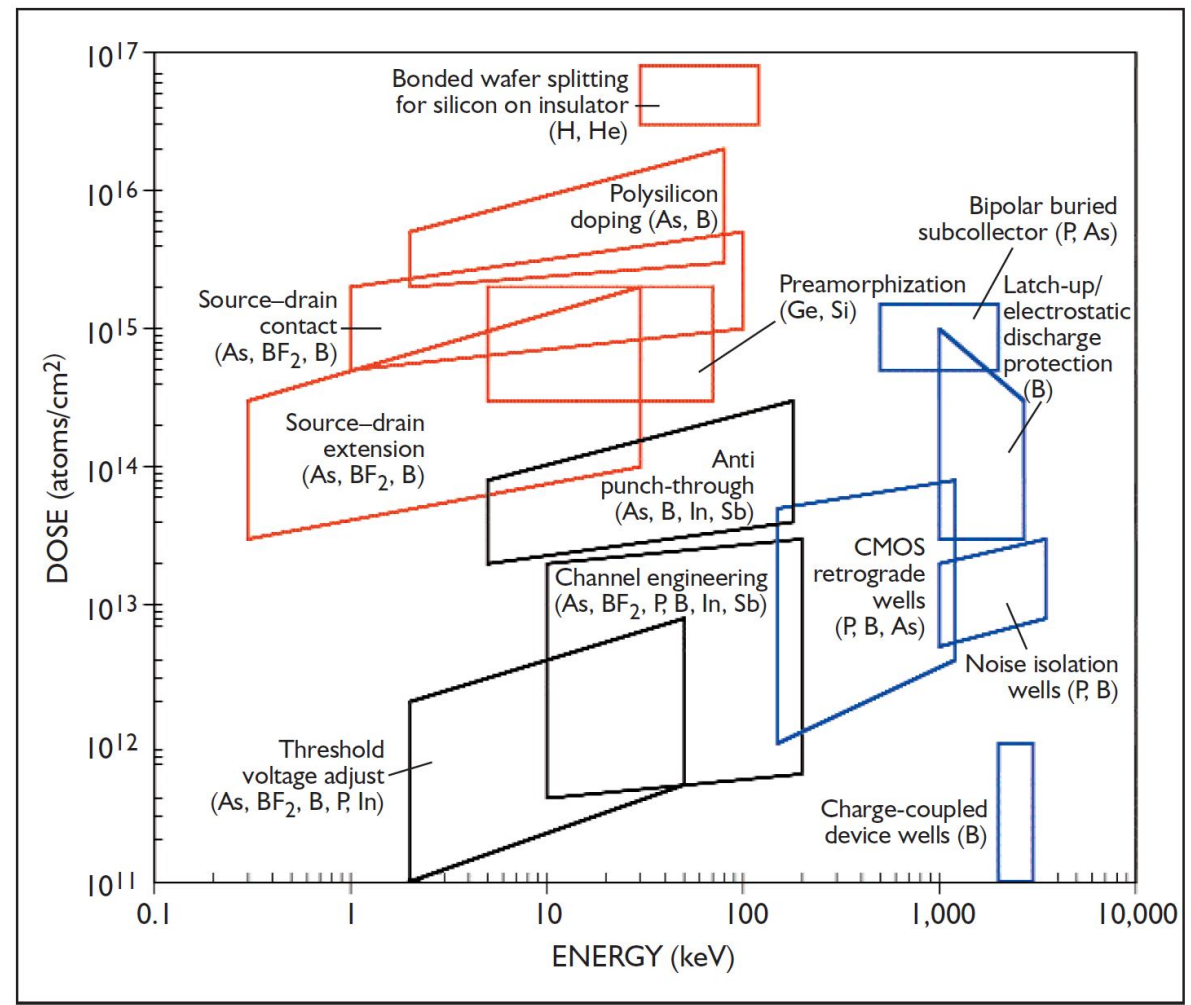

Figure 1.3: Diagram of the different ion energies required for different doping methods. Dose rate is dependent on ion energy, resulting in sloped borders. Due to the wide range of energies needed, many different implanters are needed (designated by different colors) to fabricate a CMOS. Reproduced with permission from Ref. [12], all rights reserved. 

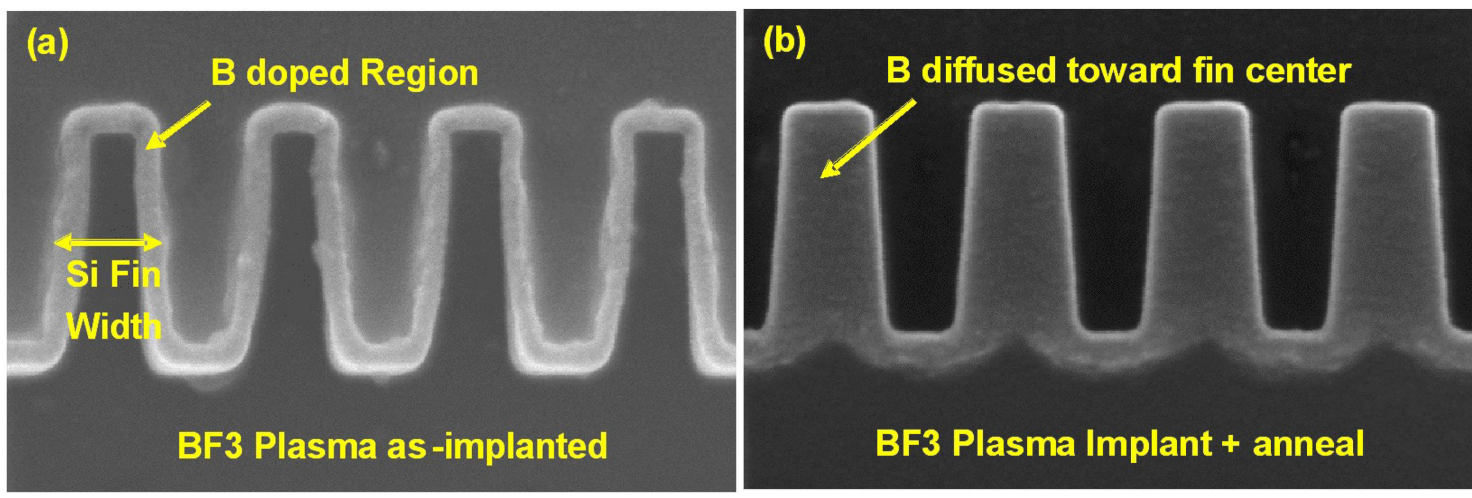

Figure 1.4: Image of a wafer using a cross section secondary electron microscope. a) The wafer after PLAD and b) the wafer after annealing. Reproduced with permission from Ref. [15], all rights reserved.

a gate. When applied voltages exceed the band gap, electrons enter the conductive band. A lower voltage produces no effect. The p-type and n-type regions can also be placed next to each other and combine to form junctions, the basis of diodes. Therefore, the quality of the electronic components of features is dependent on the level of control provided by the doping method.

One method to achieve dopant implantation is to use a high energy ion implanter. This method achieves good throughput due to the large ion current implanters produce [13]. However, ion implanters are not a "one-size-fits-all" for ion implantation, as shown in Fig. 1.3. The process for a complementary metal-oxide-semiconductor (CMOS) requires as many of 35 different types of implantation across a large range of energies [12]. Since extracted current is related to throughput, implantation at lower energies requires an increase in implantation time [14].

Other methodologies for dopant implantation are called plasma doping (PLAD) or pulsed plasma doping (PPLAD). For these techniques, the wafer is negatively biased while immersed in a dopant gas. The wafer becomes a cathode and ignites the plasma. The dopant ions are pulled from the plasma, accelerated through the wafer's sheath, and implanted in the wafer. P/PLAD delivers both a high ion flux and uniformity [16]. Figure 1.4 shows the even thickness and total coverage of the dopant obtained with this technique. However, PLAD is limited to doping depths in 
the tens of nanometers range and will dope the entire wafer, meaning that additional lithographic masks are needed to achieve the necessary selectivity [14,17].

\subsubsection{Plasma Etching}

A second application of plasmas for semiconductor manufacturing is plasma etching. This technique is currently the standard etching method for many semiconductor applications. This is due to the high degree of control plasmas offer. The benefits of plasma are readily apparent when it is compared to the previously used wet etching technique. Additionally, plasma etch rates are much easier to control since they are controlled electrically, while chemicals need to be washed away.

The process of plasma etching has both a physical and chemical component. Physically, ion bombardment can cause sputtering of a material through high energy impact. This is performed in a similar way to ion implantation (using a long beamline with multiple acceleration grids). This type of plasma etching has good anisotropy and uniformity, but is not very selective, sputtering all material it comes in contact with. Furthermore the etch rate from ion bombardment is low, resulting in poor throughput [18]. Chemically, plasma is used to generate radicals in an etchant gas to remove material. The mechanism for a chemical etching reaction is:

1. Creation of reactive species.

2. Adsorption of species to the material surface.

3. Chemisorption to produce a volatile molecule.

4. Desorption of molecule and removal from system.

This method produces generally good etch rates, but at the cost of the anisotropic nature inherent in an ion beam. However, if an ion beam is used in combination with a reactive gas, the best of both techniques are obtained. Figure 1.5 shows 
the relative etch rates of the plasma etching methods both separately and together. Using an ion beam to assist a reactive plasma can increase the etch rate tenfold. This is because the ion bombardment provides extra energy that assists weakly volatile molecules in desorption [19].

While plasma etching is well suited to semiconductor manufacturing, it does have drawbacks. The use of an etching plasma means that the wafer is totally immersed in the plasma, creating some isotropic etching all around the wafer. Additionally, the ion beam will have some unavoidable angular distribution of its ions, thus increasing the etch in unwanted directions. Ion beams also suffer aspect ratio dependent etching (ARDE), or microloading, where small aspect ratio features are etched slower because rising etched material near the endpoint will interrupt ion bombardment [20]. Currently, $35 \mathrm{~nm}$ trenches can be produced with an aspect ratio of 120:1 [21], but these issues prevent plasma etching from achieving the precision

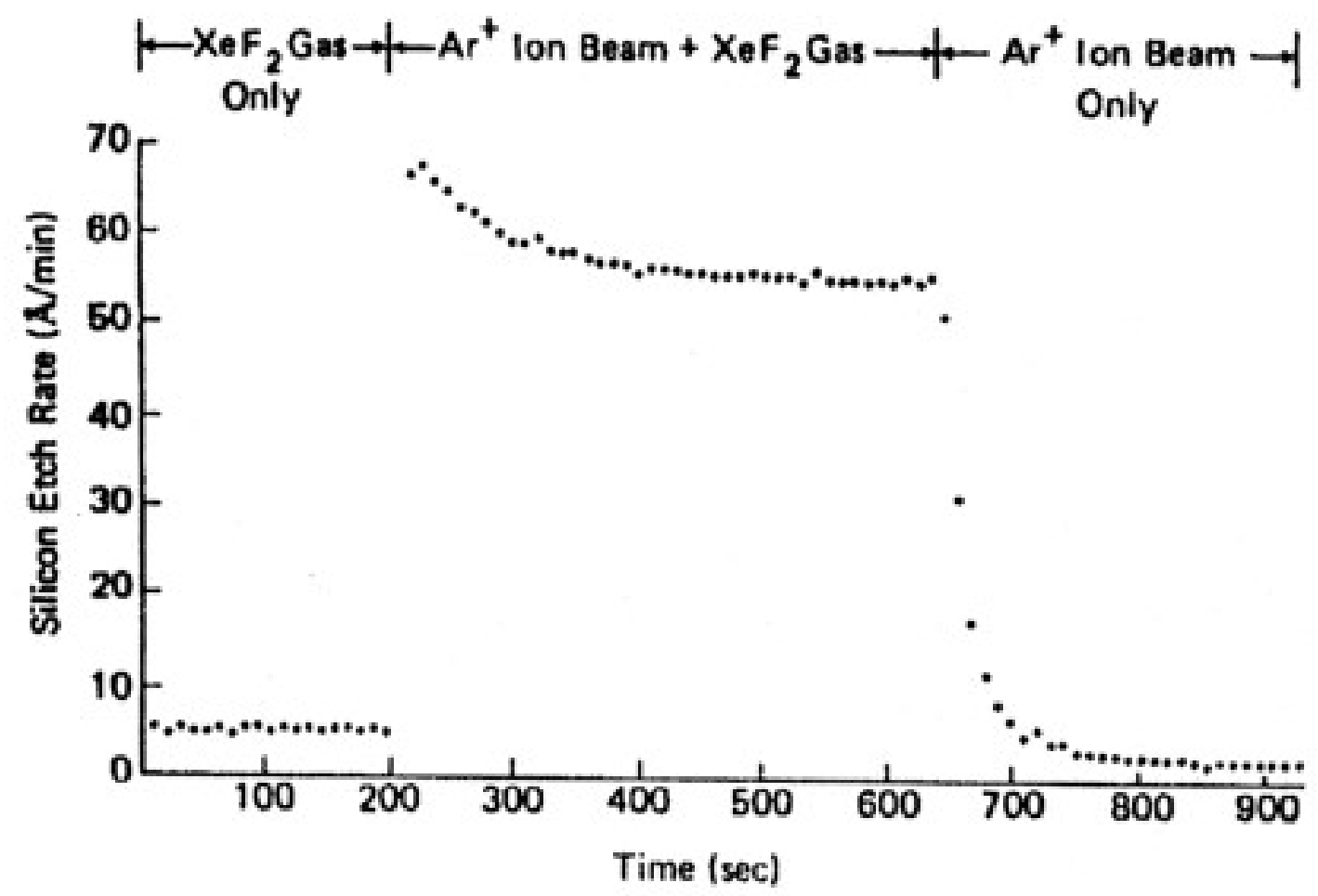

Figure 1.5: Silicon etch rate for different methods. The etch is chemical $\left(\mathrm{XeF}_{2}\right)$ in the beginning, both chemical and physics $\left(\mathrm{Ar}^{+}+\mathrm{XeF}_{2}\right)$ in the middle, and purely physical at the end $\left(\mathrm{Ar}^{+}\right)$. Reproduced with permission from Ref. [19], all rights reserved. 
needed for even higher aspect ratio features.

\subsection{Ion Extraction}

Applications like ion implanters and ion etching require an ion beam. This beam is extracted from a plasma source and accelerated to the target surface, typically by a series of electrostatic potentials. These ions, extracted from a bulk plasma, will travel through unique regions that will determine their energy and trajectories at the wafer. The plasma is separated from the extraction region, where the ion beam propagates, by the extraction optics. This surface must bound the plasma, with minimal contamination, determine the shape of the extracted beam, and set up the potential structure that extracts ions from the bulk plasma. In semiconductor manufacturing, the silicon wafer is often held at a negative bias to create an extraction potential and accelerate ions out of the plasma. Control over focusing and accelerating ion beams is a field of active research for industry [22].

The boundary though which the ions exit the plasma acts as an ion emitter, similar to a thermionic cathode that is heated to emit electrons. In contrast to thermionic emitters, this ion emitter produces ions with coherent trajectories rather than random emission. The beam resulting from an ion emitter is described by considering an extracted ion beam with an applied potential along its axis. This will cause an acceleration parallel to the ion beam such that [22],

$$
a=\frac{e E}{m}\left(\frac{V_{2}-V_{1}}{s}\right)
$$

where $a$ is the ion's acceleration, $e$ is the elementary charge, $E$ is the electric field at the emitting surface, $m$ is the mass of the ion, $V_{2}$ and $V_{1}$ are two equipotential surfaces a distance $s$ apart. Assuming that the particles exit the extraction optics at some angle from the normal, the beam has two components; the component along 


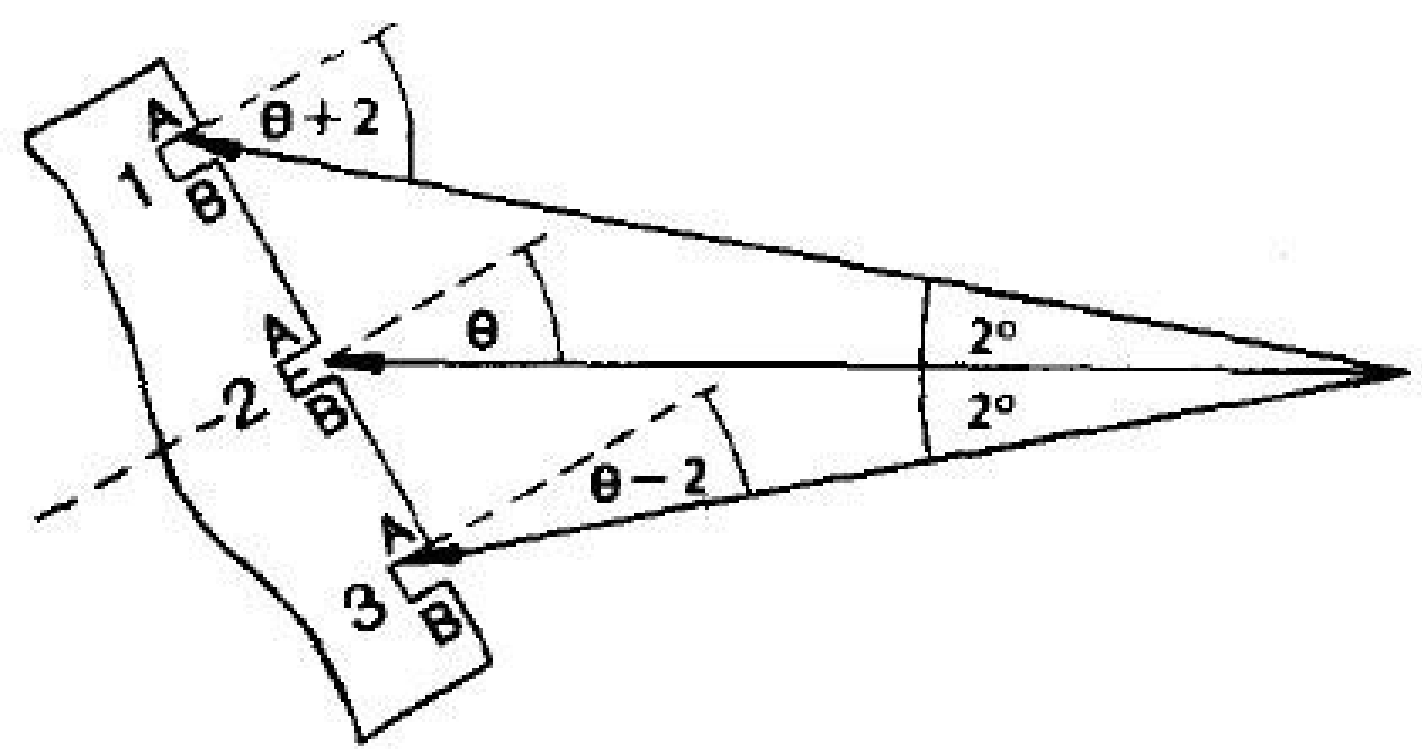

Figure 1.6: Image of current sidewall and trench doping methods. The wafer must be tilted with respect to the extracted ions so that the planar beam can access the vertical walls. The beam will strike different points of the trench depending on the location of the feature and angle of the wafer. Reproduced with permission from Ref. [23], all rights reserved.

the beam, $v_{1} \cos \theta$, and a transverse component, $v_{1} \sin \theta$, where $\theta$ is the angle of the ion's trajectory with respect to the beam normal. After some time $t$ the particle displacement is

$$
\begin{aligned}
& X=v_{1} \cos (\theta) t+\frac{e}{2 m} \frac{V_{2}-V_{1}}{s} t^{2}, \\
& Y=v_{1} \sin (\theta) t .
\end{aligned}
$$

Here $v_{1}$ is the velocity of the ion, $\theta$ is the angle of the ion with respect to the normal, and $m$ is the mass of the ion. Rearranging Eqn. 1.2 and Eqn. 1.3 yields a parabolic trajectory for the ions of the form,

$$
X=\frac{q}{2 m} \frac{V_{2}-V_{1}}{s} \frac{Y^{2}}{v_{1}^{2} \sin ^{2} \theta}+Y \cot \theta
$$

Equation 1.4 shows how the ion beam is extracted uniformly from the plasma. However, their trajectories will always have some component in the transverse direction unless the ions are extracted perfectly along the beam normal. Because 
of this, current techniques have poor uniformity when dealing with the non-planar structures, making the doping and etching of sidewalls and trenches an issue. To target these regions involves tilting the entire wafer to some angle with respect to the extracted beam (Fig 1.6). However, at higher aspect ratios the angle needs to be very highly oblique, which causes the feature's walls to be blocked by other, nearby features [23].

After the beam is extracted, a number of techniques are used, such as time of flight [24], absorption spectroscopy [25,26], Faraday cups [27], and infrared imaging [28], to measure beam properties. While it is easy to measure the ion beam after extraction, measuring ion properties inside the source at the emitting surface is significantly more difficult. To the best of the author's knowledge, there are no direct measurements of the emitting surface for an ICP ion beam achieved before this work. This is mostly due to the restrictive geometry of the sources. Ion beam apparatuses provide very limited optical access, have large enough densities and temperatures to damage probes, and the opening in the extraction optics, referred to here as the aperture, is restrictively small. However, it is the extraction region inside the plasma that is critical to downstream ion properties. This makes the investigation of the emitting region a high priority for devices that use extracted ions.

\subsubsection{Sheaths}

When a plasma comes in contact with a boundary, a transition region connecting the boundary to the bulk plasma is formed, known as the sheath. In this region the much lighter electrons reach the boundary first, giving it a negative potential relative to the plasma. This creates a layer at the edge of the plasma with a potential structure that will accelerate ions normal to the boundary. Known as the Debye sheath, this structure has a characteristic length called the Debye length $\lambda_{D}$ given 


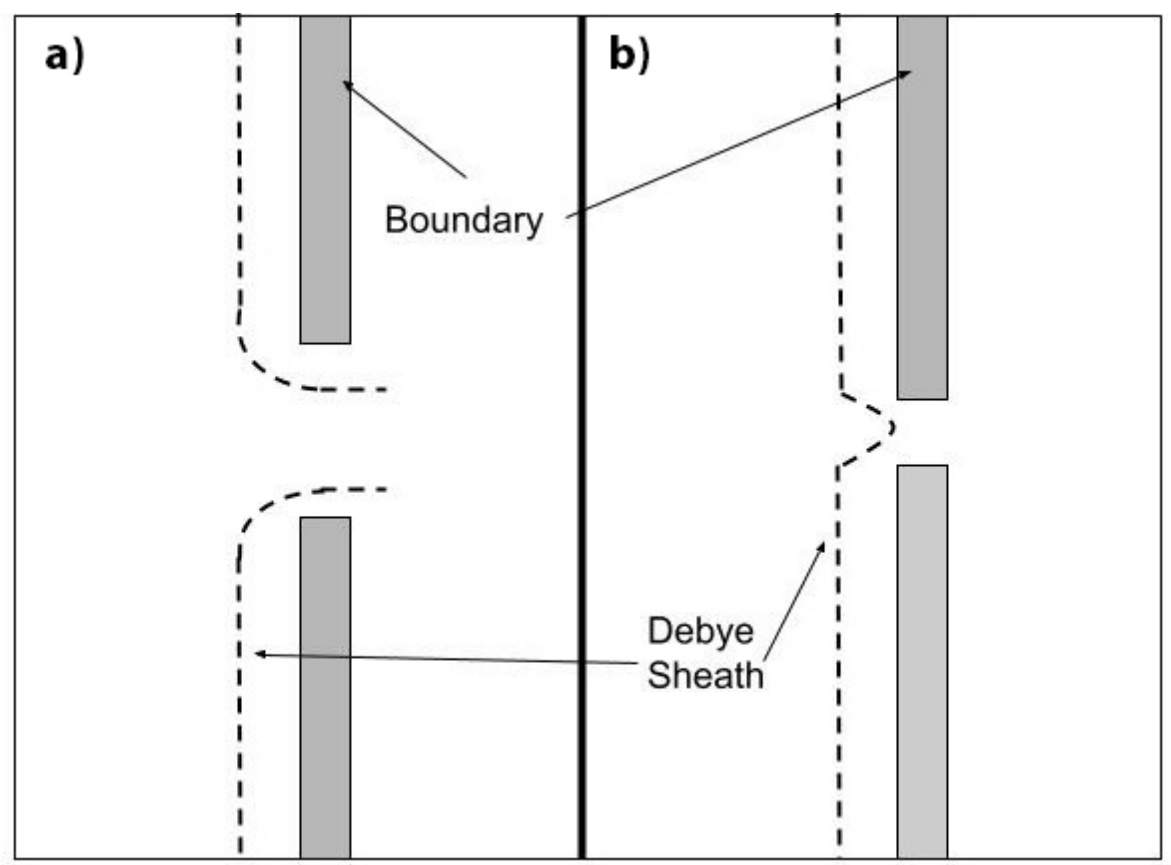

Figure 1.7: Diagrams illustrating the dependence of aperture size on the Debye sheath. a) Shows the typical contours of the Debye sheath through an opening. b) Illustrates how a smaller aperture size will create a continuous Debye sheath boundary.

by

$$
\lambda_{D}=\sqrt{\frac{\epsilon_{\mathrm{o}} k_{B} T_{e}}{n e^{2}}} .
$$

Here, $\epsilon_{\circ}$ is the permittivity of free space, $k_{B}$ is the Boltzmann constant, $T_{e}$ is the electron temperature, and $n$ is the plasma density. A Debye sheath typically extends into the plasma several $\lambda_{D}$. A detailed study of this plasma phenomena is found in Reference [29] or Reference [30].

A Debye sheath will form on the extraction optics for an ion beam source. The sheath formed by the extraction optics far from the extraction aperture does not affect the extracted ion beam and is therefore ignorable. However, at the aperture the sheath will follow the physical contours of the extraction optics. If the aperture size is small enough (on the order of a few Debye lengths) then the sheath can bridge the gap between two sides of the aperture and create one continuous sheath over the aperture, as shown in Fig. 1.7. 
Physically, the sheath balances the particle fluxes throughout the transition region. The particle dynamics along the path of extraction, $z$, are described by a two fluid hydrodynamic model [31]:

$$
\begin{aligned}
\frac{\partial}{\partial z}\left(n_{e} v_{e}\right) & =0 \\
\frac{\partial}{\partial z}\left(n_{i} v_{i}\right) & =0 \\
\frac{\partial}{\partial z}\left(m_{e} n_{e} v_{e}^{2}\right) & =-\frac{\partial}{\partial z}\left(n_{e} T_{e}\right)+q n_{e} \frac{\partial \phi}{\partial z} \\
\frac{\partial}{\partial z}\left(m_{i} n_{i} v_{i}^{2}\right) & =-\frac{\partial}{\partial z}\left(n_{i} T_{i}\right)+q n_{i} \frac{\partial \phi}{\partial z} \\
\frac{\partial^{2} \phi}{\partial z^{2}} & =4 \pi e\left(n_{e}-n_{i}\right)
\end{aligned}
$$

where $\phi$ is the potential, $q$ is the charge of the particle, and the subscripts $e$ and $i$ denote electrons and ions, respectively. These equations are commonly simplified using low temperature assumptions. First, the kinetic energy of the electrons is small compared to ions due to their mass difference. Therefore, the left hand side of Eqn. 1.6c is set equal to zero, giving a Boltzmann distribution for electron density,

$$
n_{e}=n_{\infty} \exp \left(\frac{e \phi}{T_{e}}\right)
$$

The term $n_{\infty}$ is used to represent the unperturbed density value far from the sheath in the bulk of the plasma. Secondly, the ion temperature under these assumptions is typically much less than the electron temperature $\left(T_{i} \ll T_{e}\right)$ and therefore is neglected. This simplifies Eqn. 1.6d, and together with Eqn. 1.6b gives an expression for the ion velocity,

$$
v_{i}=\sqrt{v_{0}^{2}-2 e \phi / m_{i}}
$$

where $v_{0}$ is the ion velocity in the bulk of the plasma, far from the sheath's potential. 
An expression for ion density is derived from Eqn. 1.8 using Eqn. 1.6b,

$$
n_{i}=\frac{n_{0} v_{0}}{\sqrt{v_{0}^{2}-2 e \phi / m_{i}}}
$$

Where $n_{0}$ indicates the ion density when there is no potential, in the bulk plasma. To simplify the derivation, dimensionless variables for normalized length, potential, and speed,

$$
\xi=\frac{z}{\lambda_{D}}, \quad \chi=\frac{e \phi}{T_{e}}, \quad u=\frac{v}{c_{s}}
$$

are used where $c_{s}$ is the ion sound speed,

$$
c_{s}=\sqrt{\frac{T_{i}+T_{e}}{m_{i}}} .
$$

The density equations, Eqn. 1.7 and Eqn. 1.9, are used to derive an effective acceleration from Eqn. 1.6e,

$$
\begin{gathered}
\frac{d^{2} \chi}{d \xi^{2}}=\exp \chi-\frac{u_{0}}{\sqrt{u_{0}^{2}-2 \chi}} \equiv-\frac{\partial U}{\partial \chi}, \\
U(\chi)=1-\exp \chi-u_{0} \sqrt{u_{0}^{2}-2 \chi}+u_{0}^{2} .
\end{gathered}
$$

This equation is analogous to the acceleration of a pseudoparticle in an effective potential. In this representation, $\chi$ represents the coordinate of the pseudoparticle and $\xi$ corresponds to time. This allows the mechanical energy of the pseudoparticle to be written as an integral of motion: $W=\frac{1}{2}\left(\frac{d \chi}{d \xi}\right)^{2}+U(\chi)=$ const, where the constant is zero. Therefore, the integral of Eqn. 1.12a is rewritten as,

$$
\frac{1}{2}\left(\frac{d \chi}{d \xi}\right)^{2} \approx\left(\frac{1}{2}-\frac{1}{2 u}\right) \chi^{2}
$$

Eqn. 1.13 is the truncation to second order of the Taylor expansion of $U(\chi)$ in the limit of $\chi \rightarrow 0$. This limit corresponds to the pseudoparticle being at some 


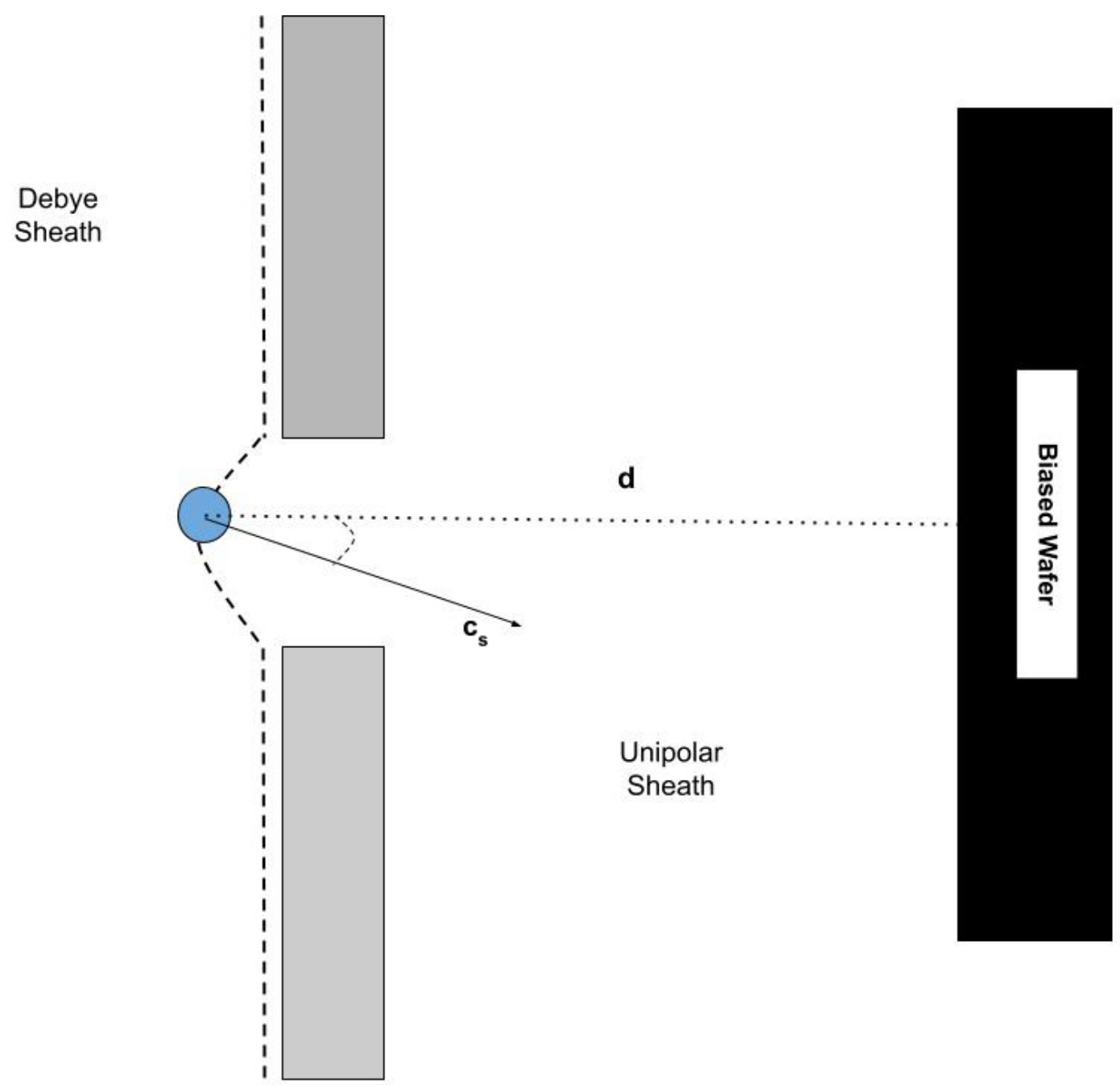

Figure 1.8: Diagram of the boundary between the Debye and unipolar sheaths. According to Eqn. 1.13 the ion will exit the Debye sheath normal to the boundary with a velocity equal to the ion sounds speed $c_{s}$. The ion will exit at some angle $\theta$ with respect to the beam normal and travel a distance $d$ through the unipolar sheath.

velocity $v_{0}$ due to zero potential, i.e., the entrance to the sheath. Since the left-hand side of Eqn. 1.13 is non-negative, a solution exists for positive values of the righthand side. Possible solutions are satisfied for $u^{2} \geq 1$, or in dimensional variables with $v \geq c_{s}$.

This constraint on the ion velocity as ions enter the sheath is known as the Bohm criterion [32]. Therefore, as an ion exits the sheath it will be traveling at the ion sound speed, normal to the sheath at the point of exit (Fig. 1.8). 


\subsubsection{Current Density}

The Bohm criterion is important for the extraction of ions because it sets up a minimum velocity at which ions leave the bulk plasma. Current density $j$ is the amount of charge that passes through an area in a given time and is defined as

$$
j=e n v .
$$

where $n$ and $v$ are the density and velocity respectively. In the case of ions falling through the sheath, their velocity is predicted to reach the Bohm velocity and therefore the theoretical minimum current density extracted due to a Debye sheath is $j_{s}=e n c_{s}$.

Once the ions fall through the Debye sheath they enter a second, unipolar sheath. This is a region that is devoid of electrons since they are repelled at the Debye sheath. The potential in this region is found using Eqn. 1.13 in the limit of $\chi \rightarrow+\infty$. In this limit, the pseudoparticle is on the other side of the Debye sheath, far from the boundary. This allows for Eqn. 1.13 to simplify to,

$$
\frac{1}{2}\left(\frac{\partial \chi}{\partial \xi}\right)^{2}=u \sqrt{-2 \chi}
$$

which has a solution of the form

$$
\chi=\left(\frac{u^{2}}{2}\right)^{\frac{1}{3}}\left(\frac{3 \xi}{2}\right)^{\frac{4}{3}}
$$

Returning to dimensional variables, Eqn. 1.16 far from the boundary yields an expression for $\phi$,

$$
\phi=-\frac{T_{e}}{e}\left(\frac{v_{\circ}^{2}}{2 c_{s}^{2}}\right)^{\frac{1}{3}}\left(\frac{3 z}{2 \lambda_{D}}\right)^{\frac{4}{3}} .
$$


Equation 1.17 rearranges to give,

$$
j_{3 / 2}=\frac{\sqrt{2}}{9 \pi \sqrt{m_{i}}} \frac{|\phi|^{\frac{3}{2}}}{z^{2}}
$$

which is the current density in a unipolar sheath. This current density gives an upper limit on the allowable current in the unipolar sheath for a given potential. The limitation is caused by space-charge effects in the region, as more ions are forced together, without electrons, they will repel and eventually cause beam divergence. To mitigate this effect, it is typical for ion extraction devices to introduce electrons into the beam to reduce the space-charge effects and increase the allowable extracted current. If the potential is supplied by an external source instead of the sheath, then Eqn. 1.18 takes on a slightly different form,

$$
j_{C L}=\frac{\sqrt{2}}{9 \pi \sqrt{m_{i}}} \frac{|U|^{\frac{3}{2}}}{d^{2}} .
$$

This is the well-known Child-Langmuir law [33] where $U$ is the externally applied potential and $d$ is the distance between the emitting surface and the ion collector.

Equation 1.14 gives the current density inside the plasma as the ions fall through the sheath while Eqn 1.19 gives the current density outside the plasma. The current density must be continuous at the boundary, $j_{s}=j_{C L}$, and equating them gives

$$
d=\frac{\sqrt{2}}{3}\left(\frac{2 e|U|}{T_{e}}\right)^{\frac{3}{4}} \lambda_{D},
$$

an expression for the length of the unipolar sheath.

\subsubsection{Plasma Meniscus}

The border of the Debye sheath and the unipolar sheath, referred to as the plasma meniscus, is an important boundary for controlling ion extraction. As ions move from the bulk of the plasma to the extraction region, they feel no extraction forces 


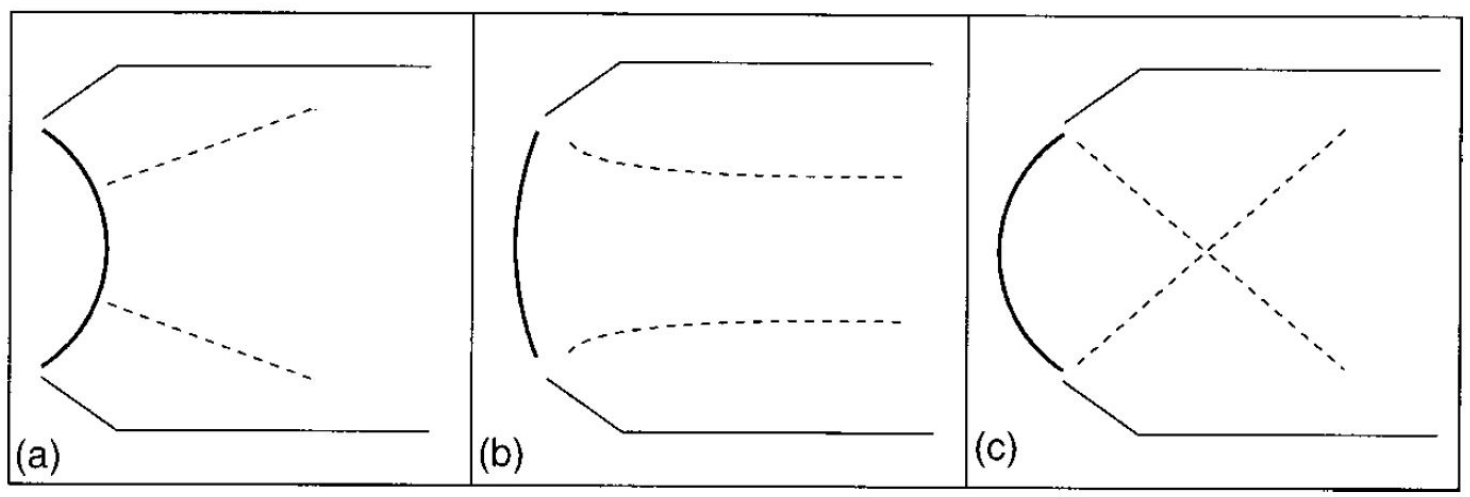

Figure 1.9: Beam focusing due to meniscus shape. a) Divergent beam from a convex shape, b) collimated beam from a flat boundary, and c) convergent beam from a concave shape. Reproduced with permission from Ref. [34], all rights reserved.

until they encounter the potential structures at the boundary. Then, as ions pass through the Debye sheath, they are accelerated to $c_{s}$ normal to the Debye sheath's surface. In the aperture region, the plasma meniscus boundary is dependent on the properties of the Debye and unipolar sheaths, giving a radius of curvature to the plasma meniscus, as shown in Fig. 1.9.

The radius of curvature creates a convex, concave, or parallel meniscus boundary based on the relative balance of forces from the extraction potential and the source [34]. In the source, an increase in ion population will reduce the Debye length of the sheath and force the plasma meniscus out through the aperture. Opposing this force is the electrostatic extraction potential that will push the unipolar sheath into the source. Since the boundary is created by the balancing of two forces, the source force and the electrostatic force, the meniscus is manipulated by changing system parameters. By increasing the ion number density, by increasing the input power, the plasma meniscus becomes more convex. If these parameters are lowered, or the applied potential is increased, then the meniscus will become more concave. If the two forces are perfectly balanced then the boundary becomes a flat surface spanning the aperture.

Since ions are accelerated by the sheath perpendicular to its equipotential lines, their trajectories are determined by the meniscus shape. In this way, the plasma 
meniscus is an electrostatic lens through which an ion beam's focal point is controlled. The velocity of an ion accelerated by an arbitrary potential is

$$
v=\sqrt{\frac{2 q V}{m_{i}}}
$$

Unless the boundary is planar, then ions will exit with some angular distribution of velocities. Once out of the Debye sheath the ions are then subject to the onaxis electric field from the biased wafer and Coulomb forces from nearby ions. The extraction potential will only change the parallel velocity while the ions will keep their transverse motion, determined from the meniscus's curvature. This creates an equation analogous to Snell's Law for optics:

$$
\begin{aligned}
v_{1} \sin \theta_{1} & =v_{2} \sin \theta_{2}, \\
\frac{v_{2}}{v_{1}} & =\frac{\sin \theta_{1}}{\sin \theta_{2}}=\sqrt{\frac{V_{2}}{V_{1}} .}
\end{aligned}
$$

Therefore, the plasma meniscus is thought of in terms of conventional optics such that a convex meniscus will produce a divergent beam, a flat meniscus will produce a collimated beam, and a concave meniscus will produce a convergent beam as shown in Fig. 1.9.

\subsection{Motivation}

The extraction region is complex and determines the extracted beam dynamics. Recent investigations into ion beams focus on the characterization of how controllable source parameters affect the beam [5]. In this way, the properties of an ion beam are tuned externally and without the need for significant system reconfiguring. However, to exert more control on the ion beam, the plasma meniscus needs to be more thoroughly understood. There are no direct measurements of the plasma meniscus, and most studies of the plasma meniscus focus on numerical simulations 
of the boundary. While these simulations help in understanding the focal point of extracted beams [35-37], they are based on the sheath assumptions in Section 1.3.1 and remain to be confirmed experimentally. Therefore, there is a need for experimental understanding of ion dynamics around the meniscus to confirm the sheath structure and its dependencies.
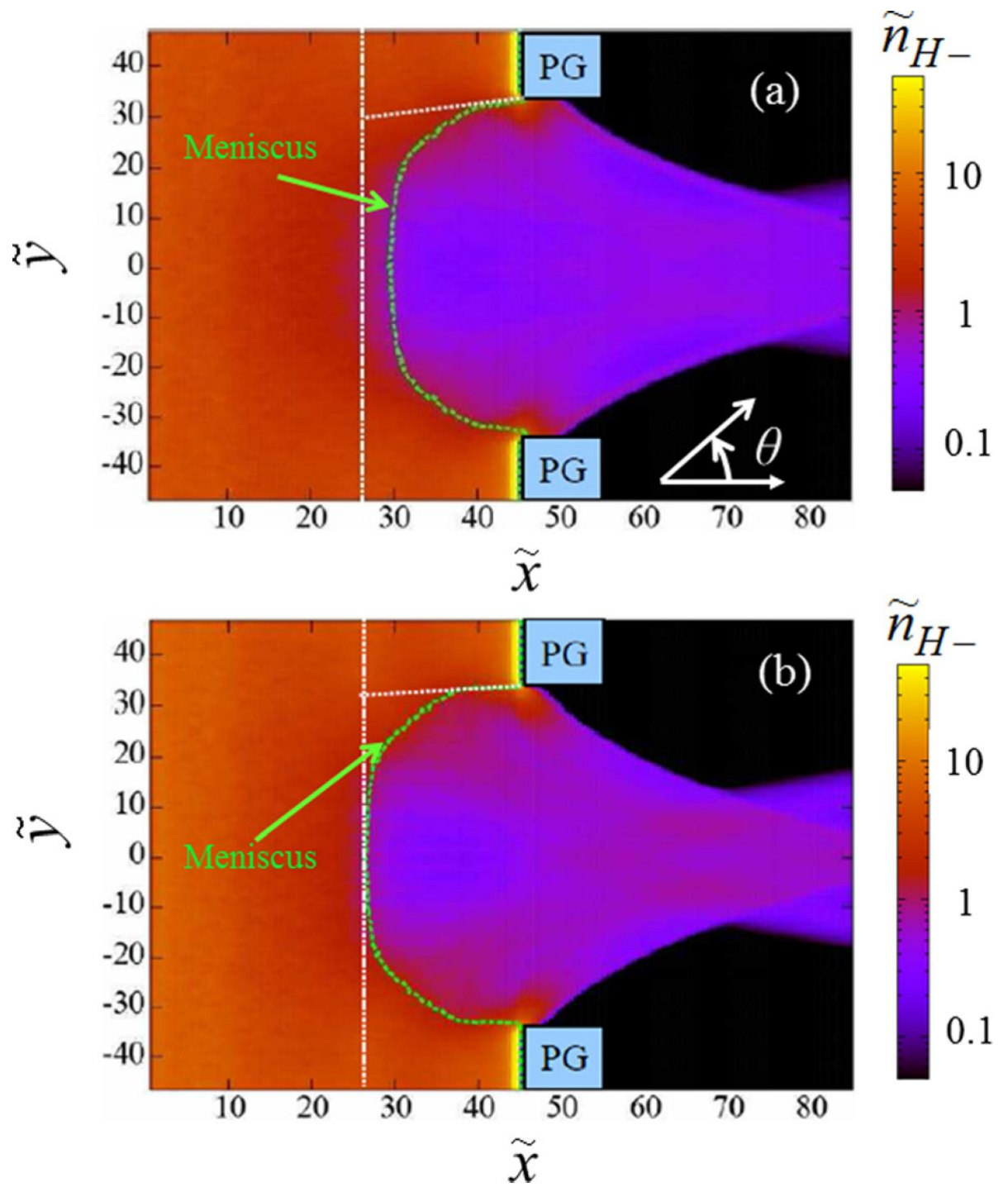

Figure 1.10: Simulations of an extracted $\mathrm{H}^{-}$plasma with a beam halo. a) and b) show how the meniscus depth can impact the focal length of the ion beam and beam halo. Reproduced with permission from Ref. [38], all rights reserved. 


\subsubsection{Neutral Beam Injectors}

Another active area of research that depends on meniscus modeling is neutral beam injection (NBI) systems. NBI is a method used in the plasma heating and current drive of magnetic confinement devices [39-41]. The International Thermonuclear Experimental Reactor (ITER) is expected to be heated by two NBI systems that will supply a total of $33 \mathrm{MW}$ of power over $3600 \mathrm{~s}$ [42]. NBI can deliver very hot neutrals into a plasma by first extracting a negative ion. This negative ion is pulled from a bulk plasma and then accelerated to high energies. The ion, $\mathrm{H}^{-}$in fusion applications, is then neutralized by an electron gas and injected into the plasma.

The extraction of negative ions is, at its core, the same process as positive ions. Therefore, when a positive potential is applied, a meniscus will form. This system follows the same principles of focusing and space-charge limitations, but with added complications. In these systems the extracted beam is surrounded by a secondary, over-focused beam [43]. This phenomenon is known as a beam halo. The beam halo is undesirable because it is poorly focused, decreases ion beam transport, and increases accelerator grid heat loads [44].

The beam halo is caused by surface emitted ions from the plasma grid [45]. These ions are produced near the plasma meniscus but with velocities opposite the primary beam as they fall through the sheath. This causes the unfavorable trajectories of the beam halo, shown in Fig. 1.10, where the ions are over-focused in the source and divergent outside. This is still an open problem for NBIs as confirmation and reduction of this beam halo effect is needed to improve heating of fusion devices. Therefore, understanding the plasma meniscus is crucial to accurately modeling negative ion sources [38]. 


\subsubsection{D Architecture}

Semiconductors continue to progress further towards high-aspect ratios through nano-manufacturing to meet the demands of logic and memory devices. As features on ICs get smaller, they will eventually start reaching the atomic scale, with a dozen or less silicon atoms constituting the feature [46]. While this may be achievable, such features would be very susceptible to imperfections and the cost of processing may make such semiconductors prohibitively expensive. Atomic layer etching shows that sub-nanometer, uniform lateral etching is possible but with lower throughput [47]. While this technique will play a role in the future of two-dimensional transistors, it is also the absolute lower limit of what is achievable for processing in two-dimensions.

It is therefore necessary to transition from two-dimensional transistors to threedimensional transistors. Multi-stack arrays are a technology currently used, but these are two-dimensional semiconductors stacked and connected to form a three-

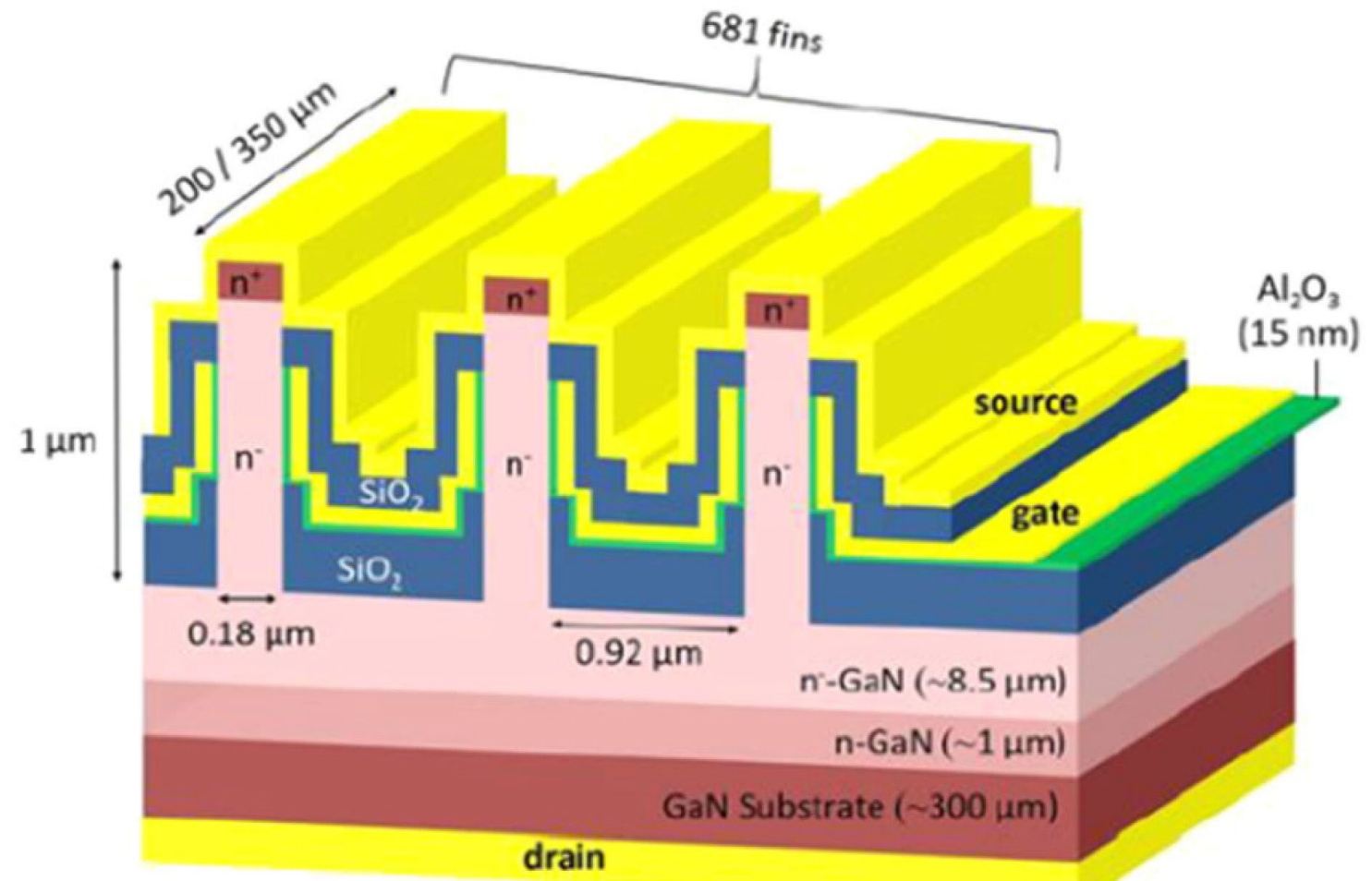

Figure 1.11: Schematic of a 3D, GaN Fin-FET gate. Reproduced from Ref. [48] under the terms of Creative Commons CC-BY. 
dimensional structure [49]. Figure 1.11 shows what a truly 3D transistor looks like [48]. In this type of device, the features are all grown on one another so that the full volume of the structure is utilized. Additionally, creating features in threedimensions relaxes some of the minimum spatial requirement for chips with equal processing power.

In two-dimensional architecture, depending on the feature being created, there is typically a dimension that is not important for the performance of the semiconductor. This is not the case with three-dimensions where anisotropy is paramount for controlled processing. Therefore, the extracted ion beam needs to reliably target sidewalls with a constant beam angle. If a physical blocker is placed in the extraction region of the beam, near the meniscus, then the resulting ion beam will have only a small subset of the full beam's angular distribution (Fig. 1.12). The mean angular distribution produced this way is then dependent on the radius of curvature of the plasma meniscus, allowing for real-time beam steering through manipulation of source parameters.

Therefore, to actualize three-dimensional transistors for production, highly controllable ion beams are needed. As shown in Fig. 1.11, not only do layers need to be thin, but ions need to be able to uniformly access the trench walls in between fins. Tight tolerances and a need for $\sim 10 \mathrm{~nm}$ features are already approaching the limitations of current ion sources [50]. Additionally, high-energy ion beams potentially cause lattice damage from the energetic bombardment of ions [8] or electrical damage where gate oxides break down due to uneven charge buildup from non-uniform plasma [51].

To inform the next generation of plasma processing sources, the plasma meniscus needs to be well understood. Currently, the extraction optics used in industry have apertures with dimensions larger than the Debye sheath, making meniscus formation unlikely. At smaller aperture sizes, where a meniscus is formed, this boundary determines properties of the extracted beam such as the current density and focal 


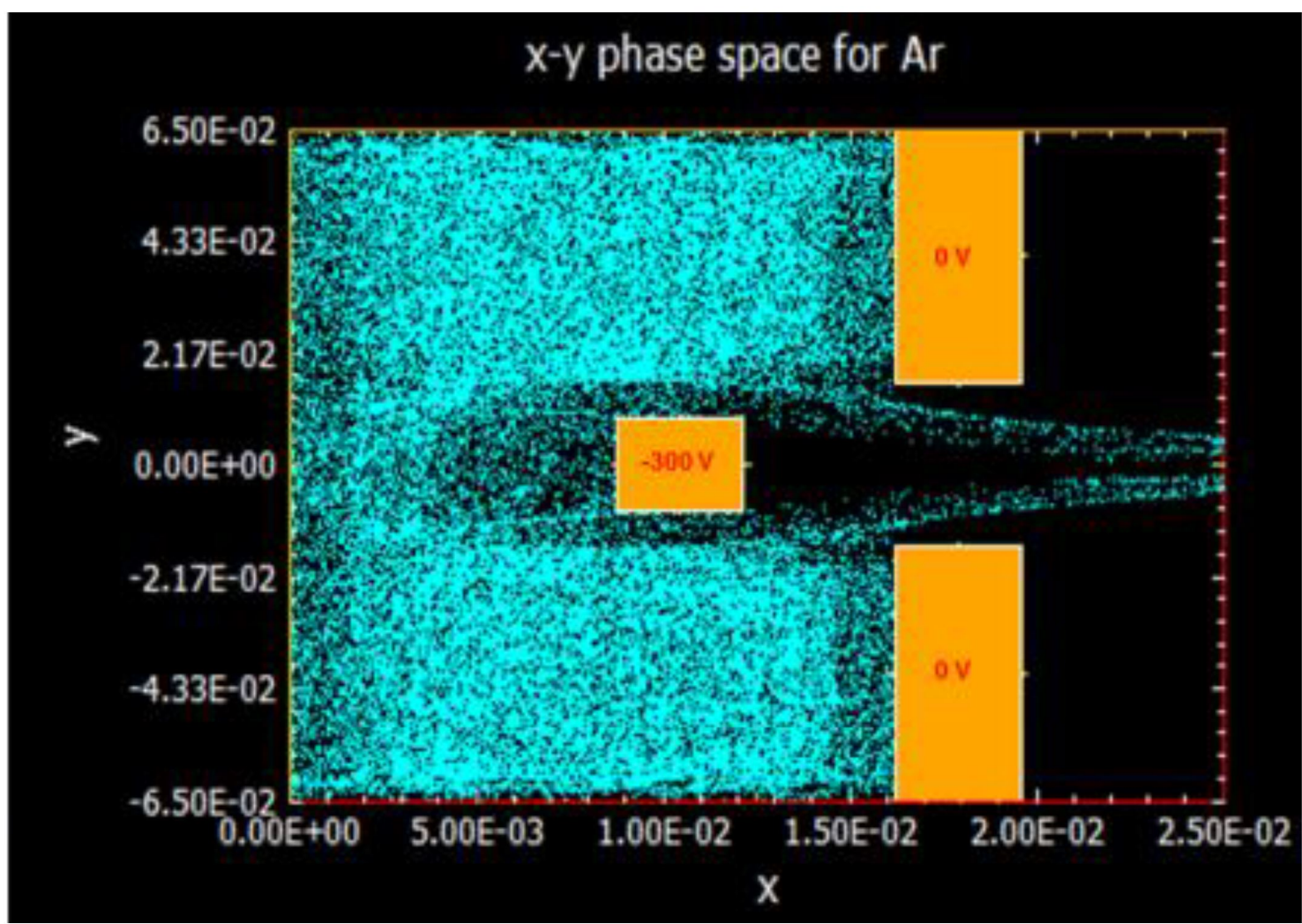

Figure 1.12: An Object-Oriented Particle-in-Cell (OOPIC) model of ion extraction with a physical blocker. In this model, the blocker also supplies the bias potential to form a controllable plasma meniscus. The resulting ion "beamlets" have a smaller angular distribution than a full beam.

spot. Therefore, the meniscus determines both the throughput for etching and doping, as well as the minimum feature size able to be processed by the beam.

The current need in industry is for a low energy, high density ion source with precise control over the extracted beam. A wide processing planar beam, however, does not have high enough spatial control for non-planar devices, like three-dimensional structures [15]. Instead, a small extraction aperture with a meniscus presents a possible solution, exerting a greater level of control in the extraction region. Thus, it imperative to understand the processes of ion extraction from the plasma meniscus and how source parameters affect this boundary. With this goal in mind, the work presented here focuses on the ion properties across the plasma meniscus. 


\section{Chapter 2}

\section{Experimental}

\subsection{Inductively Coupled Plasmas}

Hittorf first discovered ICPs in 1884 when he reported an "electrodeless ring discharge" [52]. This was achieved by wrapping an evacuated chamber in copper wire connected to a discharging Leyden jar. He hypothesized that the plasma was generated by inductive forces. However, this generated debate as many of his peers believed that the plasma was generated by the high and low voltage ends of the coil, acting as a capacitor and coupling to the plasma similar to an radio-frequency (rf) diode [53]. This discussion lasted nearly fifty years until both sides, surprisingly, were proven correct.

There are two distinct operating modes in ICPs. In an electrostatically dominated power coupling mode, or E-mode, the plasma is sustained primarily by capacitive coupling from the high voltage antenna. In an electromagnetically dominated power coupling mode, H-mode, plasmas are produced mainly due to the inductive field created by the rapidly changing current in the antenna. These coupling methods exist simultaneously in ICPs; the distinction comes from which method is transferring most of the power to the plasma. These different coupling modes differ 


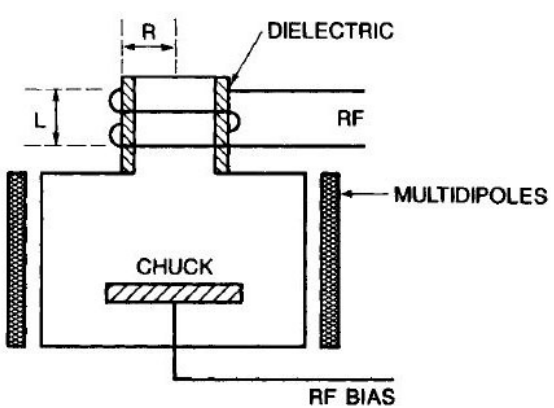

(a)

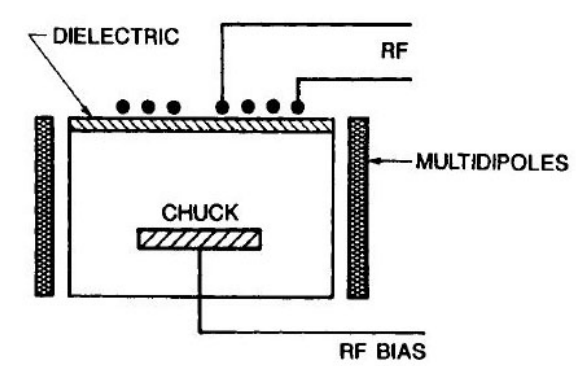

(b)

Figure 2.1: The two types of antenna designs used for ICP generation are a) the spiral antenna and b) the planar antenna. Reproduced with permission from [1], all rights reserved.

in that H-mode plasmas have much higher densities, lower plasma potential, and are visually much more luminous than E-mode plasmas $[1,54,55]$.

\section{$2.2 \quad$ Antenna}

ICPs are driven by spiral antennas, typically in the megahertz range. There are two main configurations of the antenna; a cylindrical antenna winding that acts as a solenoid with the core being the plasma vessel, and a planar antenna, described as a "flat helix" or a "stove-top coil" that sits at the plasma boundary. The two types of antenna are shown in Figure 2.1.

In order to produce an induced field, the antenna typically has a spiral shape to it. This is because ICPs need the curl of $\mathbf{E}$ to be non-zero such that

$$
\nabla \times \mathbf{E}=-\frac{\partial \mathbf{B}}{\partial t}
$$

where $\mathbf{E}$ is the electric field vector, $\mathbf{B}$ is the magnetic field vector, and $t$ is time. Therefore, for the magnetic induction field to be created, there must be a non-zero curl of the electric field produced from the antenna. Since current supplied to the antenna is time varying, it will create an azimuthal electric field, replicating the 


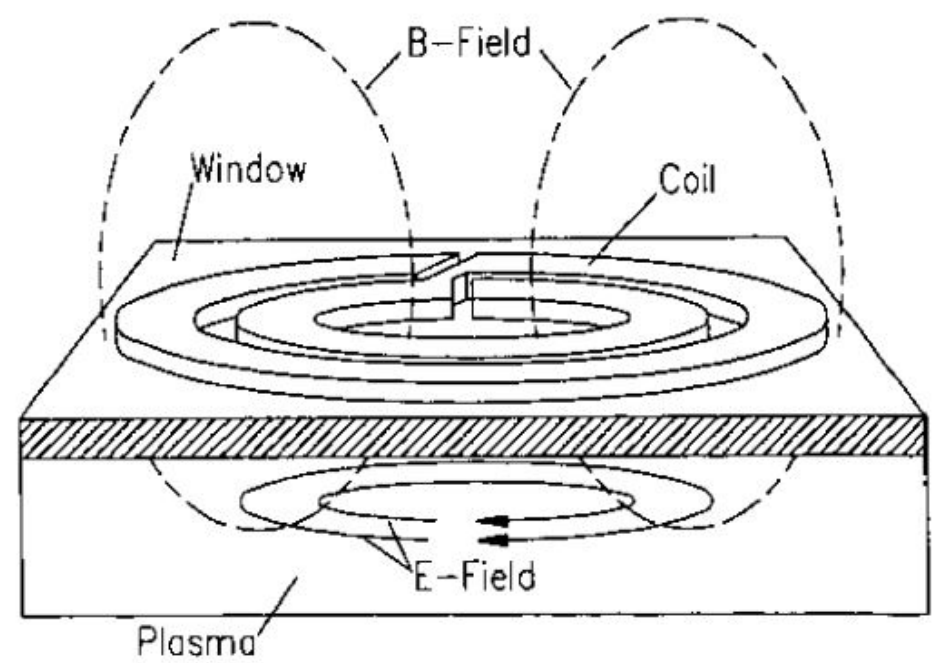

Figure 2.2: Schematic of the induction fields due to a spiral antenna. The time varying $\mathbf{B}$-field generated by the antenna passes through a dielectric barrier and creates an E-field inside the source. Reproduced with permission from [56], all rights reserved.

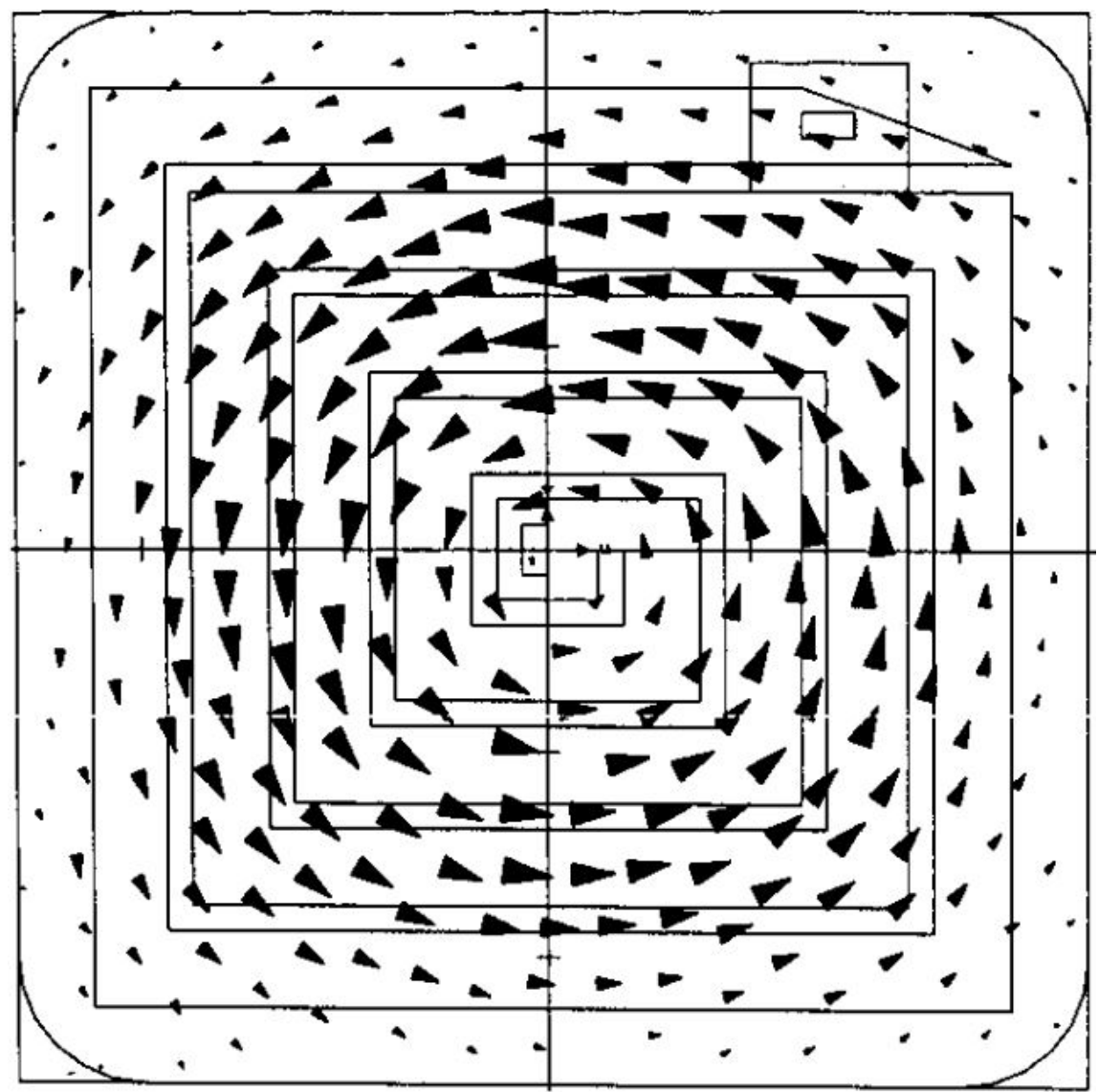

Figure 2.3: Simulated vector field of the induced E-field in an ICP. The solid lines represent the superimposed spiral antenna. Reproduced with permission from [56], all rights reserved.

structure of the antenna inside the plasma chamber. Figure 2.2 shows a schematic 
of the induced fields in a planar ICP source and Fig. 2.3 shows the resulting induced E-field inside the plasma chamber.

\subsubsection{Transferred Power}

Electromagnetic theory of spiral antennas is well documented in the literature [5760]. The treatment of planar coils is much more complex due to finite geometry effects [53]. A derivation of planar theory is given in Reference [61]. However, it is common to avoid these complexities by representing the antenna-plasma system as a transformer circuit $[62,63]$. The antenna is considered the primary winding while the plasma is modeled as a single turn, secondary winding. This replaces the complex theory of planar coils with a simplified model that shows the impact of individual circuit components on plasma generation in an ICP.

Using standard transformer equations, the power transferred by the circuit to the plasma is calculated. The reactance in the primary winding of a transformer is given by,

$$
Z_{\text {coil }}=\frac{\omega M^{2} R_{p l}}{R_{p l}^{2}+\omega_{t r a n}^{2} L_{p l}^{2}}+i\left(\omega_{t r a n} L_{c o i l}-\frac{\omega_{t r a n}^{3} M^{2} L_{p l}}{R_{p l}^{2}+\omega_{t r a n}^{2} L_{p l}^{2}}\right)
$$

where $\omega_{\text {tran }}$ is the driving frequency in $\mathrm{rad} / \mathrm{s}, L_{\text {coil }}$ and $L_{p l}$ are the inductances of the coil and plasma, respectively, $R_{p l}$ is the resistance of the plasma, and $M$ is the mutual inductance. The induced power produced by a primary winding is calculated by taking the real part of the reactance,

$$
\begin{aligned}
P_{\text {ind }} & =I^{2} \operatorname{Re}\left(Z_{\text {coil }}\right) \\
& =C \sqrt{I^{2}-I_{\text {threshold }}^{2}}
\end{aligned}
$$


where $I$ is the current in the antenna, $C$ and $I_{\text {threshold }}$ are constants given by

$$
\begin{array}{r}
C=k \sqrt{R_{p} P_{\text {ind }}} \sqrt{\frac{L_{\text {coil }}}{L_{p l}}} \\
I_{\text {threshold }}=\frac{\sqrt{R_{p l} P_{\text {ind }}}}{\omega_{\text {tran }} k \sqrt{L_{\text {coil }} L_{p l}}},
\end{array}
$$

and $k$ is the mutual inductance for transformers,

$$
k=\frac{M}{\sqrt{L_{c o i l} L_{p l}}} .
$$

Expressing Eqn. 2.3 in terms of $C$ and $I_{\text {threshold }}$ is desirable since these quantities are constants. Electron-neutral collisions are responsible for dissipating power in an ICP. Therefore, the plasma resistance is inversely proportional to plasma density [1]. Furthermore, plasma density increases linearly with power in an ICP $[54,56]$. Thus $R_{p l} P_{\text {ind }}$ is constant in Eqn. 2.5. Assuming $M$ remains constant, then Eqn. 2.5 gives a value based on plasma properties that defines the minimum antenna current necessary for the onset of H-mode.

\subsubsection{Dissipated Power}

For a single electron in a uniform oscillating electric field, the time averaged energy transferred from the field to the electron is zero. For an ensemble of electrons, however, there are electron-electron and electron-neutral collisions that break down the coherent motion over a given time period. This gives rise to a net transfer of power into the plasma. For a large enough volume of electrons, it is appropriate to use the macroscopic fluid description for the plasma. This description introduces a plasma conductivity term $\sigma_{p}$, which is responsible for dissipating energy through Ohmic heating. The power dissipated by this heating is given by 


$$
\begin{aligned}
P_{\text {Ohmic }} & =\frac{1}{2} \operatorname{Re}\left(\mathbf{J} \cdot \mathbf{E}^{*}\right) \\
& =\frac{1}{2}|\mathbf{J}|^{2} \operatorname{Re}\left(\sigma_{p}^{-1}\right) .
\end{aligned}
$$

However, this type of heating is only effective when the electron-neutral and electronelectron collision frequency are much larger than the EM wave's frequency. Ohmic heating is present in both E-mode and H-mode discharges.

A second mechanism for heating in an ICP is known as the anomalous skin effect. This is a "warm plasma" effect that causes collisionless heating and is only present in H-mode discharges. To describe this effect, consider a collection of electrons with some thermal motion. These electrons will constantly sample the induced electric field at different locations. Locally the field will average to zero over an rf period and no energy is gained by the electrons. If an electron has sufficient energy, it can travel through a region of high field strength and exit before a full rf period is complete, thus gaining energy. If the condition of

$$
\omega \delta \lesssim\left(\frac{k_{B} T_{e}}{m_{e}}\right)
$$

is met then the electron can gain energy from the field, where $\omega$ is the frequency of the antenna and $\delta$ is the skin depth, defined as

$$
\delta^{-1}=\operatorname{Im}\left(\frac{\omega}{c}\left[1-\frac{\omega_{p e}^{2}}{\omega\left(\omega-i \nu_{e n}\right)}\right]^{1 / 2}\right)
$$

Here, $\omega_{p e}=n e^{2} / \epsilon_{\circ} m_{e}$ is the plasma electron frequency, $c$ is the speed of light in vacuum, and $\nu_{e n}$ is the electron-neutral collision frequency.

The skin depth is the distance a particular EM wave travels before its amplitude is attenuated by $1 / e . \delta$ is the typical distance electrons need to travel in order to sample different field strengths. Therefore, the anomalous skin effect is limited to a range of operating frequencies. If an rf period is too short, the electrons will not have time to transverse a skin depth. Additionally, if the frequency is excessively low, the 
skin depth is larger than the plasma chamber. Therefore, the frequency must satisfy Eqn. 2.8 and $(\delta \lesssim L)$ for an electron to gain energy from the induced field [64]. In what can only be describes as a "fortuitous coincidence" [64], the frequencies required for effective collisionless heating are near the conventional $13.56 \mathrm{MHz}$ [65].

The last contribution to dissipation of antenna power comes from stochastic, collisionless interactions. This heating mechanism is predominately in E-mode discharges and arises from the potential variation in time varying fields. The antenna potential will form a sheath on the dielectric barrier which oscillates into and out of the bulk of the plasma over the course of an rf period. As electrons enter the sheath near the dielectric barrier, they change velocity and bounce off the sheath edge. Depending on the sheath's oscillation when the electrons are repelled by the sheath edge, they can either gain or lose energy from the sheath. This heating is stochastic due to the randomness of electrons colliding with the sheath [66].

\subsection{Coupling Modes}

The onset of H-mode operation is typically defined by the minimum current needed to make a skin depth that is approximately equal to the discharge length [67]. However, this is too strict a definition as slight fluctuations in the potential of induced fields and sheaths result in significant changes in the percent of ionization. Therefore, it is a more robust method to define this transition point in terms of the pressure in the discharge, which is proportional to rf power [68].

Skin depth is related to the wavenumber of the EM waves by

$$
k=\frac{\omega}{c} \operatorname{Im}\left(\kappa_{p}^{1 / 2}\right)=-\delta^{-1}
$$

where $\kappa_{p}=1-\frac{\omega_{p e}^{2}}{\omega\left(\omega-i \nu_{e n}\right)}$ is the plasma dielectric constant. From this description, Eqn. 2.9 has two limiting cases, a high-pressure regime and a low-pressure regime. 
The distinction between the two is dependent on the electron-neutral collision rate $\nu_{e n}$ relative to the driving frequency $\omega$. In the low-pressure regime $\left(\nu_{e n} \ll \omega\right)$, Eqn. 2.9 becomes $[1]$

$$
\delta_{\text {low }}=\left(\frac{m_{e}}{e^{2} \mu_{\circ} n_{e}}\right)^{1 / 2} .
$$

$\mu_{\circ}$ is the permeability of free space, and $n_{e}$ is density of the electrons. If $\nu_{e n} \gg \omega$ then the discharge is considered to be in a high-pressure regime and the skin depth is given by

$$
\delta_{h i g h}=\left(\frac{2 m_{e} \nu_{e n}}{\omega \mu_{0} e^{2} n_{e}}\right)^{1 / 2} .
$$

In either of these pressure regimes, ICPs will operate in E-mode or H-mode for low and high electron densities, respectively. If the skin depth is less then the dimensions of the ion source chamber, then the discharge is considered high density (H-mode), while if the skin depth is on the order of or larger than the source dimensions the discharge is low density (E-mode) [1].

\subsubsection{Low Pressure}

The E-mode is the dominant coupling mode of ICPs at low pressures. While there is an induced electric field present, even during E-mode, there is not enough electron density in the plasma for its contributions to overtake the capacitive effects of E-mode heating mechanisms. Therefore, the plasma is heated similarly to a DC discharge plasma [69]. Some fraction of the antenna voltage is able to pass through a dielectric window on the ICP source and create a sheath on the other side of the dielectric. This sheath provides the electric potential needed to accelerate electrons, ionize the neutral gas, and heat the plasma. 
The wavenumber for EM waves in a low-pressure case $k_{d}$ is approximated by

$$
k_{d} \approx \pm \frac{\omega}{c} \sqrt{1-\frac{\omega_{p e}^{2}}{\omega^{2}}}
$$

Eqn. 2.13 presents two solutions depending on whether the wavenumber is real or imaginary. For high frequency EM waves $\left(\omega>\omega_{p e}\right)$, the waves are able to propagate in the plasma. At low frequency $\left(\omega<\omega_{p e}\right)$, the wavenumber is imaginary, which causes exponential decay of the wave. The border between propagating and decaying waves is the cutoff frequency, $\omega_{c o}=\omega_{p e}$. As mentioned, transferred power is related to electron density in ICPs. So density will increase as the antenna power increases, causing electrons to more efficiently absorb the EM wave, up to a certain density.

Equating the wavenumber from Eqn. 2.13 and the skin depth from Eqn. 2.11 gives a cutoff density of

$$
n_{d, c o}=\frac{m \epsilon_{0} \omega^{2}}{e^{2}}
$$

As the power to the antenna increases, and therefore the electron density increases, the effective power transferred to the plasma will increase up to a density limit of $n_{d, c o}[70]$. However, if electron density continues to increase, the dielectric sheath's thickness decreases. At sufficiently high densities, only a fraction of the antenna potential appears across the sheath, reducing plasma heating. Therefore, even though the density is increasing, the waves are not as effective at heating the electrons by stochastic and Ohmic mechanisms, resulting in a decrease of transferred power to the plasma. However, as density continues to increase past this cutoff density, the discharge is still maintained due to the onset of heating from inductive forces. 


\subsubsection{High Pressure}

H-mode discharges occur at sufficiently high pressures where there are enough electrons to respond to the induced electric field. This will accelerate electrons and collisionally heat the plasma to some degree, but the majority of this heating comes from the anomalous skin effect [71]. For a sufficiently large electron density, the skin depth is less than the dimensions of the plasma source, and ICP operates in the high density regime. At high pressures, Eqn. 2.10 becomes

$$
k_{D} \approx \pm \frac{\omega}{c} \sqrt{1-i \frac{\omega_{p e}^{2}}{\omega \nu_{e n}}} .
$$

Similar to the low-pressure scenario, the rf induction wave will propagate or attenuate depending on the value of the argument under the square root. Calculating Eqn. 2.15 in the limit of $\omega_{p e}^{2} \ll \omega \nu_{e n}$ simplifies to

$$
k_{D} \approx \pm \frac{\omega}{c}
$$

The cutoff density of electrons in this case is found by equating wavenumber and skin depth (Eqn. 2.16 and Eqn. 2.12), giving

$$
n_{D, c o} \ll \frac{m \epsilon_{0} \omega^{2}}{e^{2}}\left(\frac{2 \nu_{e n}}{\omega}\right) .
$$

Since $\nu_{e n}$ is proportional to density, the cutoff density is given as an inequality. This is the same form for the low pressure cutoff density (Eqn. 2.14) but with a new collisional term $\left(2 \nu_{e n} / \omega\right)$.

In the other limit, where $\omega_{p e}^{2} \gg \omega \nu_{e n}$, Eqn. 2.16 and Eqn. 2.12 become

$$
k_{D} \approx \pm \frac{\omega_{p e}^{2}}{c} \sqrt{\frac{\omega}{2 \nu_{e n}}}(-1+i)
$$




$$
n_{D, c o} \gg \frac{m \epsilon_{0} \omega^{2}}{e^{2}}\left(\frac{\nu_{e n}}{\omega}\right)
$$

respectively. By Eqn. 2.17 and Eqn. 2.19, a reasonable approximation for the cutoff density is given by $n_{D, c o} \approx m \epsilon_{0} \omega \nu_{e n} / e^{2}$. This shows that, like the low-pressure case, EM waves will continue to heat the plasma until some critical density, at which point the waves can no longer effectively transfer power to the electrons. Increasing electron density causes the skin depth to decrease to the point where the induced field cannot propagate throughout the discharge. Eventually, the skin depth will become small enough that the rf induction wave attenuates too quickly and cannot penetrate deep enough into the source. The causes majority the heating to occur near the antenna until the discharge can no longer be supported. This highlights one of the major constraints of ICPs; that they have a maximum density. Most ICPs can effectively power couple up to a density of $\sim 10^{-16} \mathrm{~m}^{-3}$, after which trying to increase density will block EM waves and reduce the transferred power sustaining the discharge [68].

\subsubsection{E \& H Mode Transitions}

The mechanism responsible for the $\mathrm{E}$ to $\mathrm{H}$ transition is twofold. First, a reduction in the dielectric sheath limits the capacitive contribution to plasma heating. Second, the increase in electron density increases the effectiveness of inductive heating. Now, there are enough electrons to effectively respond to the induced field. There will still be some contribution from capacitive effects, but at high densities, the anomalous skin effect is the dominant heating factor. This is shown in Fig. 2.4, where the E-mode (Capacitive coupling) and H-mode (Inductive coupling) contributions are shown over a range of electron densities. The solid lines represent the total power transferred from the antenna. The dashed and dotted lines show the individual contribution from inductive and capacitive coupling modes, respectively. The cutoff densities for $\mathrm{E}$ and H-mode operations are given by the local maxima of the peaks, 


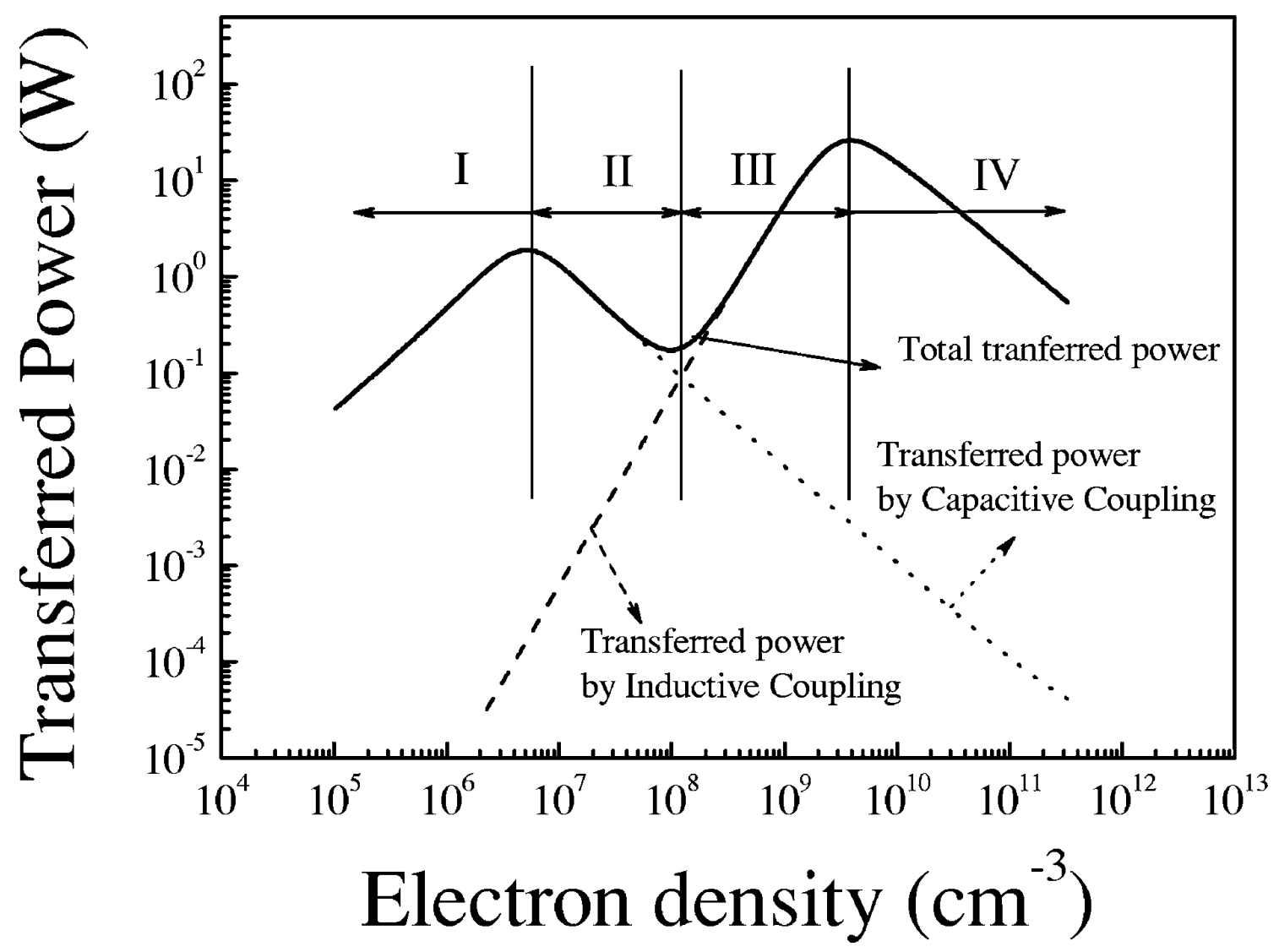

Figure 2.4: The total power transferred versus electron density for both capcitive and inductive coupling. In region I, the transferred power increases with density because the cutoff density has not been reached. Region II is after the cutoff density calculated for the low-pressure case $n_{d, c o}$. In region III, the contribution from the capacitive effects continue to decrease, but now the plasma is in H-mode and inductive effects are the predominant heating mechanism. Region IV is after the high-pressure cutoff density $n_{D, c o}$, and the transferred power will continue to decrease until the discharge can no longer be maintained. Reproduced with permission from Ref. [68], all rights reserved.

while the transition density for $\mathrm{E}-\mathrm{H}$ mode is the local minimum between the two peaks.

ICPs also exhibit hysteresis in the power necessary for $\mathrm{E}$ to $\mathrm{H}$ and $\mathrm{H}$ to $\mathrm{E}$ transitions. This hysteresis is as large as a factor of three between the transition power and minimum power necessary to maintain H-mode [61]. Hysteresis arises in ICPs due to non-linearities in the absorbed and dissipated power. There are many parameters which contribute to this phenomenon, such as multistep ionization and electronelectron collisions, but it is difficult to quantify each parameter's contributions [73]. An example of this hysteresis is shown in Fig. 2.5 where the transitions are apparent 


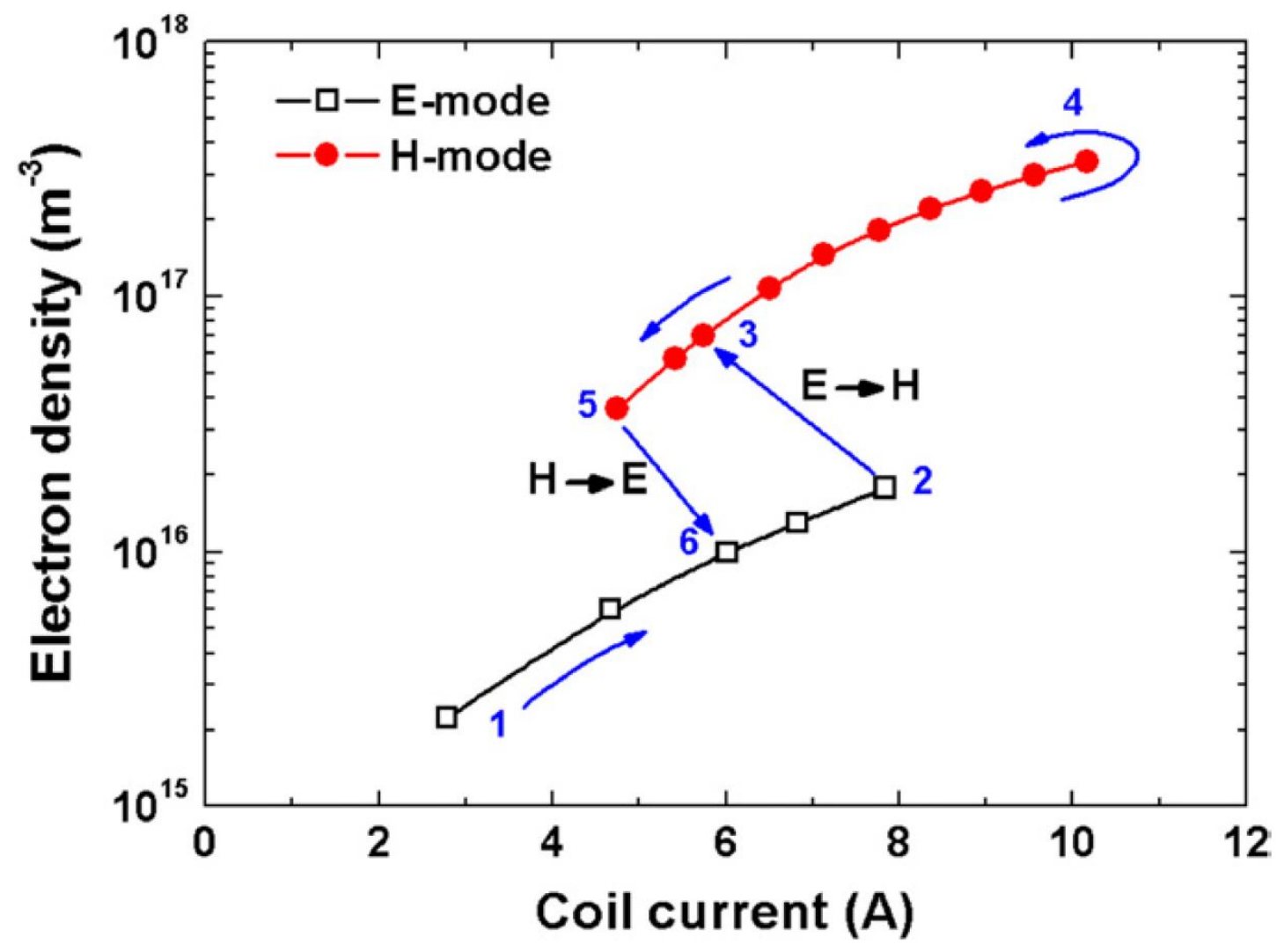

Figure 2.5: The current dependent hysteresis for E-H and H-E mode transitions. These two transitions occur at different antenna currents. Therefore, once H-mode is achieved, the plasma will remain in an inductive coupling even if the density drops. Reproduced with permission from [72], all rights reserved.

at different coil currents. For the remainder of this work, when referring to ICPs, they are assumed to be in H-mode unless otherwise stated.

\subsection{REVAN Chamber}

The experiments described here were performed in the Ribbon Experiment for ion Velocity and Angular distributioN (REVAN), shown in Fig. 2.6, at West Virginia University. The vacuum chamber is composed of a $36.25 " \times 16 " \times 15.5$ " tub with 0.25" thick walls of 6061 aluminum from Midwest Steel Supply, Inc., and was fabricated in-house. The chamber was designed to provide ample optical access of the source by three $20 " \times 4$ " ports on the top, bottom, and front of the chamber. These ports are covered by $21.5 " \times 5.5 " \times 0.5 "$ tempered glass from Mountaineer Glass 
\& Mirror LLC. Additionally, there are four 2.75" ports located on both the top and bottom of the chamber. The chamber also has two 8" ports on either side. One of these ports provides optical access while the other is connected to the vacuum system.

\subsubsection{Rf Circuit}

The ICP discharge studied here is generated by a Dressler Caeser RF Power Generator. This rf source produces $\leq 5 \mathrm{~kW}$ of power at $13.56 \mathrm{MHz}$. Despite being water cooled, excessive operating temperatures occurred at powers greater than 3 $\mathrm{kW}$, limiting the output power to $4 \mathrm{~kW}$. At $4 \mathrm{~kW}$ the source could operate for $\sim 12$

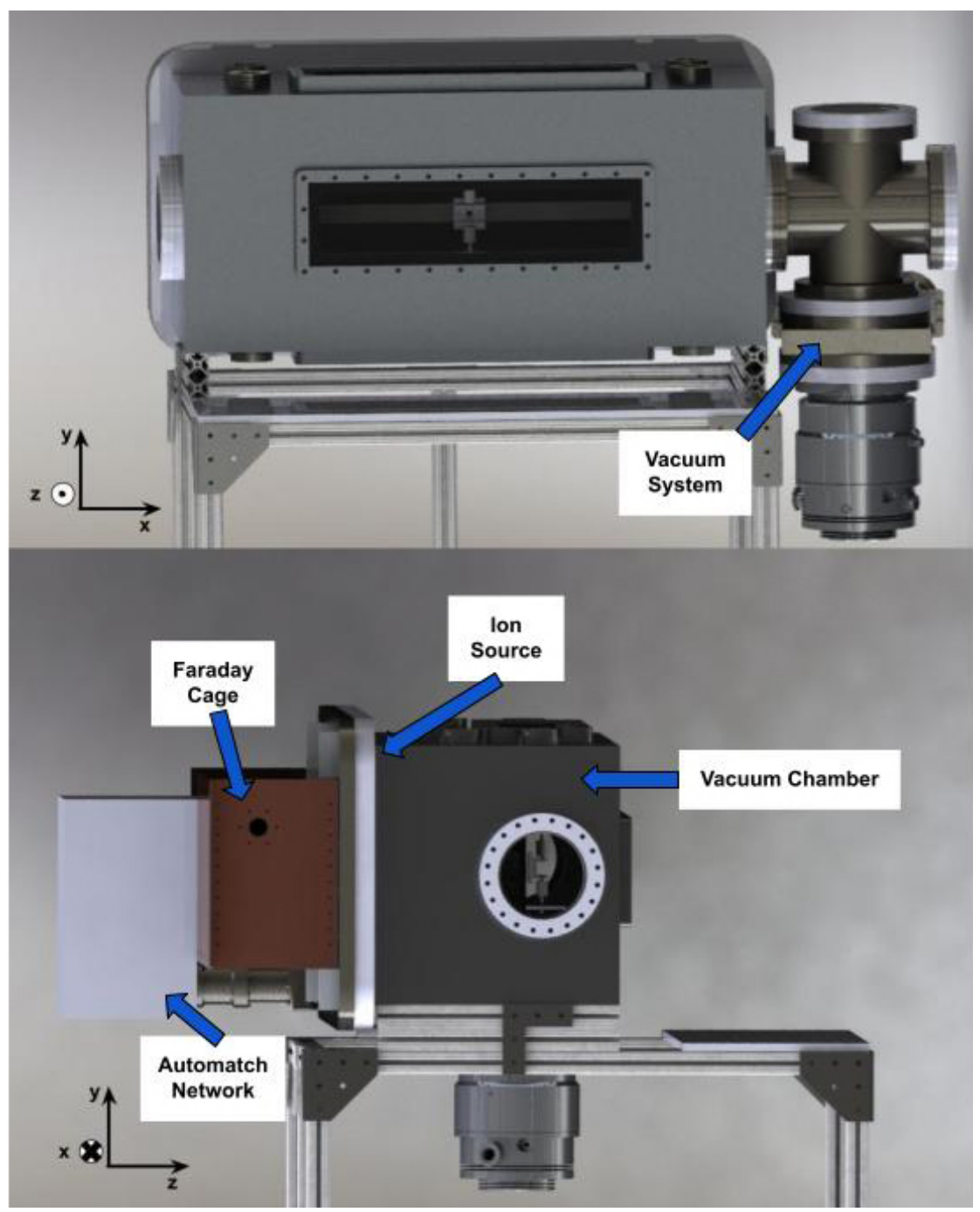

Figure 2.6: Renderings of the REVAN chamber. 
mins before it tripped an internal interlock. This made power cycling necessary for data acquisition at this rf power, resulting in a $\sim 25 \%$ duty cycle. Above $4 \mathrm{~kW}$, the source would overheat too quickly to obtain reliable data.

The generator was connected to an ENI MWH 100-01 impedance matching network designed for operation at $13.56 \mathrm{MHz}$. This is an auto-matching network that constantly tunes itself to minimize circuit impedance, thus maximizing coupled power to the plasma. It is a low-loss, L configuration circuit consisting of two vacuum-variable capacitors, which are controlled by precision stepper motors and a fixed inductor [74]. The two capacitors are in parallel with each other: the load capacitor $\mathrm{C}_{L}$, which is connected to ground, and the tuning capacitor $\mathrm{C}_{T}$, which is in-line with the antenna. The output of the auto-match network is connected to the antenna by $203 \mathrm{~mm}$ of stranded 4/0 welding cable. Figure 2.7 shows a circuit diagram for this system which is modeled according to the transformer description.

The antenna is a water-cooled, three turn, $\varnothing 0.5 "$ copper planar coil with an inductance of $50 \mu \mathrm{H}$. The antenna is roughly rectangular in shape with an area of $3.35 " \times 21.56 "$. This type of antenna excels at creating a large, uniformly heated

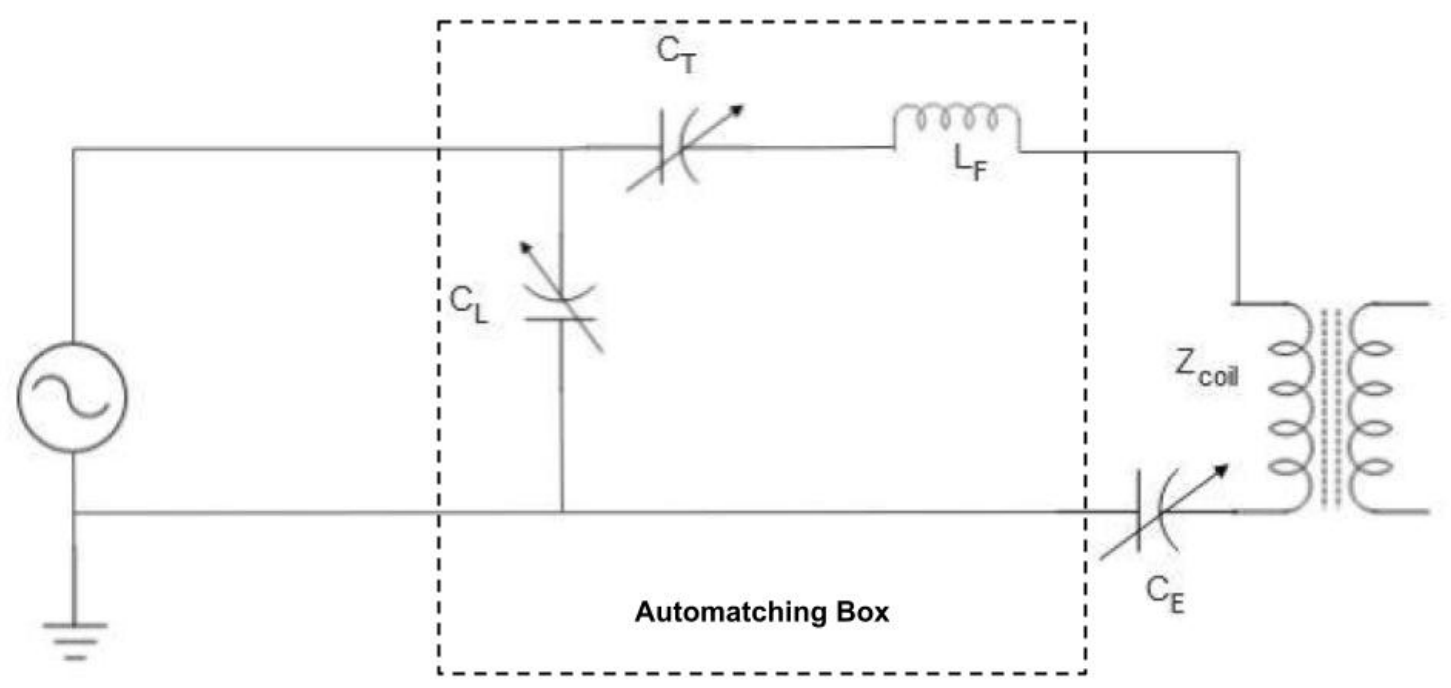

Figure 2.7: The circuit diagram connecting the rf generator to the antenna. The region inside the dashed box is the matching network. The circuit consists of a load capacitor $\mathrm{C}_{\mathrm{L}}$, a tuning capacitor $\mathrm{C}_{\mathrm{T}}$, a grounding capacitor $\mathrm{C}_{\mathrm{E}}$ and a fixed inductor $\mathrm{L}_{\mathrm{F}}$ 
plasma volume [56]. One leg of the antenna is connected to a $5-170 \mathrm{pF}$ Comet Variable Vacuum Capacitor by another length of $152 \mathrm{~mm}, 4 / 0$ welding cable. The grounding capacitor is mounted to REVAN's Faraday shield, which grounds itself through the rf generator. This capacitor is externally tuned to either set the initial condition for the matching network or to change the impedance of the circuit during operation in order to ensure favorable, low impedance matching. The antenna and welding wire are contained in a 1/8" thick copper Faraday cage to block leakage of the rf fields.

\subsubsection{Source}

For this work, Applied Materials Inc. supplied a proprietary ion source. It has two main stages; the first is exposed to atmosphere, separated from vacuum by a dielectric window, made of 6061-T6 aluminum, and is water cooled by an external chiller. This stage houses the antenna coil. The second stage is the ion source and is separated from atmosphere by a 1" thick, quartz dielectric barrier. This barrier covers an area of $24 " \times 5 "$ and was procured from Hayward Quartz Technology Inc. The ion source also has a magnetic cusp field, produced by eight, $1 \mathrm{~T}$ permanent

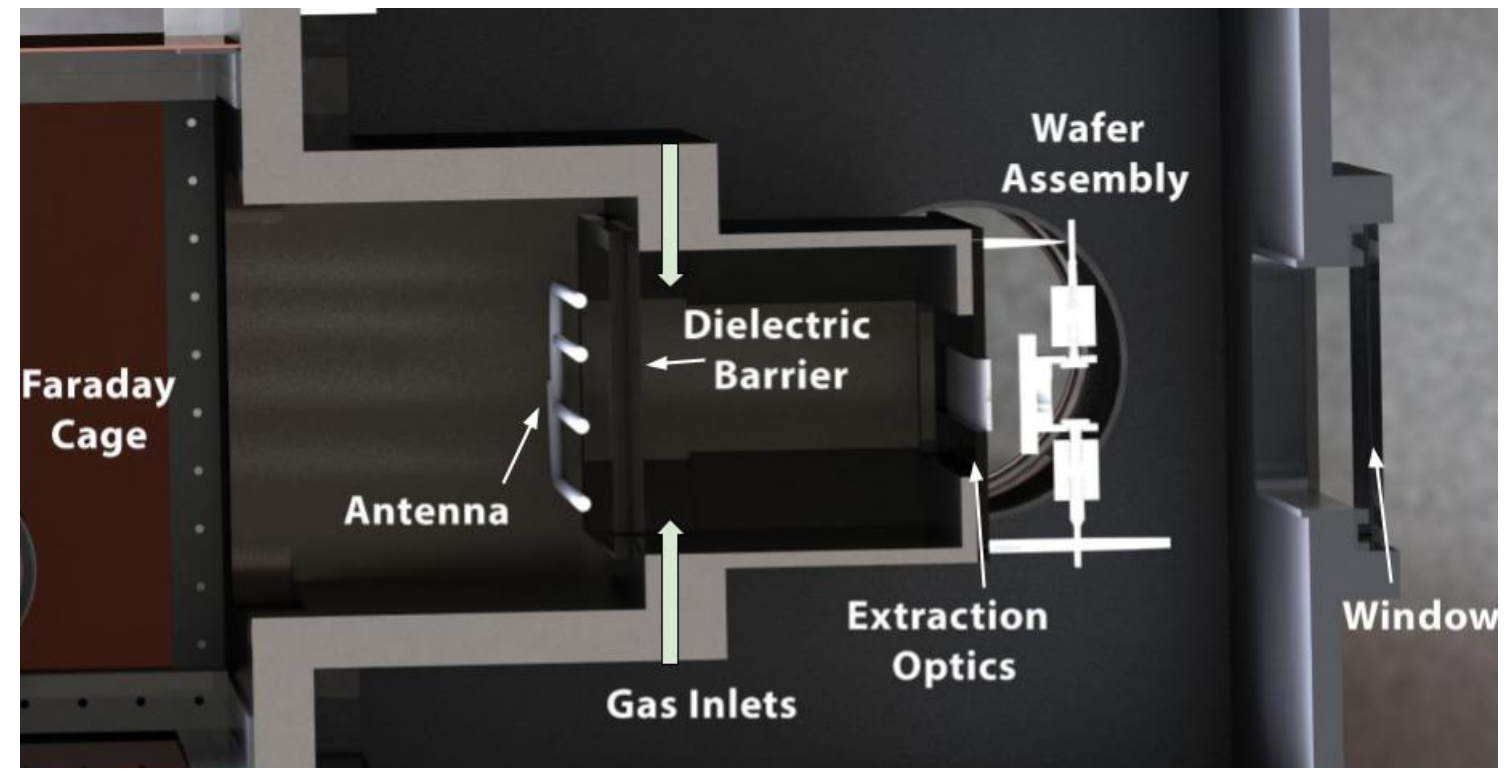

Figure 2.8: A bisected view of REVAN showing different interior parts. 
magnets arranged around the ionization region. This magnetic field ensures that only energetic particles are lost to the wall, improving ion density and plasma uniformity [75]. The ignition chamber is made of stainless steel and has an octagonal shape, measuring $24.00 " \times 5.56 " \times 4.18$ ". Opposite the dielectric barrier is an opening to the vacuum chamber that is covered by either quartz or graphite extraction optics. For the experiments described in this work, two different graphite optics were used.

\subsubsection{Slit Optics}

The extraction optics obtained from Applied Materials were modified to create specific extraction geometries. The slit aperture extraction optics is shown in Fig. 2.9. The opening of the graphite extraction optics is a $560 \mathrm{~mm} \times 30 \mathrm{~mm}$ rectangle. A graphite blocker, which totally covered the extraction slit, was placed behind the opening $5 \mathrm{~mm}$ into the ion source. This extraction optic was constructed such that the plasma from the source would diffuse out at a set angle in $-\hat{y}$ and replicates the wide area planar beam used in semiconductor processing.
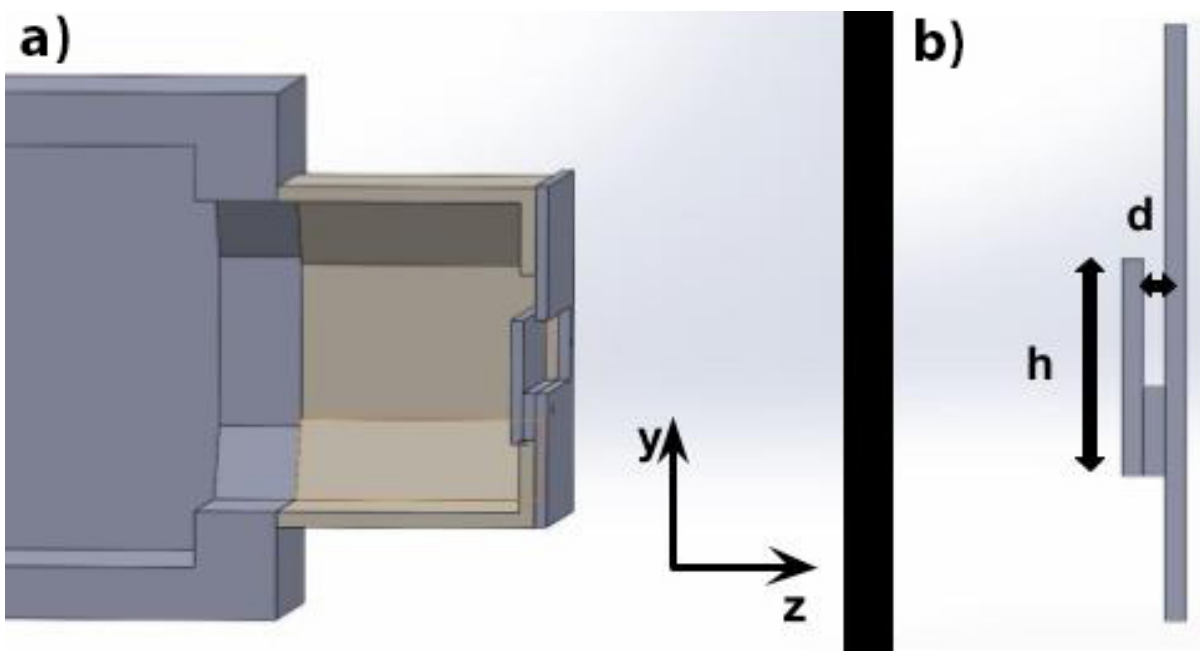

Figure 2.9: Extraction optics used in preliminary REVAN measurements. a) The slit extraction optics situated on the ion source. b) The profile view of the optics. The insertion of a graphite blocker between the extraction slit and the plasma forces the ions to exit at an angle. The blocker is $5 \mathrm{~mm}$ (d) from the extraction slit and has a height of $40 \mathrm{~mm}(\mathbf{h})$, fully blocking the extraction slit. 


\subsubsection{Aperture Optics}

For measurements of the plasma meniscus, different extraction optics were fabricated. These optics totally sealed the plasma in the ion source except for a $5 \mathrm{~mm}$ square hole that was milled into the center of the optics. This aperture allows for an extracted ion beam while being small enough to create a plasma meniscus. This extraction aperture was used for the majority of the work presented here. This configuration is shown in Fig. 2.10.

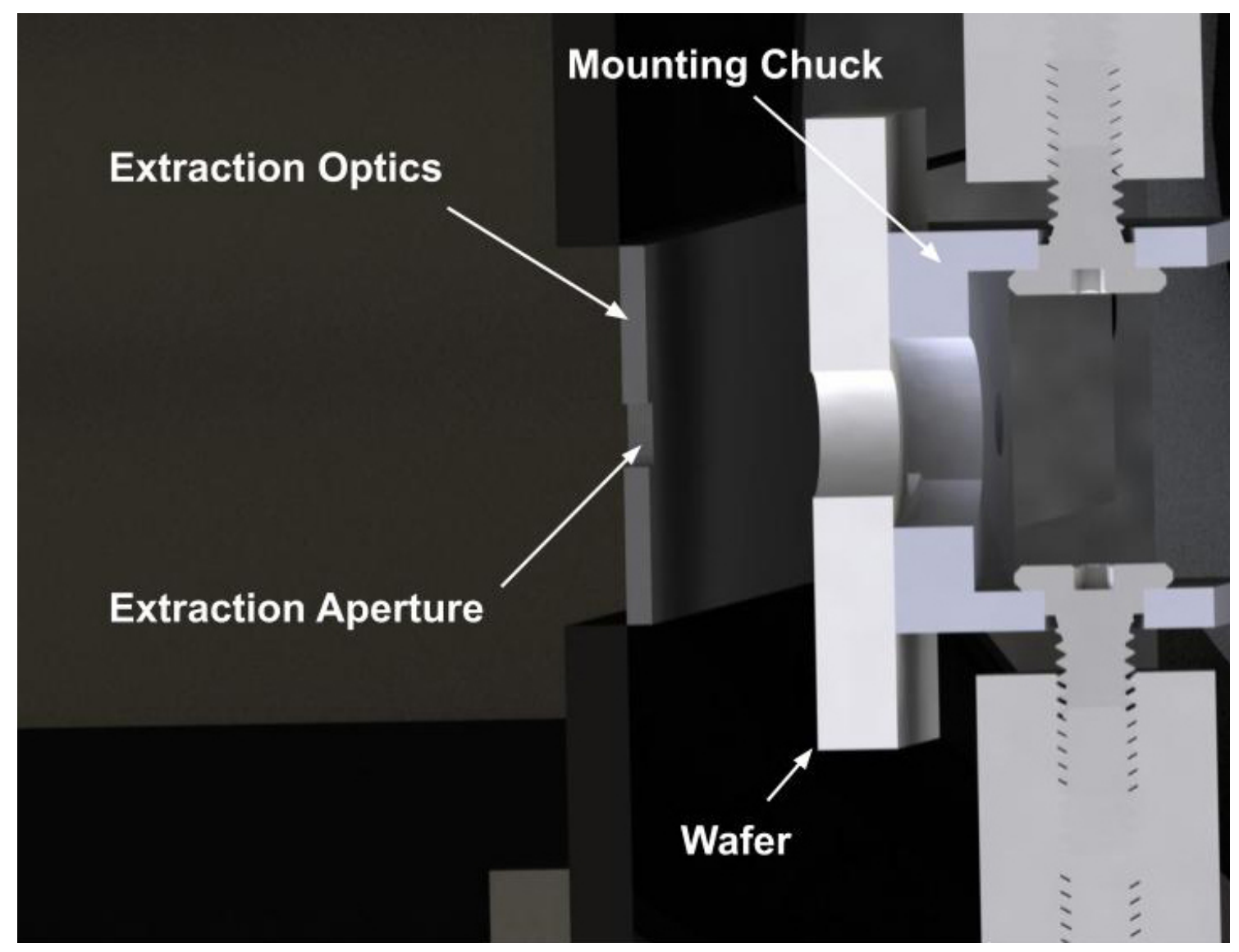

Figure 2.10: Zoomed in view of the interrogated region in REVAN. The small extraction aperture will create a continuous Debye sheath. The optical hole in the wafer was necessary to allow for the measurements to reach inside the source. 


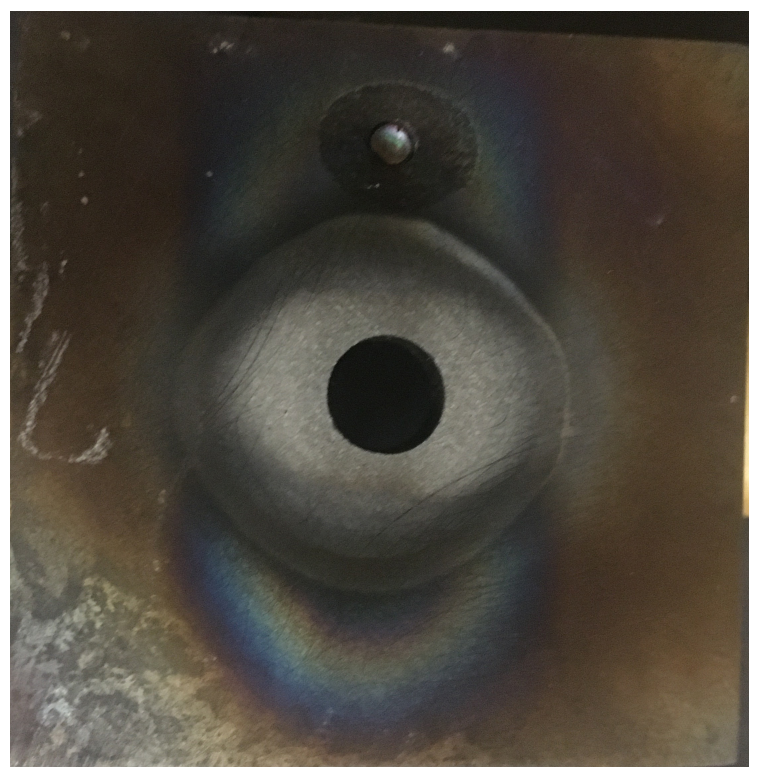

Figure 2.11: Image of the wafer used in experiments. Near the through hole, the wafer is discolored due to deposited metal from the source.

\subsubsection{Wafer}

For a wafer, REVAN uses a graphite square, $250 \mathrm{~mm}$ a side and $0.635 \mathrm{~mm}$ thick that is electrically isolated from the chamber. By applying a bias to this graphite, it acts similar to the wafers used in semiconductor processing, i.e., for ion extraction. The graphite is mounted to an aluminum chuck and the front of the wafer is positioned $12 \mathrm{~mm}$ from the face of the extraction optics, shown in Figure 2.11. The wafer assembly is shown in Fig. 2.8 in all white. An EG\&G 456 ORTEC High Voltage Power Supply sources the bias to the wafer by safe high voltage (SHV) feedthroughs located on a $\varnothing 2.75 "$ port.

This power supply is capable of producing up to $\pm 3000 \mathrm{~V}$ on the wafer. For the experiments detailed here, the applied voltage increased by increments of $1000 \mathrm{~V}$. The voltage was independently confirmed with a multimeter and was found to have an uncertainty of $\approx 4 \mathrm{~V}$. This error affects the potential by $<1 \%$ and is therefore ignored.

In order to provide optical access to the source chamber, an $\varnothing 8 \mathrm{~mm}$ hole was drilled in the graphite wafer. This provides a direct line-of-sight into the ion source 


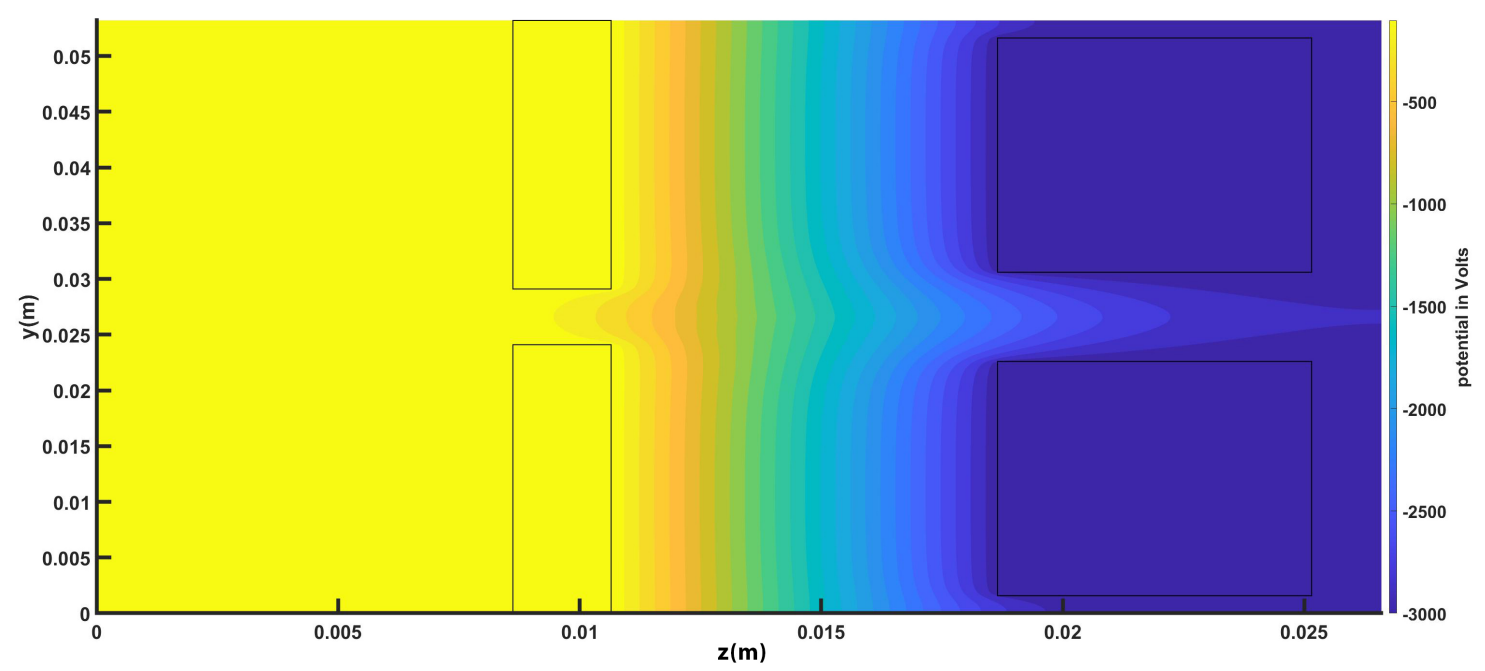

Figure 2.12: A map of the potential due to the biased wafer, generated by VSim 10.0. The potential is perturbed from a planar structure due to the hole drilled through the wafer. This perturbation damps out before the potential reaches the aperture.

from the front window of REVAN. The effect of this alteration on the potential structure is shown in Fig. 2.12. This potential map was created in VSim using a 2-d Poisson solver. A hole of this size creates a perturbation in the potential that is damped out within $5 \mathrm{~mm}$ of the opening. While this through hole is not ideal, it reduces the potential at the aperture by only $3 \%$.

\subsubsection{Pressure Management}

An $\varnothing 8 "$ side port connects to the vacuum system. This is comprised of a Balzers TMU 520 Turbo Pump and a Leybold backing diaphragm pump. These pumps are protected behind a gate valve. An interlock is implemented such that if the pressure reaches a critical level the interlock will activate and close the gate valve, isolating the pumps while the chamber is pressurized. These pumps maintain a base pressure of $\sim 5 \times 10^{-7}$ Torr.

These experiments were conducted at $\sim 1$ mTorr of argon gas. A constant flow rate of 0.3 SCCM was supplied to the chamber through an MKS 0-20 SCCM Mass Flow Controller. The gas is fed into the ion source by a gas manifold near the 


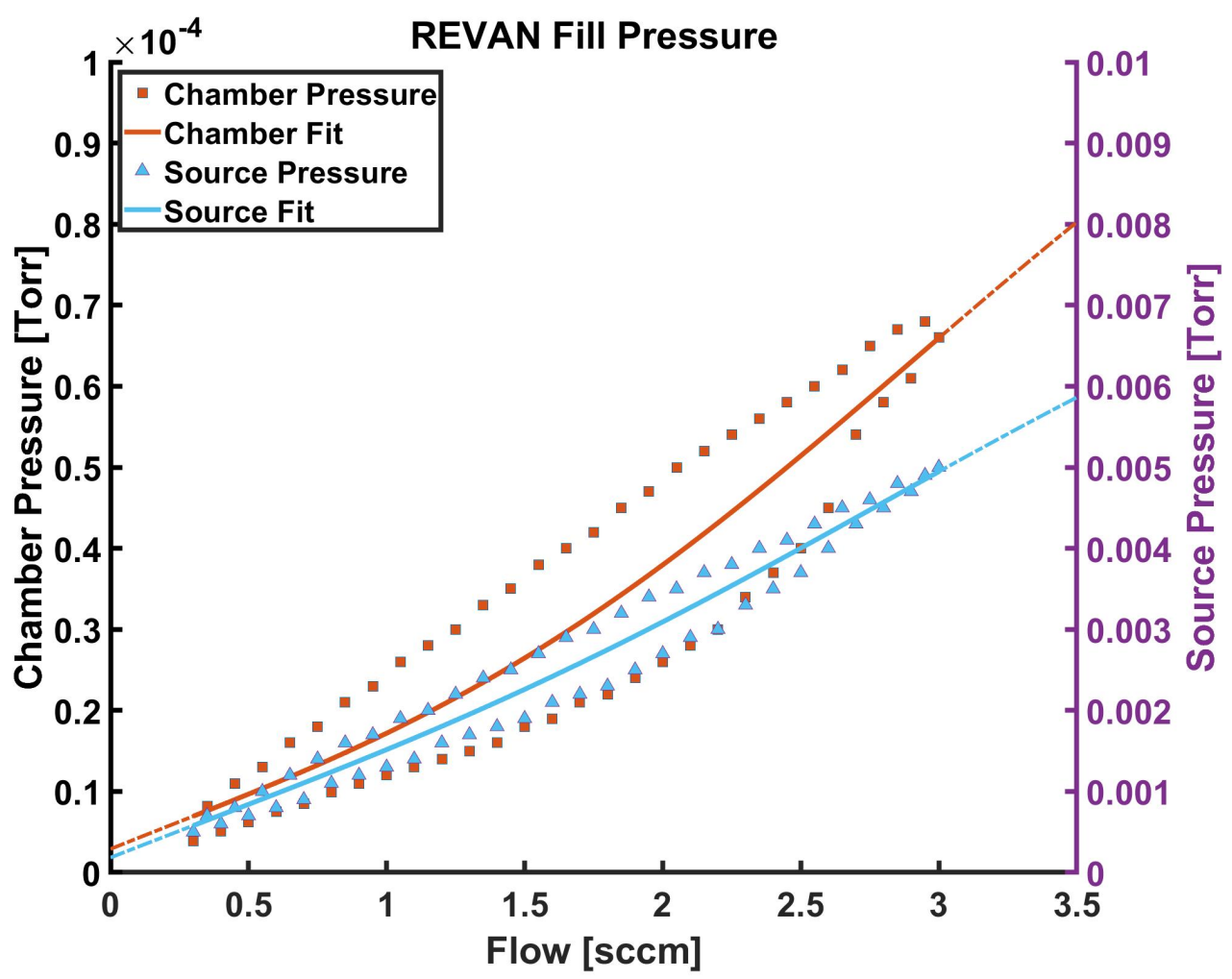

Figure 2.13: Comparison of simultaneous pressure measurements in the source and in the vacuum chamber. Data is fit with a least-squares algorithm to give a conversion from the vacuum pressure to an approximate source pressure.

dielectric barrier. The pressure is measured by a Pfeiffer Compact Full Range Gauge composed of a cold cathode and Piriani pressure gauge attached to a $\varnothing 2.75 "$ port on REVAN, far from the ion source. Therefore, the pressure given by this diagnostic is not representative of the pressure inside of the ion source.

When using the aperture optics, the ion source is sealed off from the pressure gauge except for the aperture. This creates a pressure differential between the source and chamber because of the conductance limitation of the aperture. Additionally, there is no port access to the ion source itself, preventing direct measurements of the pressure in the source during operation. To estimate a conversion between the source pressure and chamber, pressure a second Kurt J. Lesker KJLC CCPG Pirani pressure gauge was attached to an unused gas inlet. Pressure measurements were taken for neutral gas and the difference in pressure between the ion source and the vacuum chamber at the same mass flow is shown in Fig 2.13. Due to REVAN's high 
temperatures inside the Faraday cage, the pressure gauge would not survive during operation.

The pressure is recorded from $0.2 \mathrm{SCCM}$ to $3.05 \mathrm{SCCM}$ at increments of 0.1 SCCM. The pressure is also recorded from 3 SCCM back to 0.25 SCCM, filling in the points. Figure 2.13 shows a different pressure for nearby flow rate measurements despite the system relaxing for $\sim 2$ minutes between changes in flow. During REVAN operation, the pressure in the chamber did not change over many hours of measurements, except at high powers due to heating of the source. Lines of best fit to the pressure are plotted to allow conversion from the vacuum chamber pressure to the source pressure. This gives an uncertainty in the chamber pressure reading of $\delta P= \pm 0.00014$ Torr.

\subsubsection{Coordinate System}

Measurements were taken both inside and outside the plasma source. The maximum distance of measurements into the source was limited by the collection cone of the confocal optics (Section 3.2.2). $\hat{z}$ is taken to be in the direction of the propagating ion beam, with $z=0$ corresponding to the front face of the extraction optics. Therefore $z<0$ is inside the source and $z \geq 0$ is outside the source, in the vacuum chamber. $\hat{x}$ and $\hat{y}$ are transverse to the beam and their zeros are dependent on the extraction optics used. The confocal optical system only allows for measurements in the center of the beam $(\hat{x}=\hat{y}=0)$, but is capable of probing ions from $z=-25$ $\mathrm{mm}$ to $z=+12 \mathrm{~mm}$.

The measurements are organized into three regions:

- Deep inside the source, spanning from $-25<z<-10 \mathrm{~mm}$ where measurements are taken every $5 \mathrm{~mm}$. This is to measure the background plasma in which the ions are produced. 
- The aperture region, covering $-10<z<+1 \mathrm{~mm}$ in $1 \mathrm{~mm}$ increments. It is in this region that the meniscus is expected to form. Additionally, ions in this region are predicted to start feeling the effects of the Debye sheath.

- The extraction region, occupying the distance $1<z<12 \mathrm{~mm}$ is where the ion beam will propagate. Out of the source, the ions follow paths dictated by the potential structure created in the aperture region. 


\section{Chapter 3}

\section{Diagnostics}

\subsection{Laser Induced Fluorescence}

Particles that constitute the ion beam traverse three distinct regions as they are extracted from the source. Therefore, to measure these particles in a self-consistent way, the diagnostic used must also be able to spatially resolve ion dynamics in each region. Retarding field energy analyzers (RFEAs) are not capable of measuring ions in the source while Mach probes are very perturbative, destroying the potential structure of the meniscus, as well as over estimating the Mach number [76].

When studying plasma in regions with delicate potential structures, it is desirable to use non-perturbative techniques since these techniques preserve the integrity of the plasma. Laser induced fluorescence (LIF) is well suited to this end, as it selectively targets ions or neutrals without significant disruption of the plasma. This allows for valuable in situ measurements of the ion velocity distribution function (IVDF). Additionally, LIF is a robust diagnostic method that has become a well established technique for plasma spectroscopy after its introduction by Stern and Johnson in 1975 [77]. Since then, the technique has expanded to include two-photon laser induced fluorescence (TALIF) [78,79], planar LIF [80,81], and tomography [82]. 
This diagnostic is not without its limitations, as LIF can only target species with strong, identifiable transition states, ideally a ground state or metastable. Moreover, these transitions have to be accessible by laser wavelengths and the emitted photon should be at a wavelength with good detector efficiency. For these reasons, LIF schemes are commonly in the visible spectrum or near ultraviolet/infrared. Additionally, even if these conditions are met, the laser must have sufficient power and the plasma sufficient state density of the probed species to obtain reliable signal. Furthermore, LIF needs an unobstructed line-of-sight to measure a plasma volume. This can reduce its applicability for ion sources with little to no optical access. However, this limitation is relaxed by the use of confocal optics, which allow for injection and collection along the same path of optical access.

\subsubsection{Velocity Distribution Function}

LIF is performed by interrogating a section of the plasma volume with a narrow bandwidth laser. The laser wavelength is swept through a resonant transition of the target species. For the three-level LIF scheme used in this work, photons at the resonance wavelength are absorbed and excite an electron to a more energetic state. This excited state then decays to a third state, emitting a photon of known wavelength.

The intensity of the emitted photon is given by [83,84],

$$
\left.I_{L I F}(\nu)=\alpha+I_{\circ} \exp \left[-\gamma\left(\nu-\nu_{\circ}-\frac{u}{c} \nu_{\circ}-\delta \nu_{Z}\right)^{2}\right]\right]
$$

where $I_{L I F}$ is the intensity of the LIF signal at injected laser frequency $\nu, \gamma$ is a scaling factor $\gamma=m_{n} c^{2} / 2 k_{B} T_{n} \nu_{\circ}^{2}$ that relates particle temperature $T$ to mass, $\nu_{\circ}$ is the rest transition frequency for the species, and $\delta \nu_{Z}$ is the Zeeman shift. There are several broadening mechanisms which affect the measured temperature, discussed in Section 3.1.3, but typically Zeeman splitting and Doppler broadening dominate. 
$\alpha$ is an additional term to incorporate any DC offset from the collection electronics. Most important to the work reported here is the term $(u / c) \nu_{\circ}$, which is the Doppler shift due to the mean flow of the plasma $u$.

Due to the motion of the particle, the laser's wavelength in the frame of the absorbing particle will be different than the lab frame wavelength. If the particles are moving towards the injected laser, the wavelength that the particle "experiences" is blue-shifted, and if the particle is in motion away from the injection laser the wavelength is red-shifted from the lab frame. This is the well known Doppler shift. By sweeping the laser through a range of wavelengths, particles at rest and in motion are able to interact with the laser radiation. Because of this difference in lab and particle frame wavelengths, the LIF signal will vary according to the distribution of velocities in the plasma and give a velocity distribution function (VDF). Normalizing Eqn. 3.1 and converting to velocity space using the definition of the Doppler shift, $v=\left(\nu-\nu_{0} / \nu_{0}\right) c$, gives

$$
I_{L I F}(v)=\alpha+\exp \left[\frac{-\left(m / 2 k_{B}\right)(v-u)^{2}}{T}\right]
$$

where $\delta \nu_{Z}$ is ignored since REVAN's magnetic fields are too weak to cause Zeeman splitting. Obtaining a signal in the form of a Maxwellian distribution is desirable because then the properties of the species can be obtained by fitting a Gaussian function to the data. In the absence of significant line broadening mechanisms other than Doppler broadening, the full-width at half-maximum (FWHM) of the peak is proportional to the temperature $T$, the frequency offset between the peak and rest frequency measures the bulk flow $u$, and the area under the curve will give the relative state density of the transition. These ion properties are shown in Fig. 3.1.

Eqn. 3.2, however, only works with the assumption that there is a single population in the plasma, that it is in local thermodynamic equilibrium, and that the Doppler broadening dominates. This will produce a distribution with a Gaussian lineshape. If this is not the case, specie properties are given by the moments of 


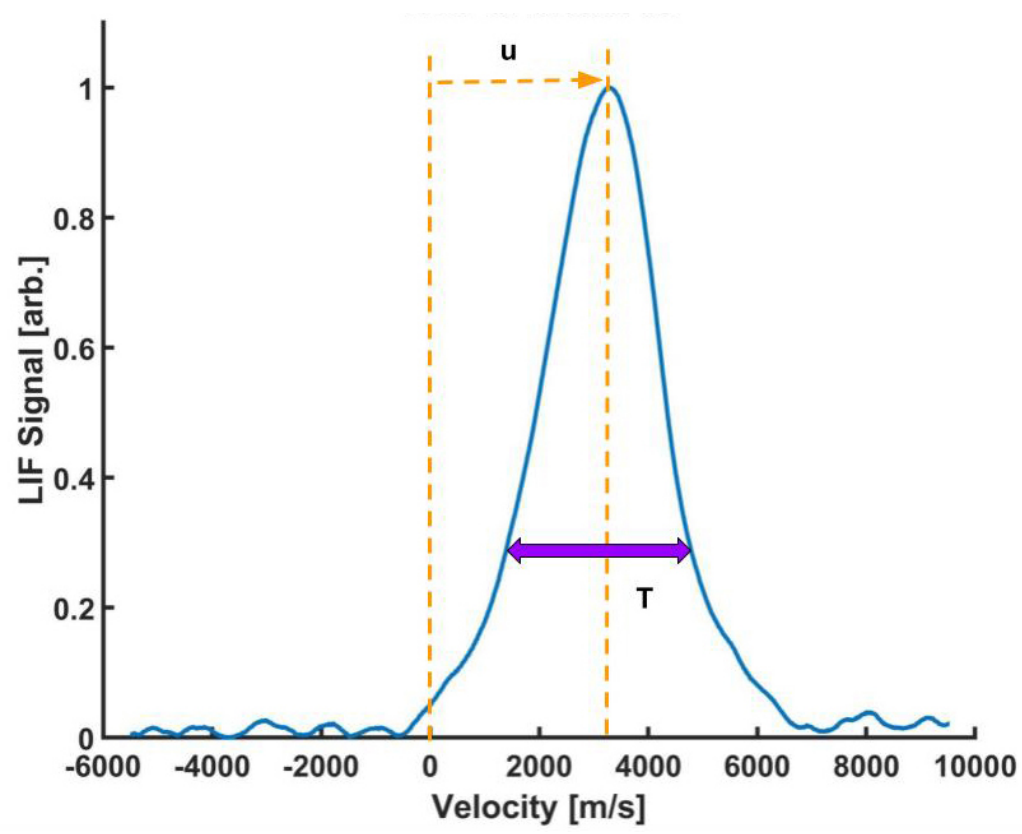

Figure 3.1: LIF data of an ion velocity distribution function showing how ion values are extracted from LIF signal.

the distribution. The zeroth, first, and second moments of the distribution give the relative density, bulk flow, and temperature, respectively:

$$
\begin{aligned}
\langle n\rangle & =\int_{-\infty}^{\infty} f(v) d v \\
\langle u\rangle & =\frac{\int_{-\infty}^{\infty} v f(v) d v}{\int_{-\infty}^{\infty} f(v) d v} \\
\frac{1}{2} m\left\langle u^{2}\right\rangle & =\frac{\int_{-\infty}^{\infty}(u-\langle u\rangle)^{2} f(u-\langle u\rangle) d(u-\langle u\rangle)}{\int_{-\infty}^{\infty} f(u) d u} .
\end{aligned}
$$

These moments give the average value of these quantities for the whole distribution and cannot distinguish individual populations in the distribution. For both Eqn. 3.2 and Eqn. 3.3, the density obtained is relative, proportional to the state density in the system. The LIF-measured density can be converted to an absolute density through the inclusion of a collisional-radiative model to determine the relative population of states and an end-to-end calibration of the system's light collection optics and detectors $[85,86]$. However, this method is difficult and involves additional diagnostics, such as Rayleigh scattering [87]. Densities are typically obtained through 


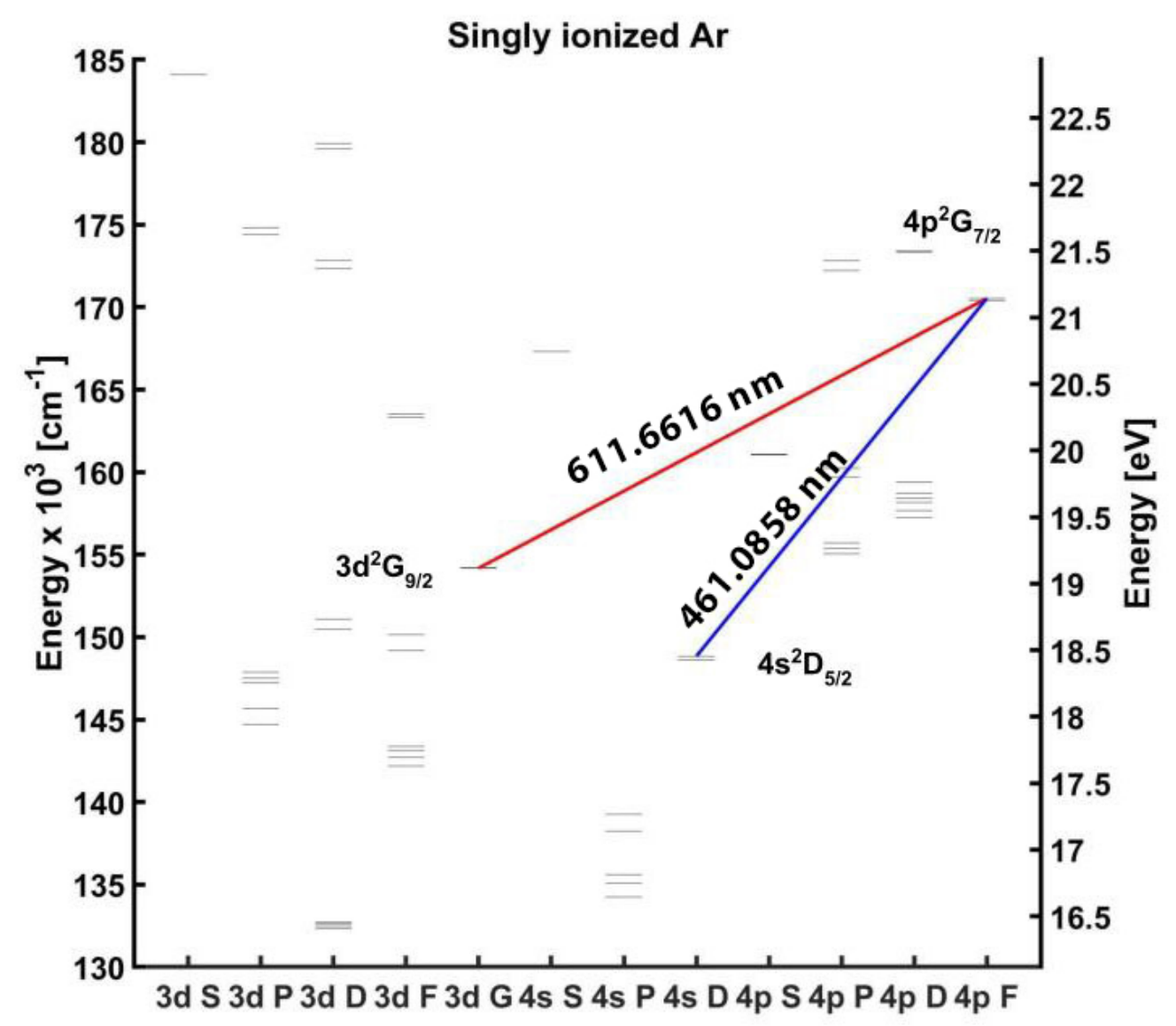

Figure 3.2: Partial Grotrian diagram of the LIF scheme for Ar II. The initial state $\left(3 d^{2} G_{9} / 2\right)$ is a metastable state that is excited by $611.6616 \mathrm{~nm}$ light. As the electron decays to the ground state it emits a $461.0858 \mathrm{~nm}$ photon.

other methods such as a Langmuir probe (with suitable assumptions of quasineutrality) or microwave interferometry [88]. TALIF is a promising, non-perturbative absolute density measurement alternative, provided the ground state is targeted and suitable calibration methods are available [89-91].

\subsubsection{Argon LIF}

Using LIF, IVDFs in an argon plasma are obtained by probing an Ar II metastable state. The scheme used is modeled after Goeckner et al. [92] and has been employed in many different areas of study at WVU [93-95]. To access this scheme, a collisionally excited, argon metastable ion in the $3 \mathrm{~d}^{2} \mathrm{G}_{9 / 2}$ state is pumped with 
$\lambda_{v a c}=611.6616 \mathrm{~nm}$ photons. The absorbed energy excites an electron to the $4 \mathrm{p}^{2} \mathrm{G}_{7 / 2}$ state, which then decays to the $4 \mathrm{~s}^{2} \mathrm{D}_{5 / 2}$ state and emits a photon with wavelength $\lambda_{\text {vac }}=461.0858 \mathrm{~nm}$. Figure 3.2 is a partial Grotrian diagram illustrating this process.

\subsubsection{Broadening Mechanisms}

While LIF has many advantages for probing ion and neutral species, care must be taken when interpreting the data. IVDFs are deceptively simple at first glance, but the analysis requires consideration of many different effects.

\subsubsection{Natural Linewidth}

The widths of spectral lines are not delta functions in frequency. Due to the uncertainty in energy $E$ and time $t$ the absorbed and emitted spectra will always have a natural width $(\Delta E \Delta t \geq \hbar / 2)$. This natural width is an atomic limit on how well a lineshape can be resolved. In the worst case, the absorbed linewidth can be large enough that the resulting IVDF peak becomes artificially broadened. The natural linewidth is proportional to the sum of all spontaneous emission coefficients from both the upper and lower states.

For the scheme used in this work, the spontaneous transition coefficients for

the Ar II $3 \mathrm{~d}^{2} \mathrm{G}_{9 / 2}$ metastable state are very small when compared to the excited state [96]. Therefore the natural linewidth is due only to the excited $4 \mathrm{p}^{2} \mathrm{G}_{7 / 2}$ state. This produces a natural line-halfwidth of the order $10^{-5} \mathrm{~nm}$. The uncertainty due to linewidth is therefore $\ll 1 \%$ and is ignored in analysis. 


\subsubsection{Pressure Broadening}

Pressure broadening is an umbrella term that covers many different mechanisms such as Van der Waals and resonance broadening. In a highly ionized plasma, the major contributor to this type of broadening is Stark broadening. This arises from an externally applied electric field or from localized electric micro-fields, which are pressure dependent. In both cases, a strong electric field affects the transition energy between split atomic levels. For micro-fields, their orientation is randomly distributed, causing broadening instead of resolvable transitions.

Stark broadening can both shift and broaden a peak, giving erroneous temperature and flow measurements. Griem gives expressions for the Stark effects at sufficiently low electron densities $\left(n_{e} \ll 1 \times 10^{20} \mathrm{~cm}^{-3}\right.$ at $\left.T_{e} \approx 5 \mathrm{eV}\right)$ [97]. These equations results in a peak shift of order $10^{-4} \mathrm{~nm}$ and a broadening of order $10^{-5}$ $\mathrm{nm}$. Both of these introduce an error of less than $1 \%$ and are ignored for the measurements presented here.

The externally applied electric field in REVAN is not strong enough to produce significant Stark broadening. Experiments investigating the external field broadening of Ar II have been carried out up to $20 \mathrm{kV} / \mathrm{m}$, with modest shifts and broadening [98]. Gavrilenko et al. were unable to resolve any Stark effect on argon ions that did not transition to a high principle number $(n \geq 8)$ [99]. Therefore, Stark shifts are not considered in this work.

\subsubsection{Zeeman Broadening}

Zeeman broadening arises from the coupling of an ion's magnetic moment to an external magnetic field. This results in the splitting of observed spectral lines, very similar to the Stark broadening. While REVAN has a strong magnetic field capable of creating a Zeeman effect at the wall of the source, the field drops to negligible levels at the measurement location. Therefore, Zeeman broadening is not appreciable for 
these measurements.

\subsubsection{Laser Power Broadening}

An absorption line is broadened when more electrons are being excited than are decaying to the ground or metastable state. This leads to an underdeveloped peak and enhanced edges of the distribution. Near the peak of the distribution the metastable state becomes depleted, and signal is no longer proportional to the injected laser power. Also known as saturation broadening, this mechanism is a well-documented issue in single photon LIF [100-102].

This mechanism is a potentially significant source of broadening in REVAN. Due to the low metastable density in REVAN, an appropriate laser power needs to be injected in order to avoid an artificially broadened lineshape. There are several models to estimate the amount of broadening based on laser intensity [96,103]. Practically, however, it is much more useful to experimentally determine the onset of power broadening. If power broadening is an issue in the system, the ion temperatures appear artificially larger. By reducing the injected laser power and taking measurements at the same location and conditions, the FWHM of the peak will decrease if the lineshape is broadened. Eventually, the laser power is set low enough that the metastable state is no longer saturated. Figure 3.3 shows the effects of this type of broadening mechanism on IVDFs and temperature. In order to prevent this type of broadening in these measurements, the power injected into the fiber was kept at $\sim 50 \mathrm{~mW}$ (Section 3.2.1).

\subsubsection{Doppler Broadening}

Doppler broadening arises when the absorbing species have random thermal motion. The particle motion will have components that are parallel and anti-parallel to the injected laser path. This will shift the transition wavelength in the laboratory frame 

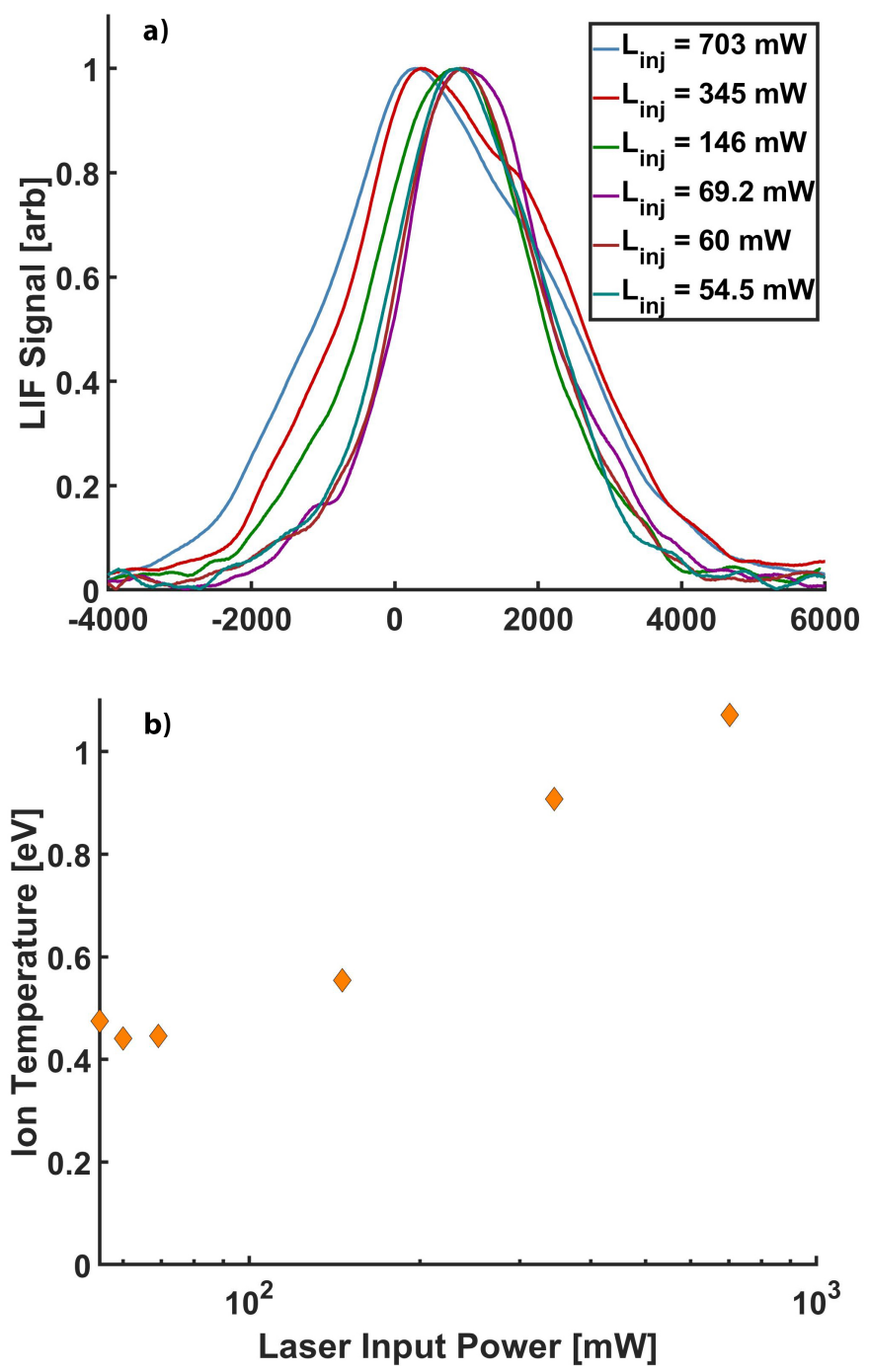

Figure 3.3: Effects of power broadening on IVDF measurements. a) The peak becomes narrower with lower injected laser power. b) A semi-log plot of the ion temperature verses injected laser power. From this an appropriate injected laser power is found that maximizes signal without broadening. In this case, the three measurements below $10^{2} \mathrm{~mW}$ are not saturated.

based on the velocity of the particle. The Doppler shift is given by

$$
\Delta \omega=\vec{k} \cdot \vec{v}
$$

with $\Delta \omega=2 \pi \Delta \nu$, where $\Delta \nu$ is the observed frequency shift from the resonant frequency, $\vec{k}=(2 \pi / \lambda) \hat{k}$ is the wave vector of the injected laser, and $\vec{v}$ is the velocity 
of the absorber [103]. With some simple algebraic manipulation, Eqn. 3.6 becomes

$$
\begin{aligned}
\Delta \nu & =\nu^{\prime}-\nu_{0} \\
& =\frac{\vec{k}}{2 \pi} \cdot \vec{v} \\
& =\left(\frac{1}{\lambda_{0}}\right) \hat{k} \cdot \vec{v} \\
& =\frac{\nu_{0}}{c} v \\
\Rightarrow \nu^{\prime} & =\nu_{0}\left(1+\frac{v}{c}\right),
\end{aligned}
$$

where $\nu^{\prime}$ is the shifted frequency. Equation 3.7 is the linear Doppler shift and only holds for $v / c \ll 1$. While the maximum speeds measured in REVAN are large, the ratio is still sufficiently small $\left(v_{\max } / c \approx 6.0 \times 10^{-4}\right)$ and the linear Doppler analysis is valid.

Doppler broadening is exploited in LIF analysis as it gives the random thermal motion, or temperature, of the probed species. Additionally, this broadening mechanism often dominates the IVDF width. The half width for Doppler broadening of argon gas is [96]

$$
\Delta \lambda_{d, 1 / 2}=7.46 \times 10^{-2}(T)^{1 / 2} .
$$

Here $\lambda$ is given in nanometers and $T$ is given in eV. For the typical ion temperatures measured inside REVAN, the Doppler broadening is on the order of $10^{-3} \mathrm{~nm}$, making it the dominant broadening mechanism. Doppler broadening, therefore, is at least an order of magnitude larger than the next largest broadening mechanism.

\subsubsection{Metastable Quenching}

The $3 \mathrm{~d}^{2} \mathrm{G}_{9 / 2}$ metastable state is populated through collisions. While this state lies $19.12 \mathrm{eV}$ above the ground state, not all of the energy needs to come from electron-ion collisions [104]. While electron-ion collisions dominate the ionization 
and metastable creation, a metastable state can also be achieved by Coulomb collisions between ions in different electronic states [105] as well as through step-wise excitation [106].

The LIF scheme described here probes a metastable state. Assuming electronion collisions are the dominate mechanism, LIF signal is dependent on the density and electron temperature of the plasma such that [107]

$$
I_{L I F} \propto \sqrt{n^{2} T_{e}}
$$

where

$$
n^{2}=n_{i} n_{e} \approx \frac{n_{j}}{\langle\sigma v\rangle_{0 j}} \sum_{i<j} A_{i j}
$$

Here $n$ is the plasma density, $n_{j}$ is the density of ions in the metastable state $j,\langle\sigma v\rangle_{0 j}$ is the velocity distribution averaged cross section for electron impact excitation from the ion ground state to $j$, and $\sum_{i<j} A_{i j}$ is the sum of the spontaneous transition rates from the metastable state to all lower states [97].

Once excited, the ions will remain in the metastable state for an average lifetime before decaying back to the ground state. The lifetime of the metastable state probed here is difficult to measure, but recent works have employed the use of wave-particle interactions to determine a lifetime of $t_{A r I I^{*}} \approx 18 \mu \mathrm{s}[105,108]$. In addition to simply decaying, particles in a metastable state can be forced into states without emission at $461 \mathrm{~nm}$ through interactions with the plasma. These quenching processes are [109]:

1. Diffusive losses to the wall.

2. Two-body collision with neutral atoms. These can result in;

- excitation to a higher state. 
- de-excitation to a lower state.

- formation of an unstable diatomic molecule and collision induced radiative transitions.

3. Three-body collision with two neutrals and production of a stable, excited molecule.

4. Collisions between a pair of metastable atoms resulting in an ionization of one and de-excitation of the other.

Therefore, to ensure sufficient LIF signal, ample metastable state density is essential and metastable quenching mechanisms must be mitigated.

In REVAN, LIF signal decreased with increasing source pressure, suggesting metastable quenching at higher pressures. Equation 3.9 shows that an increase in pressure, and therefore plasma density, should result in an increase in metastable density and signal, assuming constant ionization. Therefore, the observed decrease in signal suggests the metastable state is quenched before it is measured.

Equation 3.9 is also proportional to electron temperature. In ICPs, as pressure decreases electron temperature increases. This is due to the anomalous skin effect which is associated with preferential collisionless heating (Section 2.2.2) [110]. Therefore a lower source pressure produces more electrons with energies capable of exciting ions to the probed metastable state. Therefore an operating pressure of $\sim 1$ mTorr was chosen for these experiments.

The collisional quenching rate $r_{Q}$ for $\mathrm{Ar}$ II $3 \mathrm{~d}^{2} \mathrm{G}_{9 / 2}$ is given as [105]

$$
r_{Q}=n_{n} \sigma \sqrt{\frac{8 k_{B} T_{n}}{\pi m_{n}}}
$$

where $n_{n}$ is the neutral density, $\sigma$ is the quench cross-section, $T_{n}$ is the neutral temperature, and $m_{n}$ is the mass of the neutral particles. So as the pressure increases so will the collisions in the system. As collisions increase, the probability that 
a metastable state is depopulated increases. Additionally, an increase in pressure results in a decrease in the electron mean free path, limiting the energy electrons can gain from the field, as well as increasing the electron induced collisional quenching [111]. Therefore, quenching prevents high pressure LIF measurements in REVAN.

\section{$3.2 \quad$ Optics}

\subsubsection{Beampath}

A schematic of the laser beampath is given in Fig. 3.4. The injection beam is produced by a $10 \mathrm{~W}$ Nd:YAG pump laser and a Sirah Matisse DR (dye ring) laser. A Rhodamine 590-Ethylene Glycol dye solution converts the green $532 \mathrm{~nm}$ pump laser to $611 \mathrm{~nm}$ red light. The laser outputs a peak power of $\approx 1.5 \mathrm{~W}$ at $\lambda_{\text {vac }}=611.6616$ nm.

When the laser exits the cavity it is split by a 90:10 optical blank. Of the $\approx 10 \%$ laser power taken from the main beampath, $90 \%$ is sent to a room temperature

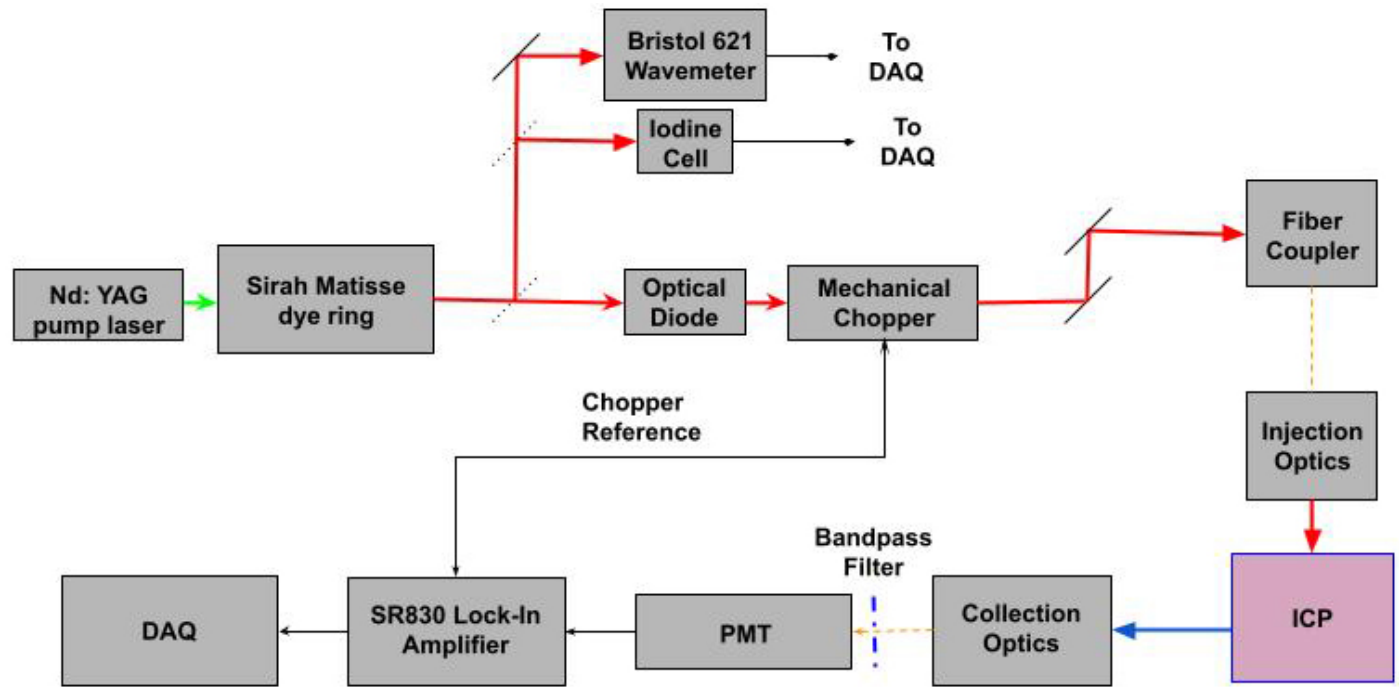

Figure 3.4: The injection and collection path for LIF. This setup is consistent between both optical methods used with the exception of the injection fiber and the use of a neutral density filter. 
iodine cell and the other $10 \%$ is fiber coupled to a Bristol 621 wavemeter. The wavemeter provides real-time measurements of the laser's wavelength while the laser is sweeping. The wavemeter has an accuracy of $\delta \lambda_{\text {las }}= \pm 0.0001 \mathrm{~nm}$ which results in a velocity error of $\delta v_{\mathrm{WM}} \approx 50 \mathrm{~m} / \mathrm{s}$. The iodine cell produces well defined absorption peaks used for frequency calibration.

The other $90 \%$ of the laser power travels along the main injection path. After the beam splitter, the laser beam passes through an Optics For Research IO-5633-PBS optical diode. This optical diode only transmits $83 \%$ of laser power, but safeguards the Matisse from damaging reflections. The laser beam then passes through a mechanical chopper which modulates the laser beam at a frequency of 5 $\mathrm{kHz}$. After modulation, the laser is reflected off two steering mirrors with $\geq 98 \%$ reflectance each before coupling into a fiber port.

After the fiber port, the laser can travel by two different types of optical fibers, depending on the choice of injection optics. Single mode (SM) fibers have a relatively low transmittance $(\leq 50 \%)$ due to their core size of $\varnothing 5-10 \mu \mathrm{m}$. While this limited transmitted laser power to $\approx 33 \%$ out of the fiber, the benefit is a much better Gaussian, point-like, beam from the fiber. This allows the injected laser to maintain better beam properties, specifically beam diameter, which results in better spatial localization. Multimode (MM) fibers, which have much larger cores of $\varnothing 200 \mu \mathrm{m}$ or more make alignment easier, and have a power transmission of $\approx 80 \%$, but with a trade-off of a rapidly diverging beam.

The light emitted from the plasma is coupled into a $\varnothing 1 \mathrm{~mm}$ core MM fiber by collection optics. It is advantageous to use a large core fiber for collection in order to maximize the collected signal even at the cost of spatial localization. The $461 \mathrm{~nm}$ fluorescence is transmitted to a Hamamatsu HC 120-05MOD IR-sensitive photomultiplier tube (PMT). Fluorescence signal is selected from the background emission by a $1 \mathrm{~nm}$ FWHM bandpass filter centered at $461 \mathrm{~nm}$ and has a maximum transmission of $\approx 72 \%$. The PMT converts the collected light into an electrical 
current and sends it a Stanford Research Systems SR830 lock-in amplifier. The lock-in amplifier is referenced to the mechanical chopper such that only signal at the modulated frequency $(5 \mathrm{kHz})$ is recorded. Uncorrelated background emission is rejected. For an in-depth explanation of lock-in amplifiers the reader is directed to Reference [112].

\subsubsection{Confocal System}

The primary optical diagnostic tool in this work is the confocal telescope. This optical arrangement benefits from concentric injection and collection paths, allowing for LIF measurements along a single axis. This feature is immensely beneficial because it requires only one point of optical access, allowing measurements in previously restricted regions of the plasma [114]. This is achieved by making the collection cone hollow and injecting down the center of it. This is beneficial in that line integration of signal is reduced since the injection and collection only overlap at the focal plane, increasing spatial localization. The trade off is that a hollow profile reduces signal, resulting in a lower signal-to-noise ratio (SNR).

Figure 3.5 shows the injection path (red) and the collection path (blue) for the confocal apparatus. Laser light is delivered to the system by a SM fiber. The

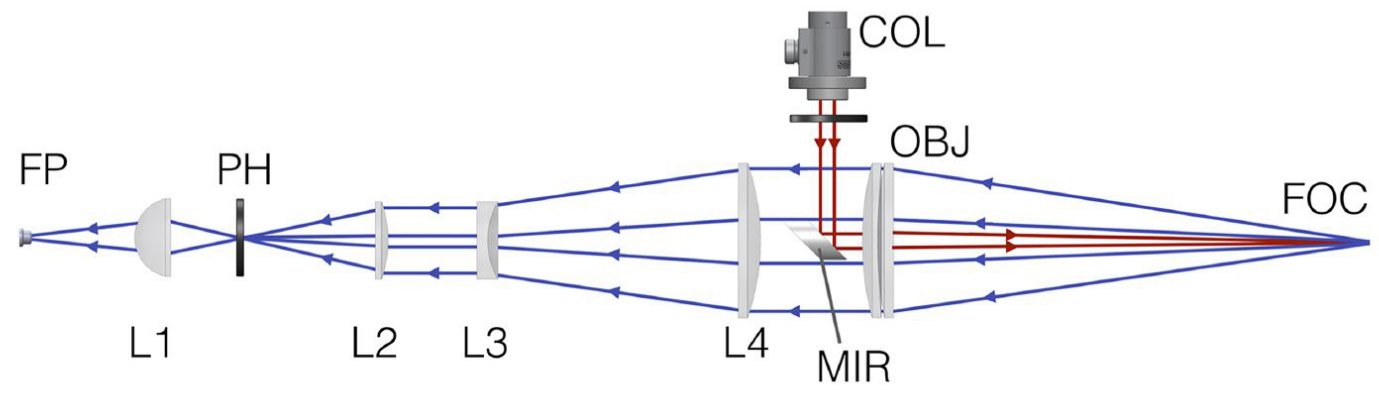

Figure 3.5: Ray tracing diagram for the confocal telescope system. The components are as follows: collimator (COL), mirror (MIR), focusing lens (OBJ), focal point (FOC), compression lens (L4), diverging lens (L3), pinhole lens (L2), pinhole (PH), fiber lens (L1), fiber port (FP). In this arrangement the mirror doubles as the obstruction. Reproduced with permission from Ref. [113], all rights reserved. 
light exits the fiber into a $2 \mathrm{~mm}$ reflective collimator, travels to a turning mirror that redirects the beam $90^{\circ}$ into a $\varnothing 2 "$ plano-convex focusing doublet lens. This arrangement keeps the injection beam diameter small with minimal divergence so that at the focal plane there is point-like illumination. However, using an SM fiber can lead to power broadening. At the focal point of the beam the power density is sufficient to saturate the ion metastable transition. To avoid this, a neutral density filter (NDF) was placed before the fiber port and reduced the coupled laser power by $\approx 85 \%$. With this in place, the typical power deliver to the plasma was $\approx 4 \mathrm{~mW}$.

The emitted fluorescence is isotropic. Therefore a subset of the emitted photons are collected by the focusing lens's solid angle. Collected light passes through the doublet and is collimated before being compressed and focused into a MM collection fiber. The amount of light incident on the collection fiber is determined by the size of the obstruction, which blocks the part of optical path to create the hollow collection profile, and the pinhole. Together these two components create a collection annulus.

The geometric properties of this annulus are significant in determining the spatial localization of the system. Shown in Fig 3.6 is the injection beam and back-lit collection path at and near the focal plane. The outer diameter of the annulus is determined by the pinhole while the inner diameter is determined by the size of the obstruction. Having a thicker annulus will collect more signal, but at the cost of spatial localization. An approximation of the depth of field and spatial localization for this system is given in the appendix of Reference [113]. This optical arrangement is attached to a linear stage with an external position reference, giving it a position uncertainty of $\delta z= \pm 0.28 \mathrm{~mm}$.

Practically, the spatial localization is found using a beam profiler. Far from the focal plane the annulus and injection beam are two, distinct structures. Closer to the plane they begin to overlap until they are focused at the same point. Despite beginning to overlap before the focal plane, little to no signal will be collected since the energy density in the wings of the Gaussian beam is low. An annular collection 


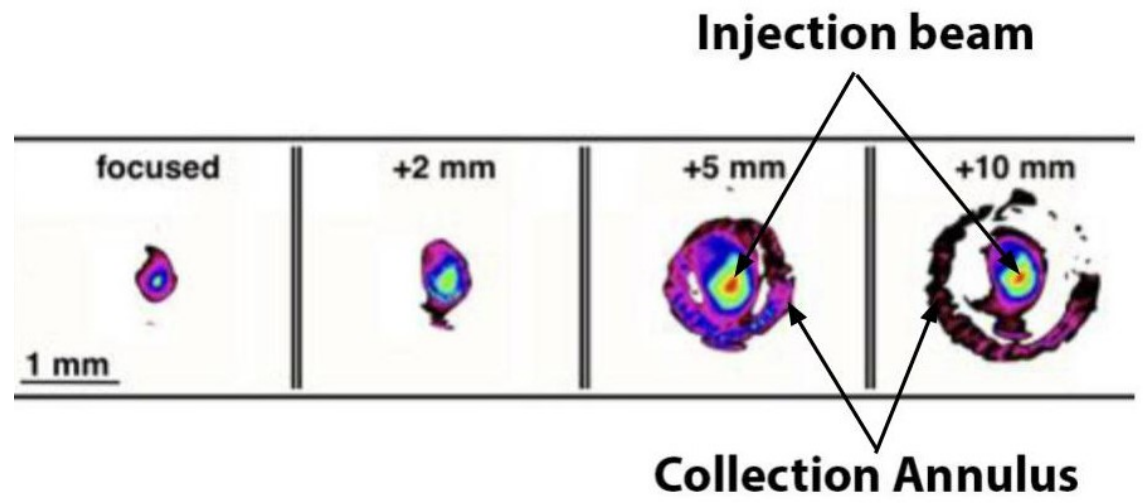

Figure 3.6: Beam profile and collection annulus profile after the doublet lens in the confocal telescope at various lengths. The collection annulus will start to overlap the injection beam closer to the focal plane so that there is minimal line integration of LIF signal. Reproduced with permission from Ref. [113], all rights reserved.

cone reduces line integration, making the focal point so spatially sensitive that the injection beam must precisely overlap the annulus for signal to be observed with the confocal system. This optical arrangement has sub-millimeter resolution at the focal plane [100]. However, in REVAN the system was signal starved, necessitating a $\varnothing 100 \mu \mathrm{m}$ pinhole with a obstruction diameter of $\approx 1.2 "$. This gave a spatial localization of $1 \mathrm{~mm}$ and a depth of field of $\approx 2 \mathrm{~mm}$.

It should be emphasized that the work done here is only possible with the use of this confocal system. Since LIF is performed along a single axis, the focal plane lies within the plasma source. Additionally, the non-perturbative nature of LIF allows for in situ measurements of the ion properties at and near the aperture. The maximum measurable depth in REVAN is $25 \mathrm{~mm}$ past the front of the aperture. This limit is set by the focal length of the system. For distances more than $25 \mathrm{~mm}$ into the source, the collection cone is obstructed by the aperture. Theoretically, the entire depth of the source is accessible by the confocal system by increasing the focal length. However this leads to decrease in spatial localization at the focal plane from optical aberrations and less signal is obtained due to a smaller fraction of the collection solid angle [83]. 


\subsubsection{Conventional Optics}

An orthogonal configuration of injection and collection paths is the standard for LIF measurements. In this way, the injection and collection optics are uncoupled, and it is much simpler to align. This crossing optical arrangement was used when measuring the plasma outside of the aperture. Figure 3.7 shows the $2 \mathrm{D}$ stage used in this experiment where injection and collection optics are connected to linear motion stages and, once initially aligned, allow for simple, repeatable translation. Optical alignment of this system is achieved without the need of a beam profiler. While this system provides much better signal, it decreases the spatial localization to $\approx 2 \mathrm{~mm}$ at the focal plane. Additionally, this system does not have a hollow collection profile resulting in line integration for the entire overlap of the collection and injection optics. Therefore, the optics pick up more contributions from ions near, but not at, the focal plane. This arrangement uses the same external reference as the confocal system and therefore has a position uncertainty of $\delta z= \pm 0.28 \mathrm{~mm}$.

The injected light is carried to the 2D stage by a $\varnothing 200 \mathrm{MM}$ fiber and is collimated at the stage by a ThorLabs RC02SMA-P01 reflective collimator. The beam diverges rapidly due to the non-Gaussian nature of the MM. Additional lenses were added to the beampath to try and preserve collimation. The larger injection diameter also circumvents the power broadening issue since the energy density is much less when using MM fibers. Therefore, there is no need for an NDF in the beampath. Collection optics incorporate a $\varnothing 2$ " doublet lens that focuses fluorescence into a MM collection fiber for transport to the PMT. By swapping the cage mounted lenses, injection in both $\hat{z}$ and $\hat{y}$ were performed. Due to the different distances to the measurement region each configuration had a unique combination of lenses to achieve the correct focal length.

A larger injection beam and collection optics increases the interrogated volume, leading to less spatial localization. Ion beam dynamics often occur over small scales 


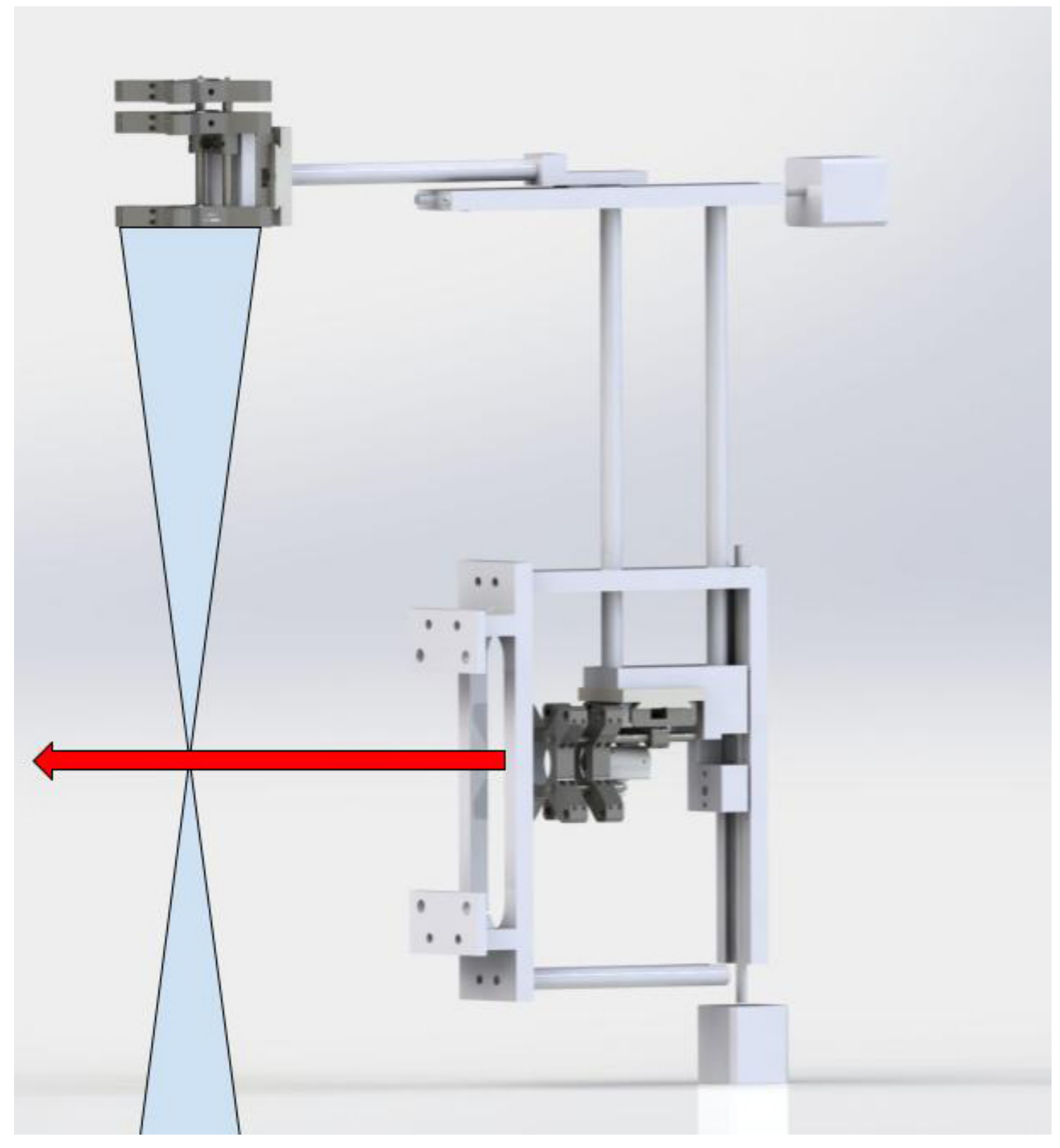

Figure 3.7: The optical arrangement used to obtain IVDFs outside of the ion source. The collection cone is shown in blue and the injected laser is shown in red.

[115] and poor localization can also lead to broadening due to signal averaging over a larger volume at the measurement location. If an ion beam is converging or diverging, then the ions will have a radius dependent, radial velocity $(\hat{y})$. Therefore, if the $2 \mathrm{D}$ stage is measuring a plasma volume exactly in the middle of the beam, but the collection cone is sampling above and below, ions traveling both towards and away from the injected laser will contribute to the LIF signal. This will produce a double Maxwellian distribution that is difficult to separate when the ion velocities are 


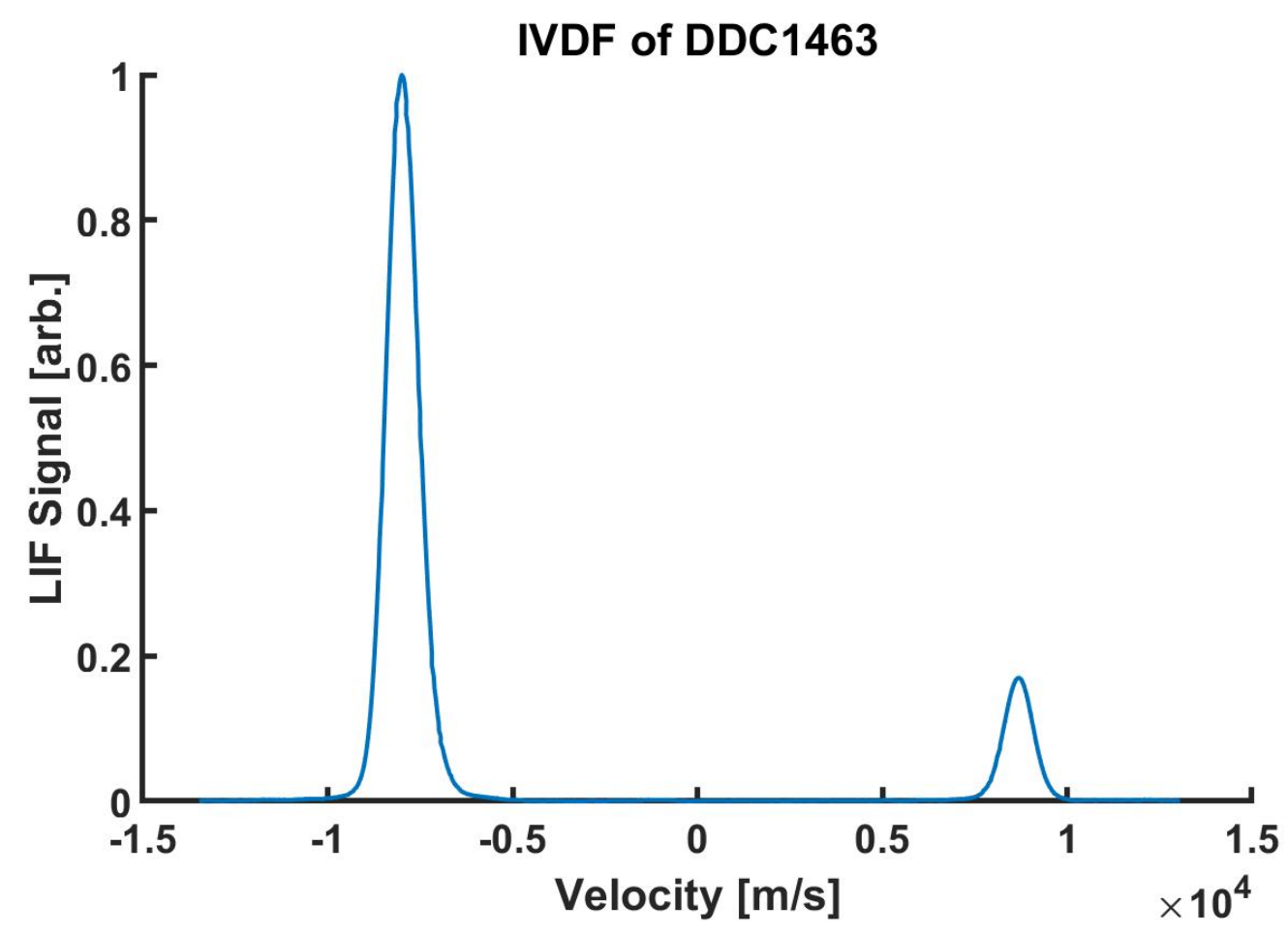

Figure 3.8: IVDF obtained by the 2D stage in $\hat{y}$. The two peaks indicate that the injection beam is reflecting off the far window and traveling back through the plasma. The reflected beam has the same focal plane as the injected beam, but has a fraction of the power and an opposite wavevector. This causes a second peak to appear reflected about zero and with much less signal.

low velocities and can appear as a single, broad population. Fortunately, in REVAN the velocities are large enough that the peaks are clearly separated. Reflections can also cause a double Maxwellian distribution, as shown in Fig. 3.8, but these cases are easy to distinguish due to the degree of separation between the peaks.

\subsection{Langmuir Probe}

Langmuir probes are one of the oldest plasma diagnostics and were first demonstrated by Langmuir and Mott-Smith in 1924 [116,117]. The physical design of this diagnostic is beguilingly simple. It is, at its core, a wire that is stuck into the plasma and biased. However, the analysis of the collected current is still a very active area of study [118]. An excellent overview of the different analysis techniques is given in Reference [83]. 


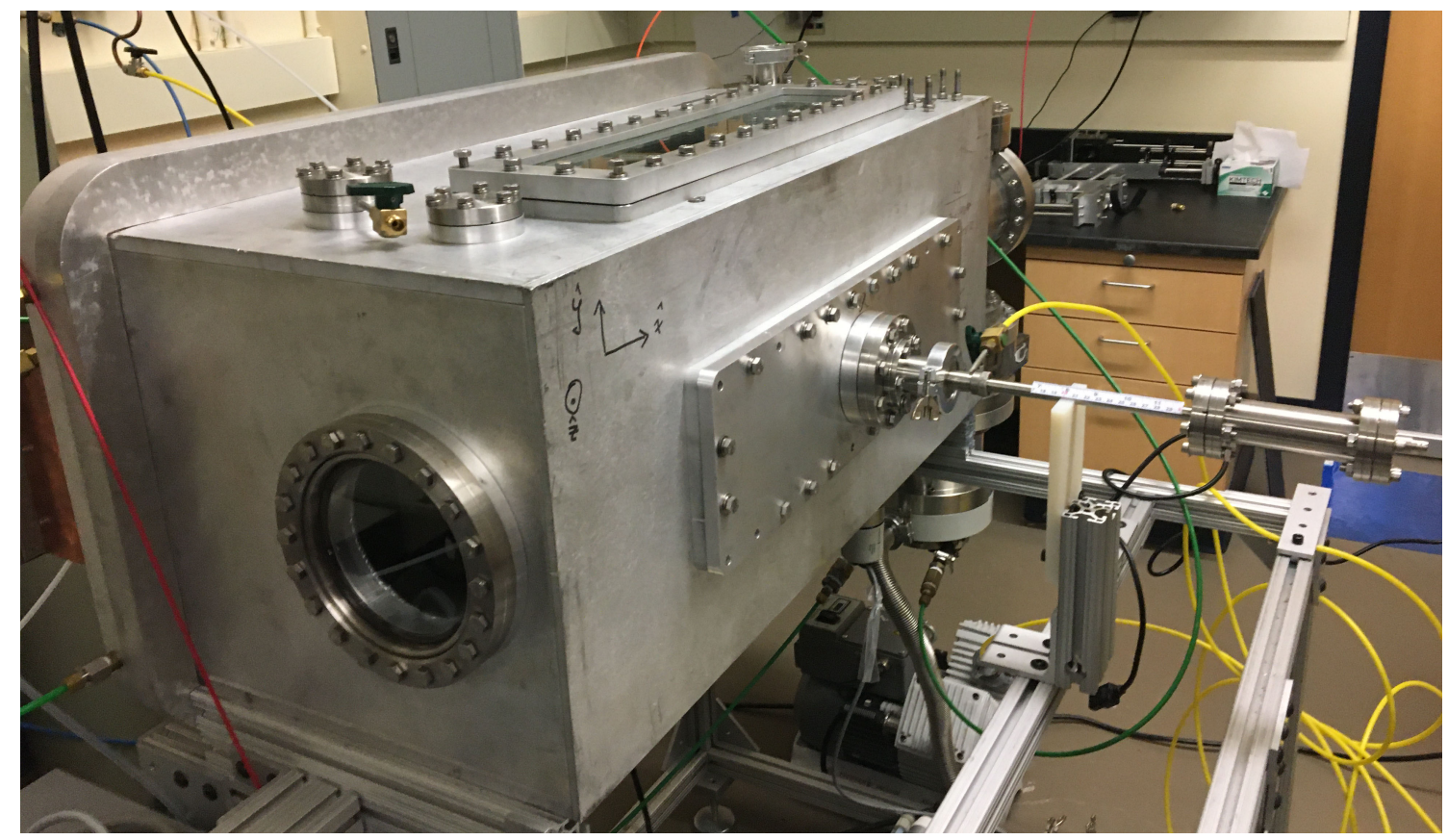

Figure 3.9: Image of the Langmuir probe assembly mounted on REVAN. It is centered on the extraction aperture, such that $x=y=0$ and translates in $\hat{z}$.

\subsubsection{Probe Construction}

The probe tip consists of a length of $\varnothing 0.5 \mathrm{~mm}$ graphite soldered to a brass connector. The graphite is shielded from the plasma by a casing of alumina $\left(\mathrm{Al}_{2} \mathrm{O}_{3}\right)$ leaving only $2 \mathrm{~mm}$ of graphite exposed to the plasma. Also soldered to the brass connector is an $11 \mathrm{nF}$ floating capacitor. This capacitor shorts high frequency plasma fluctuations to allow the probe tip sheath to follow the rf potential. Both the probe tip and capacitor are then fit through a Macor cap, which shields the internal vacuum side circuitry from the plasma as well as adds structural support to the probe tip. Another Macor cap is then fit over the exposed capacitor leg so that only the probe tip and alumina jacket are interacting with the plasma. The atmosphere side of the assembly is shown in Fig. 3.9 and mounted to REVAN such that it can translate along $\hat{z}$.

The probe assembly is attached to the to chamber by a custom welded flange, replacing the front window of REVAN with a $\varnothing 4.5 "$ port in the center of an aluminum blank (see Fig. 3.9). This allows for one-dimensional movement in the $\hat{z}$-direction and is aligned with the center of the extraction optics and wafer. The probe tip 


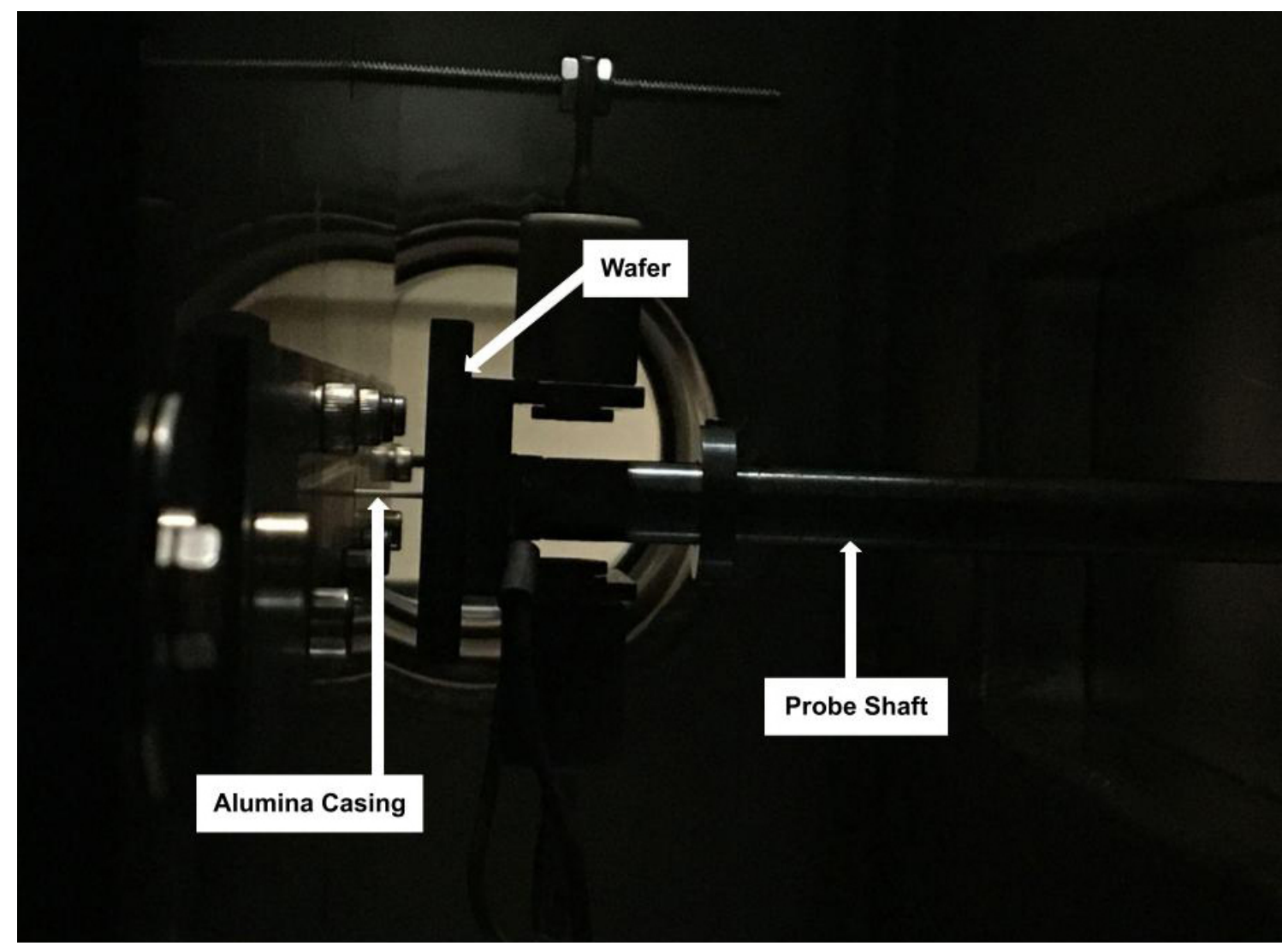

Figure 3.10: The view inside the vacuum chamber from a $\varnothing 8 "$ side port. The Langmuir probe tip passing through the wafer and aperture is shown.

extends a distance $25 \mathrm{~mm}$ from the Macor cap to maximize measurement range in the $-\hat{z}$-direction. Measurements are limited to $z=-10 \mathrm{~mm}$ due to the Macor contacting the biased wafer. Figure 3.10 shows the vacuum side of the assembly with the probe at the maximum $z$-position. The probe is zeroed in the coordinate system by visually aligning the tip with the face of the extraction optics. An external scale is used for translation reference. This gives the probe a position uncertainty of $\delta z= \pm 1.03 \mathrm{~mm}$.

\subsubsection{Electronics}

The EM waves in an ICP create an oscillating potential in the plasma and across the sheath, which affects the I-V trace collected by the Langmuir probe [119]. This effect is minimized by modifying the probe circuitry with rf-chokes [120]. By implementing specific self-resonant inductors in the signal path, large impedance values will block 
fluctuations at their corresponding rf frequencies. To achieve full coverage, inductors at the full, half, and double resonant frequencies of $13.56 \mathrm{MHz}$ were soldered into the Langmuir probe circuit. Figure 3.11 shows the electrical diagram of the probe circuit.

Lenox-Fugle International, Inc. inductors were used with self-resonant frequencies of $13.2 \mathrm{MHz}, 6.8 \mathrm{MHz}$, and $26 \mathrm{MHz}$. An Agilent 33220A Arbitrary Waveform Generator was used to confirm the signal reduction at these frequencies. The waveform generator produced a $13.56 \mathrm{MHz}$ signal which was filtered through the Langmuir probe and was compared against an unfiltered signal with a Textronics Oscilloscope. Figure 3.12 shows the complete attenuation of a $13.56 \mathrm{MHz}$ signal, which was also confirmed at 27.1 MHz and 6.78 MHz.

\subsubsection{Analysis}

\subsubsection{I-V Trace}

The Langmuir probe is biased with a Keithly 2400 SourceMeter, which also records the collected current. The bias probe potential $V_{B}$ is swept from negative to positive

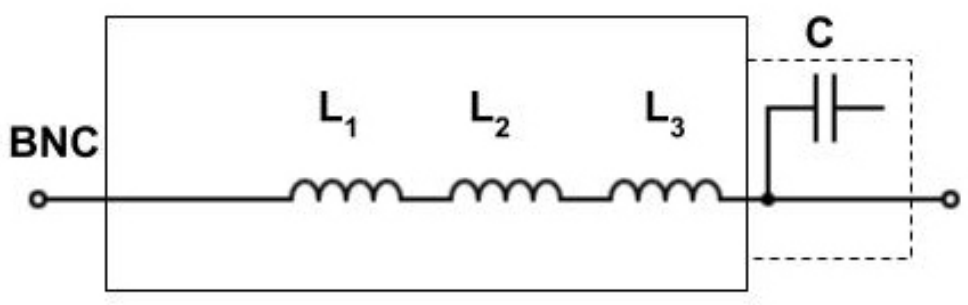

Figure 3.11: An electrical diagram of the Langmuir probe circuit. The inductors have a self resonance at $\mathrm{L}_{1}=6.8 \mathrm{MHz}, \mathrm{L}_{2}=13.56 \mathrm{MHz}$, and $\mathrm{L}_{3}=26 \mathrm{MHz}$. To short high frequency plasma fluctuations, an $11 \mathrm{nF}$ floating capacitor $\mathrm{C}$ is soldered in parallel with the probe tip. The Macor cap is denoted by the dashed line. 


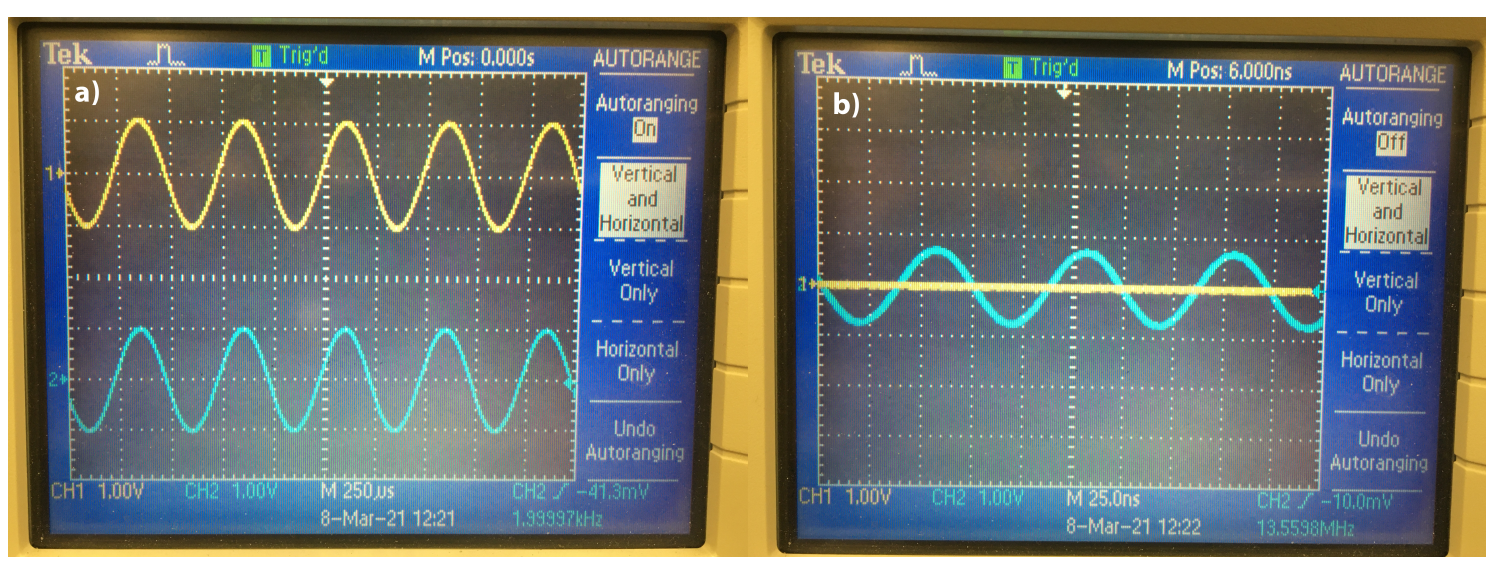

Figure 3.12: a) Shows the sourced $13.56 \mathrm{MHz}$ signal (blue) and the result of the signal passing through an unfiltered Langmuir probe (yellow). b) Shows the signal at $13.56 \mathrm{MHz}$ with the rf-choke in place and is compared source signal. As seen, the rf-choke can efficiently filter the antenna frequency of $13.56 \mathrm{MHz}$.

values to produce an I-V trace, shown in Fig 3.13. The probe only collects particles that have sufficient energy to overcome the applied bias to the probe tip. There are three distinct regions in the I-V trace [121]:

- Ion Saturation Region: When the probe is biased very negatively, few electrons have the energy needed to overcome the bias. Therefore, the current collected in this region is from ions. If the bias is sufficiently negative, then the ion current will saturate, giving an approximately constant current value. This region is defined as when the bias potential is much less than the floating potential $\left(V_{B} \ll V_{f}\right)$

- Transition Region: Between the floating potential $V_{f}$ and the plasma potential $V_{p}$ is considered the transition region $\left(V_{f}<V_{B}<V_{p}\right)$. In this bias range, both ions and energetic electrons will contribute to the probe's current. If the electrons in the plasma are Maxwellian, then the electron current will increase exponentially with increasing voltage in this region.

- Electron Saturation Region: When the probe reaches high positive biases $\left(V_{B} \gg V_{p}\right)$ the total current will be approximately constant. The probe will collect all electrons that enter its sheath and repel ions. 


\subsubsection{Fluid Quantities}

Given that REVAN has no magnetic fields in the measurement region, the conventional method [29], also known as the non-drifting Maxwellian electrons method [122], is used to calculate $n_{e}$ and $T_{e}$. All measurements with the Langmuir probe were taken with a wafer bias of $0 \mathrm{~V}$. This was to prevent arcing from the wafer to the probe. Without this bias there is no unipolar sheath and electrons are collected in all regions.

The total current $I_{\text {total }}$ collected by the probe tip is the net flux of both impinging ions and electrons such that

$$
I_{\text {total }}=I_{e}-I_{i}
$$

where $I_{e}$ is the electron current and $I_{i}$ is the ion current. The transition region yields the most relevant quantities, typically with significant noise. These random fluctuations are exacerbated when taking a derivative of the signal. To minimize the uncertainty associated with the inherent noise, a smoothing spline is fit to each trace before processing.

The ion saturation current is calculated by fitting a line to the region of the trace with very negative bias and extrapolating a linear fit across the sweep. This step also introduces some uncertainty as the ion saturation region is dependent on sheath expansion for cylindrical probe tips. As the bias becomes more negative, the ion collection sheath expands, allowing for an increased flux of ions to the probe tip. This manifests itself as a linearly decreasing current in the I-V trace. After the ion current is determined, it is added to the total current from Eqn. 3.12 to isolate the electron current.

Once $I_{e}$ is determined, several plasma parameters are extracted from the I-V sweep. Here, the plasma potential is defined as the inflection point of the electron 


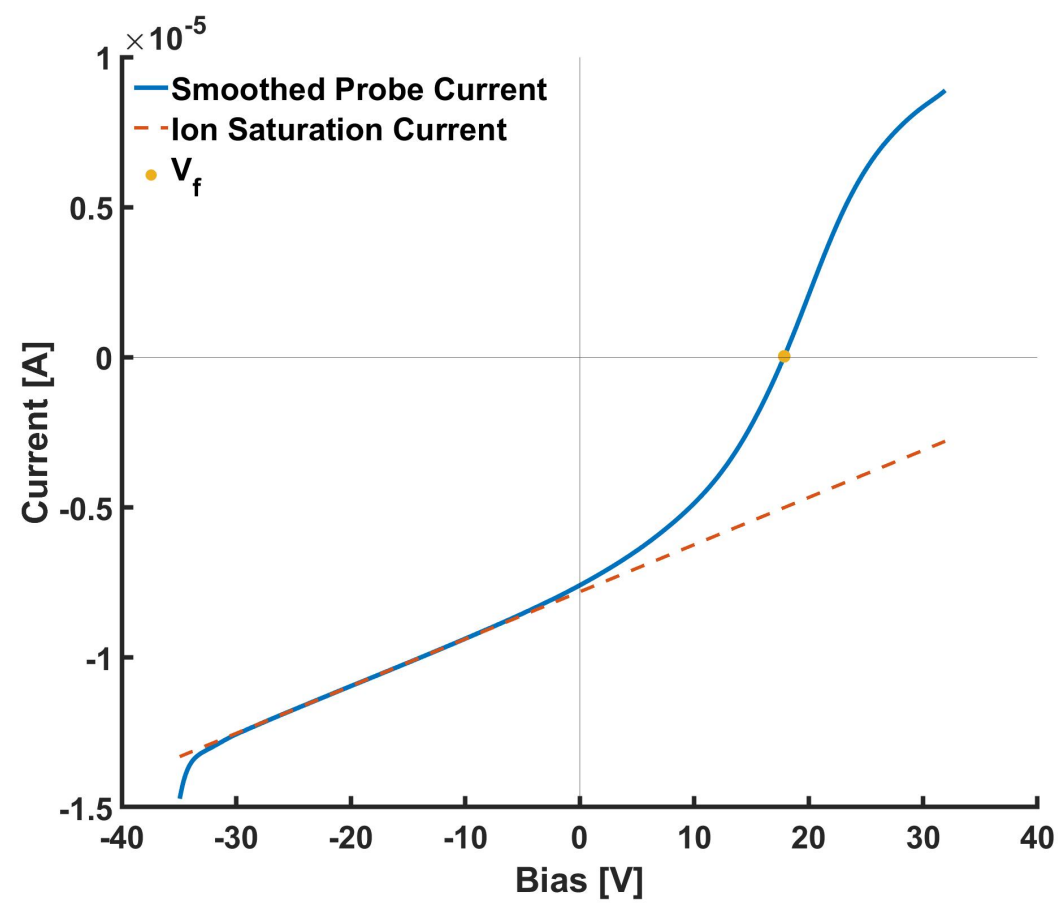

Figure 3.13: An example I-V trace obtained in a REVAN plasma. The collected current (solid) was produced by a spline fit. The ion saturation current is fit to a straight (dashed) line and the floating potential $\mathrm{V}_{\mathrm{f}}$ is shown. At very negative biases $\left(V_{B}<30 \mathrm{~V}\right)$ arcing occurred. This results in a sudden decrease in current which is not part of $I_{\text {sat }}$ and is not considered when determining the ion saturation current.

current. This is obtained by taking the first derivative of $I_{e}$ and calculating the maximum of the derivative. The floating potential is the value at which the total current crosses $I_{\text {total }}=0$.

The electron distribution function $f$, assuming a non-drifting Maxwellian, has a form of $[122]$

$$
f(\vec{x}, \vec{v}, t)=n_{e}\left(\frac{m_{e}}{2 \pi T_{e}}\right)^{3 / 2} \exp \left[-\frac{m_{e} v^{2}}{2 T_{e}}\right]
$$

This is related to the current density $j_{e}$ to the probe through

$$
\begin{aligned}
j_{e}\left(V_{B}\right) & =e \int f(\vec{x}, \vec{v}, t) \vec{v}_{z} \cdot \hat{n} \quad d^{3} v \\
& =e n_{e}\left(\frac{m_{e}}{2 \pi T_{e}}\right)^{3 / 2} \int_{v_{\text {min }}}^{\infty} \exp \left[-\frac{m_{e} v^{2}}{2 T_{e}}\right] \vec{v}_{z} \quad d v_{z}
\end{aligned}
$$

where $\hat{n}$ is the direction normal to the probe's surface. Integrating Eqn. 3.15, 
and multiplying by the probe tip's area $A_{p}$, an expression for the probe current is obtained,

$$
\begin{array}{rlrl}
I_{e}\left(V_{B}\right) & =I_{e}^{*} \exp \left[\frac{-e\left(V_{p}-V_{B}\right)}{T_{e}}\right], & & V_{B} \leq V_{p} \\
I_{e} & =I_{e}^{*}, & V_{B}>V_{p} .
\end{array}
$$

Here, $I_{e}^{*}$ is the electron saturation current. Equation 3.16 is a relation between the electron current and the electron temperature. Taking the derivative of the natural $\log$ of Eqn. 3.16, the electron temperature is rewritten as

$$
T_{e}=\left[\frac{d \ln I_{e}\left(V_{B}\right)}{d V}\right]^{-1}
$$

Therefore, the electron temperature is the inverse slope fit to a semi-log plot of the derivative of the electron current. An example of the fit, from which the electron temperature for a given electron energy probability function (EEPF) was calculated, is shown in Fig. 3.14.

For a Maxwellian distribution, the electron saturation current has the form of

$$
I_{e}^{s a t}=\frac{n_{e} e A_{p}}{4} \sqrt{\frac{8 k_{B} T_{e}}{\pi m_{e}}} .
$$

Therefore, the electron density is calculated from Eqn. 3.19:

$$
n_{e}=\sqrt{\frac{2 \pi m_{e}}{k_{B} T_{e}}} \frac{I_{e}^{s a t}}{e A_{p}}
$$

if the electron saturation current is known.

Determination of the electron saturation current, however, introduces its own uncertainties. The electron saturation current is the current at the plasma potential. The analysis carried out here identifies the plasma potential as the inflection point of the $\mathrm{I}-\mathrm{V}$ trace as done in References $[110,123]$. Alternatively, the plasma 

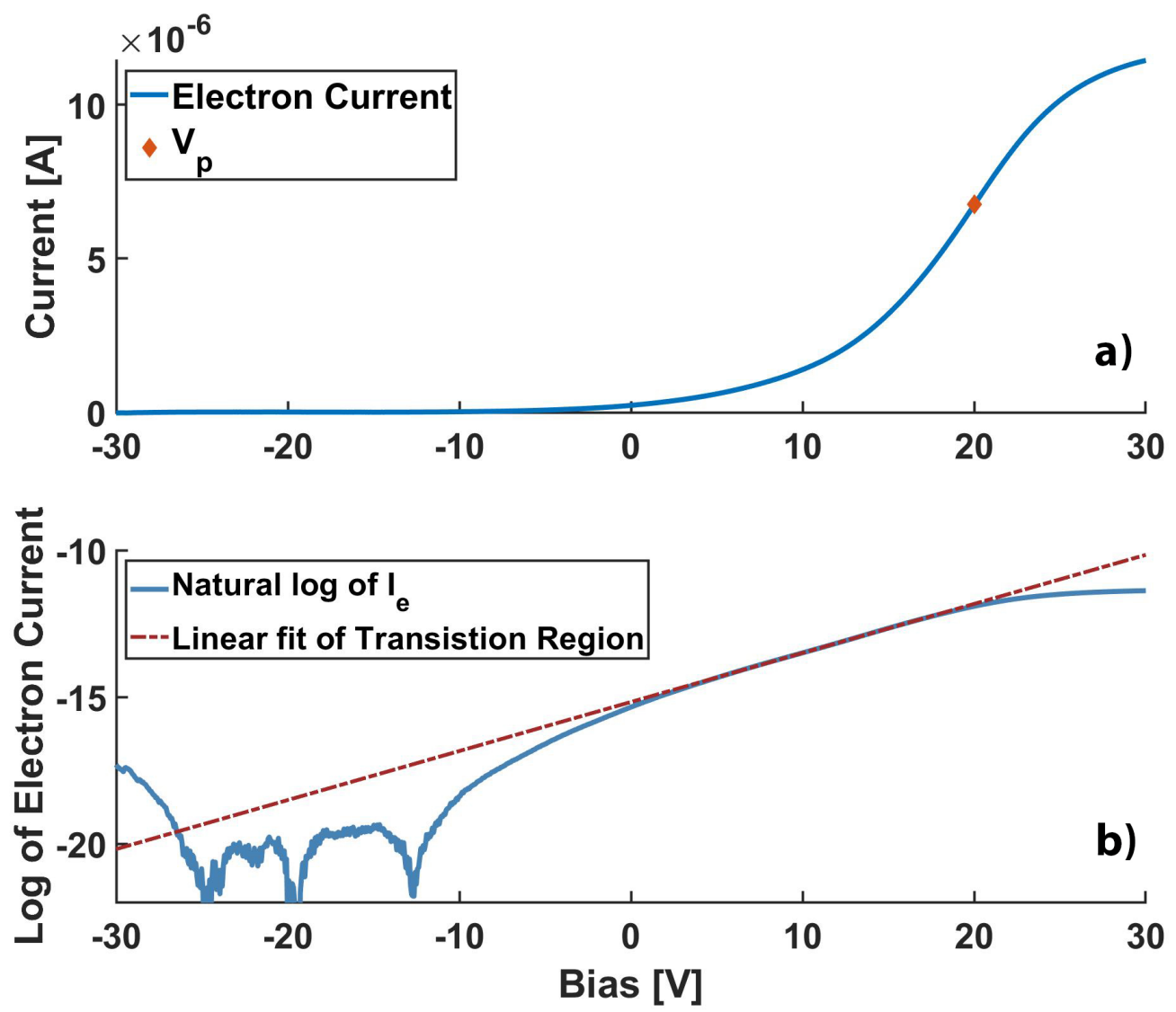

Figure 3.14: a) The isolated electron current from an I-V trace is shown, as well as the inflection point (diamond). b) Is the semi-log of $I_{e}$ with a linear fit applied to the transition region of the trace for temperature calculation.

potential can be taken at the "knee" of the I-V curve and is found by the intersection of extrapolated transition region and electron saturation region fits [122]. Adding further uncertainty to the plasma potential is the fact that it is in an exponential region, so a change of a few volts can lead to large changes in measured current. Using these two methods, the electron saturation current typically carries an uncertainty of $\delta I_{e} \approx 20 \%$ [83].

The electron saturation current was used to calculate plasma density, instead of the ion saturation current, because of the non-classical shape of the I-V traces inside REVAN. A comparison of I-V traces obtained inside and outside of the ion source are shown in Fig. 3.15. The steadily decreasing total current in the ion saturation region, for measurements inside the plasma source, makes it impossible to get a density value from the ion saturation current. This shape is not from contamination 
because the typical I-V trace was recovered outside the source after all measurements were performed. One possibility is the presence of fast electrons [124]. This is unlikely though as there is no applied potential to create an electron beam.

Another possibility is that the probe is experiencing a rectification current due to the rf field. Rf rectification occurs when an rf potential across a sheath produces a time-averaged change in the sheath's current and/or voltage [125]. The sheath can respond by an increase in the plasma potential to cancel the increased electron current. This explains the high $V_{p}$ seen in the I-V trace and is more probable than an electron beam in REVAN. Additionally, the discharge during Langmuir probe measurements was operating in E-mode (see Appendix A) and therefore the antenna's potential is not attenuated inside the ion source.

\subsection{Gaussmeter}

REVAN has a magnetic cusp field in the ion source to improve plasma uniformity. Cusp fields are not uncommon in these sources as they extend operational lifetime of the source. However, introducing magnetic fields will severely change the ion and electron dynamics. The strong potential applied to the wafer gives rise to the
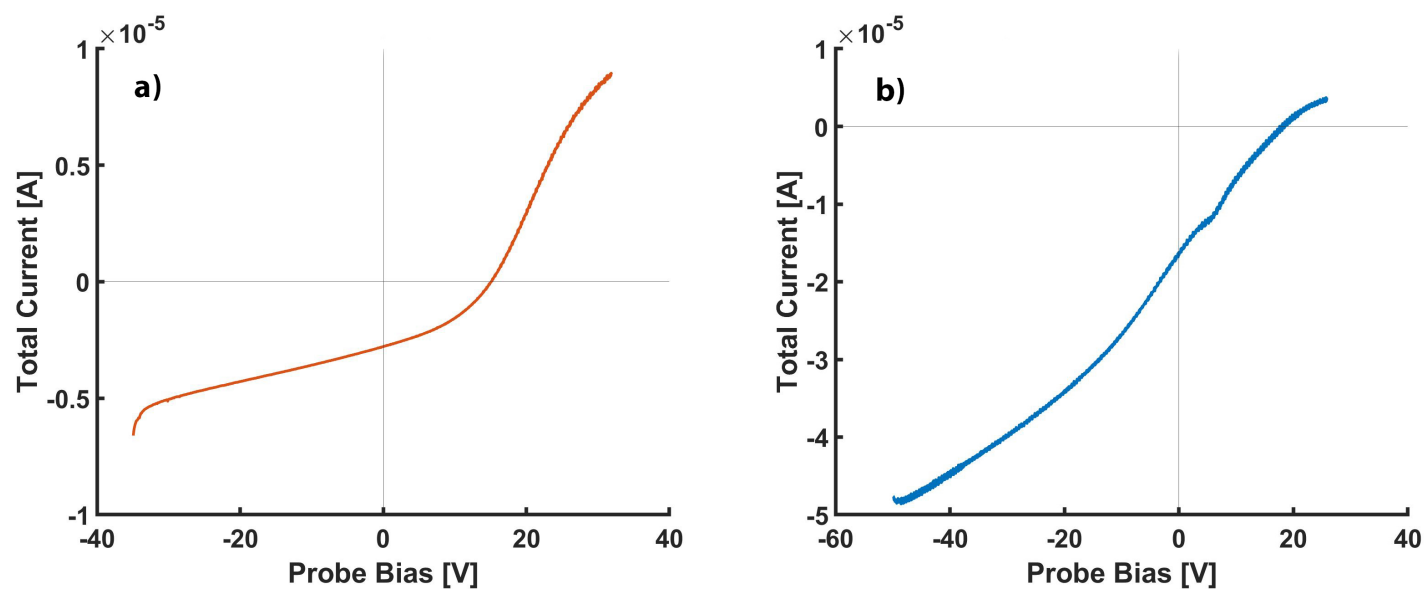

Figure 3.15: A comparison of I-V traces obtained a) outside the ion source and b) inside the ion source. The non-classical shape inside the ion source is most likely due to an rf sheath rectification. 
possibility of an $\vec{E} \times \vec{B}$ drift in the system. To confirm that the extracted ions are not subject to any additional forces, the magnetic field was confirmed to be negligible on the axis of the beam.

Magnetic fields in REVAN were measured with a Lake Shore gaussmeter probe (455 DSP). This gaussmeter has an operational range of $\mathrm{mG}$ up to $\mathrm{kG}$ with $0.02 \mathrm{mG}$ resolution and $\pm 0.075 \mathrm{mG}$ accuracy [126]. The sensor is a rectangular InAs or GaAs semiconductor in the probe tip that is supplied with a current from the gaussmeter. When the sensor is in a magnetic field, the semiconductor's electrons feel a Lorentz force. This force causes the electrons to drift orthogonally to both the current and magnetic field.

This creates a charge separation in the semiconductor, the build up of which results in an internal electric field,

$$
\vec{E}_{G}=-\frac{1}{n e} \vec{J} \times \vec{B}
$$

This electric field has an associated electric potential between two sides of the detector. The electric potential is related to magnetic field strength by

$$
V_{s}=\gamma_{B} B \sin \theta
$$

where $V_{s}$ is the potential across the sensor, $\gamma_{B}$ is a proportionality constant, $B$ is the magnetic field in $\mathrm{G}$, and $\theta$ is the angle between the magnetic field and the normal of the sensor.

The probe tips, shown in Fig. 3.16, have a configuration such that only the magnetic field along the axis of the probe is measured, or fields normal to the sensor's face. The gaussmeter is calibrated using a $\mu$-metal jacket to zero the circuits. Magnetic field measurements are displayed by digital read-out of the circuits and recorded by hand. Measurements confirmed that the cusp field is created by rareearth magnets of $10000 \mathrm{G}$ strength. The strength of the magnetic field decreases 


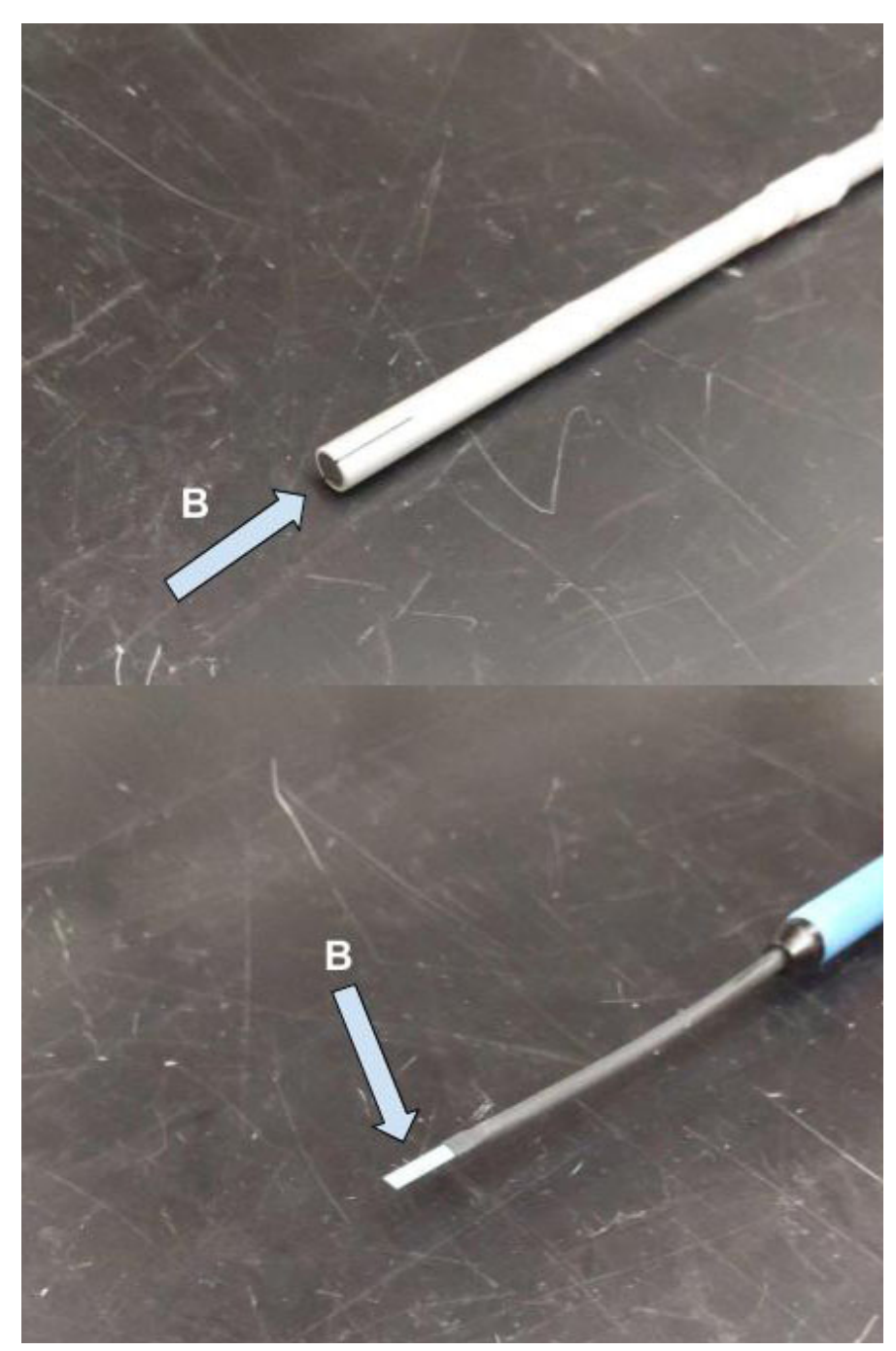

Figure 3.16: The two types of Gaussmeter configurations. The top image is used for axial field measurements while the bottom image is used for transverse fields measurements.

rapidly with distance, producing a field of $5.79 \mathrm{G}$ at the aperture and $0.49 \mathrm{G}$ at the wafer. At the farthest measurable distance inside the source $(z=-25)$ the field was 33.17 G. 


\section{Chapter 4}

\section{Results}

\subsection{Introduction}

Measurements were made of the ion velocity distribution function (IVDF) both in the beam and in the ion source for different extraction geometries. Depending on the extraction aperture, potential, and source properties the extracted ion beam will have a different focal point due to the curvature of the plasma meniscus boundary. This boundary is formed by the balance between the Debye sheath formed inside the ion source and the unipolar sheath formed by the extraction potential. The aim of this investigation was to quantify how controllable source properties affect the formation and trajectories of extracted ion beams.

\subsection{Angled ion beam from a slit}

Initial experiments in REVAN were conducted to investigate whether LIF was capable of measuring extracted ion beams as well as resolving a beam angle. The slit optics (Sections 2.4.2.1 - 2.4.2.2) were used to produce an angled ion beam shown in Fig. 4.1. For these measurements, there was no extraction potential since the ion 
current shorted any applied bias. Therefore, a meniscus is not formed and the ions diffuse out of the slit according to the Debye sheath. The ions exit the ion source through a $5 \mathrm{~mm}$ gap, which makes the formation of a continuous Debye sheath possible. However, due to the restricted optical access of the slit optics, confocal measurements of the meniscus could not be performed.

Measurements were taken outside of the source with the 2D optical arrangement for a constant power of $P_{f}=4 \mathrm{~kW}$ in both the $\hat{y}$ and $\hat{z}$ directions. Due to the large extraction area for the slit optics, the ion signal was low in the extracted region. This limited the rf power for ion measurement to $P_{f}=4 \mathrm{~kW}$ because lower power would not produce enough metastables for measurement and at higher powers the rf

Ion Source

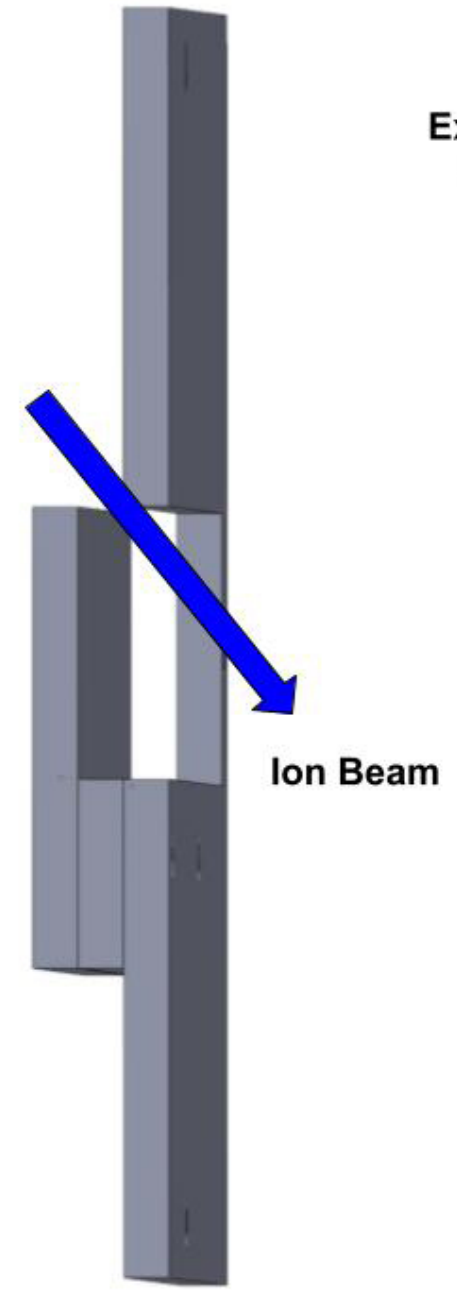

Extraction

region

Figure 4.1: Diagram of the slit extraction optics and the angled flow of ions through the slit. 


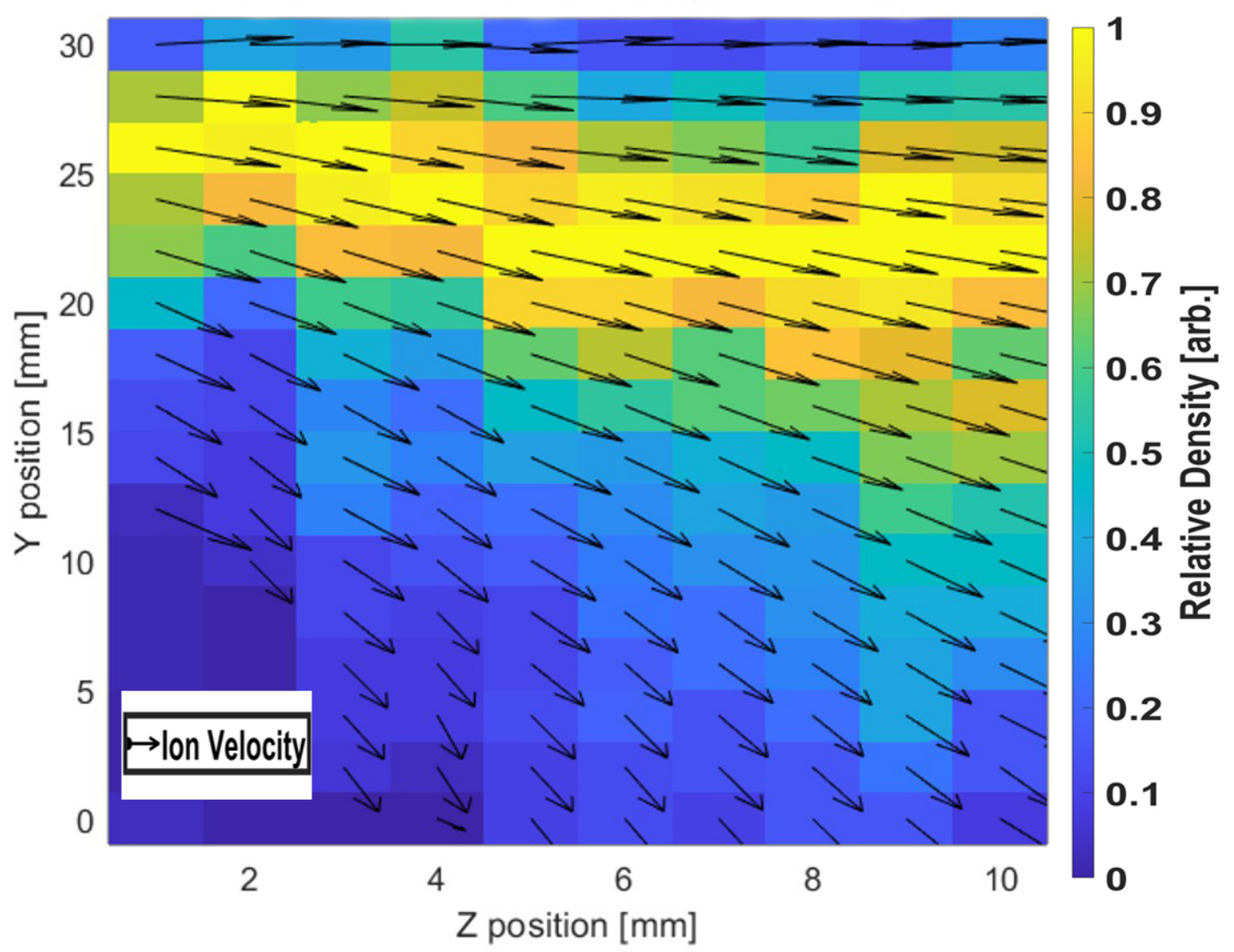

Figure 4.2: The ion beam velocity and relative metastable density as a result of the slit optics. The density is shown in the colormap and is normalized to one. The magnitude of the arrows are scaled such that the largest arrow is equal to $9.5 \mathrm{~km} / \mathrm{s}$. A divergent beam is clearly evident in both velocity and density.

generator would overheat. The IVDFs obtained with this configuration had a nonMaxwellian lineshape so the moments of the distribution (Eqns. 3.3 - 3.4) were used to calculate relative ion density and velocity. Multiple, repeated shots were taken at a fiducial location outside the source. These measurements provide statistics on the reproducibility of the plasma as well as the experimental uncertainty inherent in the $2 \mathrm{D}$ optical arrangement measurements and give an uncertainty in metastable density $\delta n_{i, 2 D}<1 \%$. The wavemeter has a velocity uncertainty of $\delta v_{W . M .}= \pm 93$ $\mathrm{m} / \mathrm{s}$, which when combined with the standard deviation in velocity gives a total velocity uncertainty of $\delta v_{2 D} \approx \pm 125 \mathrm{~m} / \mathrm{s}$.

In this coordinate system, $z=0 \mathrm{~mm}$ is the front of the extraction optics, $x=0$ $\mathrm{mm}$ is the center of the ion source, and $y=0 \mathrm{~mm}$ is the bottom of the extraction 
slit. Data were obtained at $1 \mathrm{~mm}$ increments from $z=0$ to $z=10 \mathrm{~mm}$, while measurements in $y$ were obtained at $2 \mathrm{~mm}$ increments between $y=0 \mathrm{~mm}$ and $y=30 \mathrm{~mm}$. All measurements were taken in the $x=0 \mathrm{~mm}$ plane. Figure 4.2 shows the ion velocity overlaid with the relative metastable density for an ion beam produced by the slit optics. The relative metastable density was calculated from the average of the $\hat{z}$ and $\hat{y}$ contributions at each location and then normalized to one. The arrows show the ion velocity at each measurement location, and their magnitudes are scaled such that the largest arrow represents a velocity of $9.5 \mathrm{~km} / \mathrm{s}$. Ion signal in was too low at $z<3 \mathrm{~mm}$ and $y<10 \mathrm{~mm}$ for velocity to be calculated.

From the measurements in both density and velocity, there is a clear beam structure that is diverging. It starts centered at $y=26 \mathrm{~mm}$ and with a width of $\approx 8 \mathrm{~mm}$. As this beam propagates through the vacuum chamber, the beam spreads out according to the velocity flow field, with little to no density increase above $y=26 \mathrm{~mm}$. Most of the beam density spreads out to lower $y$ values, as expected for a beam generated by these extraction optics. By the end of the measurement region, the beam has expanded to $\approx 14 \mathrm{~mm}$. The angle of this beam is found by locating the peak density at $z=0 \mathrm{~mm}$ and $z=10 \mathrm{~mm}$ and using trigonometric relations to obtain a beam angle of $\approx-30^{\circ}$ with respect to $\hat{z}$. This angle is not as large as expected given the sharp angle of extraction from the optics. However, given the large ion velocity in $\hat{z}$ this shallow beam angle is not unreasonable. The ions in the source must already have a large $\hat{z}$ velocity when they interact with the Debye sheath, which imparts a relatively small velocity in the $-\hat{y}$-direction, resulting in a shallow beam angle. While these measurements proved that LIF can measure angular beam properties for a semiconductor processing ion beam, the configuration did not provide the level of control necessary to investigate the ion dependencies on source parameters. Therefore the rest of the work was performed with the aperture optics. 


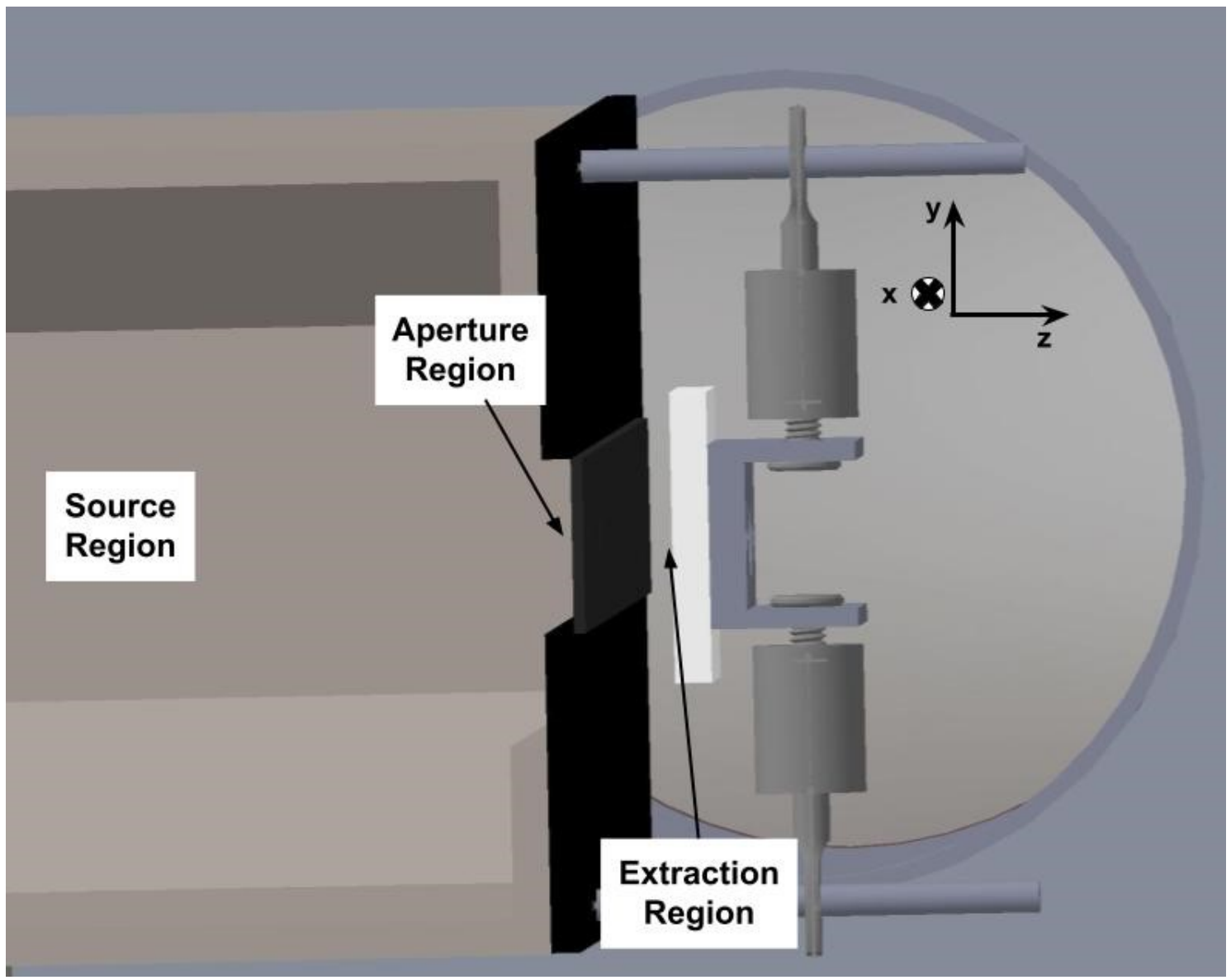

Figure 4.3: View of the REVAN chamber and the three areas investigated: the source region, the aperture region, and the extraction region.

\subsection{Ion beam extracted from a plasma meniscus}

REVAN has three distinct regions in which measurements were performed. Deep in the source, $z \leq-10 \mathrm{~mm}$, where electrons are affected by the induction field is called the source region. The aperture region, $-5 \leq z \leq 1 \mathrm{~mm}$, is where the particles encounter the Debye sheath and are accelerated according to the sheath's potential structure. The topology of the potential in this region changes depending on source parameters, governing downstream ion properties. Lastly, the region outside of the source, $z \geq 1 \mathrm{~mm}$, where an ion beam forms in a unipolar sheath and is accelerated through the vacuum chamber to the negatively biased wafer, is called the extraction region.

Because the extraction area of the aperture optics was much smaller than the slit 
optics, there was a higher ion signal in the beam, allowing for measurements at $P_{f}=$ $1,2,3$, and $4 \mathrm{~kW}$ of $\mathrm{rf}$ power. Additionally, the biased wafer was maintained at a steady potential since the extracted ion current was lower in this configuration. This allows for a more controllable ion beam because source parameters and bias potential now affect the plasma meniscus structure. Therefore, the source properties' effect on the extracted ion beam was investigated for the aperture optics configuration.

Due to source degradation, H-mode operation was not possible for measurements outside of the source. These extraction region data were obtained towards the end of the experimental campaign and disagreement between early and late fiducial measurements indicated that the plasma source was no longer inductively coupled. Attempts to restore REVAN to operate in H-mode were unsuccessful, so for these measurements the source was operating in E-mode. A discussion of troubleshooting methods attempted is given in Appendix A.

Ion properties were measured along the path of the extracted ions for $z>0$ $\mathrm{mm}$. These measurements were taken with the $2 \mathrm{D}$ stage along the axis of the beam. Both the $\hat{z}$ and $\hat{y}$ components were measured at three locations along the beam $(z=1,7,12 \mathrm{~mm})$. While these measurements were taken in an E-mode plasma, the sheath theory previously discussed in Section 1.3.1 is still true. Therefore, the focal point should be dependent on the meniscus shape with more concave menisci producing shorter focal points and convex menisci producing divergent beams. The major difference in these measurements is that the ion density in the source is lower. This prevents these measurements from being directly comparable to the data reported later in Section 4.5, but nevertheless provide important insights into the properties of extracted beams.

Two potentials, $0 \mathrm{~V}$ and $3000 \mathrm{~V}$, were compared for all input powers. In the case with no bias, Fig. 4.4 shows the resulting ion motion through the Debye sheath without an extraction potential. The beam is only dependent on the rf-input, which dictates the length of the Debye sheath. Since there is no unipolar sheath without 


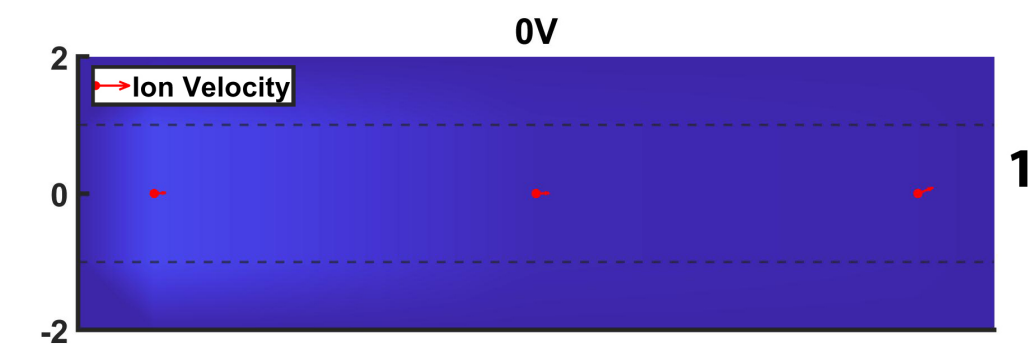

\section{$1 \mathrm{~kW}$}

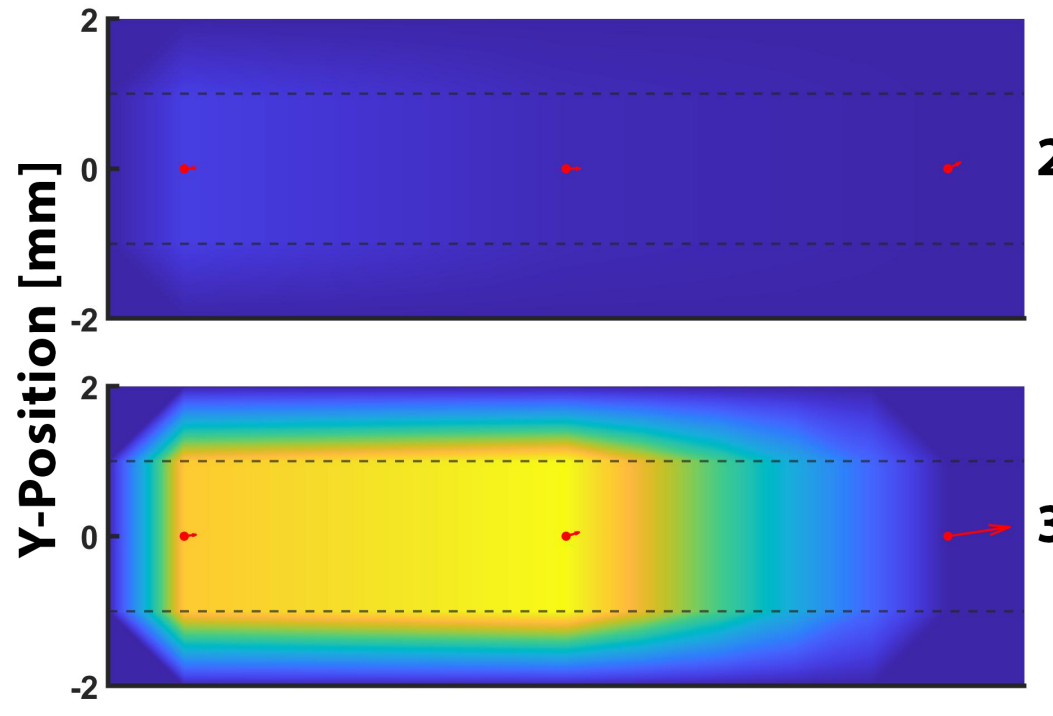

\section{$2 \mathrm{~kW}$}

\section{$3 \mathbf{k W}$}

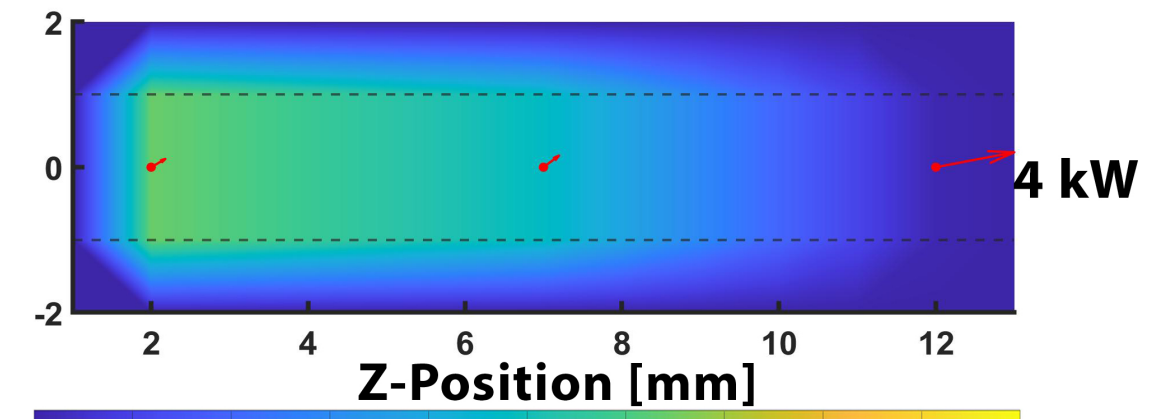

0

Relative Density [arb.]

Figure 4.4: Measurements of the ion population outside of the source for no extraction potential. The metastable density of the ions is shown by the colormap and has been normalized to the largest density signal across all powers. The red arrows show the velocity of ions. The arrows' magnitudes have been scaled so that the largest is equal to the maximum speed of $12,000 \mathrm{~m} / \mathrm{s}$.

an extraction potential to form a convex meniscus, all beams are divergent. At $P_{f}=1 \mathrm{~kW}$ and $P_{f}=2 \mathrm{~kW}$ the ion signal is relatively low. For $P_{f}=3 \mathrm{~kW}$, the relative density signal persists for $z=2-7 \mathrm{~mm}$. This indicates that the beam is overall divergent, but has good collimation up to $z=7 \mathrm{~mm}$. At $P_{f}=4 \mathrm{~kW}$ the peak density is at $z=0$ and drops at further measurement points. This shows that at higher powers the Debye sheath produces beams with a higher degree of divergence 


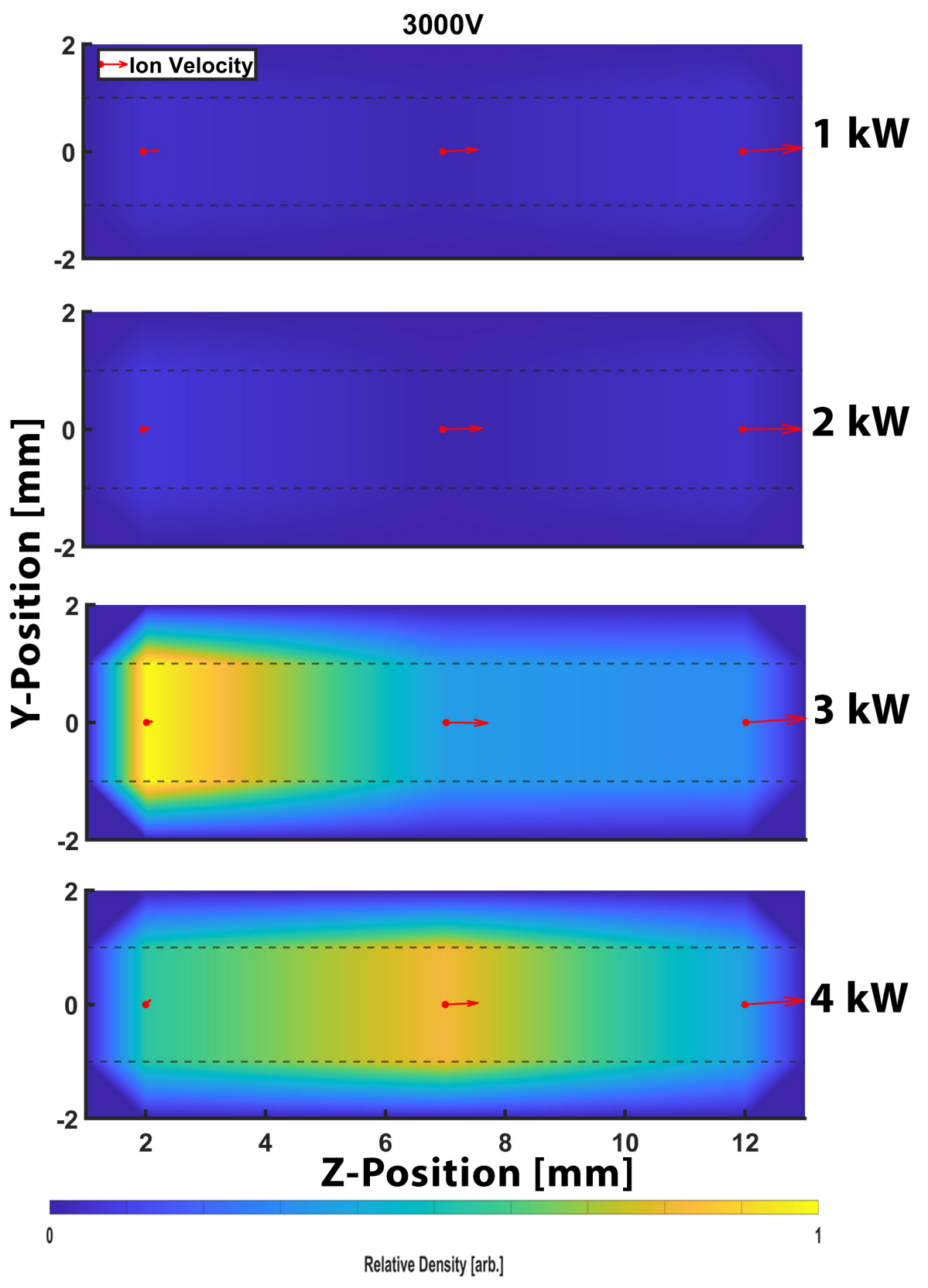

Figure 4.5: Measurements of the ion population outside of the source for a $3000 \mathrm{~V}$ extraction potential. The metastable density of the ions is shown by the colormap and has been normalized to the largest density signal across all powers. The red arrows show the velocity of ions. The arrows' magnitudes have been scaled so that the largest is equal to the maximum speed of $105,000 \mathrm{~m} / \mathrm{s}$.

where most of the ions diverging rapidly after $z=0$ for $P_{f}=4 \mathrm{~kW}$. At $P_{f}=3 \mathrm{~kW}$ the the beam is more collimated, losing signal due to divergence after $z=7 \mathrm{~mm}$.

In Fig. 4.5 the wafer is biased to $3000 \mathrm{~V}$ and the plasma meniscus is balanced by a constant unipolar sheath and a Debye sheath. $P_{f}=1 \mathrm{~kW}$ and $P_{f}=2 \mathrm{~kW}$ have relatively low density signals making the beam difficult to detect, similar to 
Fig. 4.4. At $3 \mathrm{~kW}$ the relative density suggests a focal point close to the aperture, while $4 \mathrm{~kW}$ has a focal point closer to $z=7 \mathrm{~mm}$. Comparing Fig. 4.4 to Fig. 4.5, the data follows the predictions for the focusing of an ion beam due to curvature changes in the plasma meniscus. Since the Debye sheath more strongly opposes the unipolar sheath for $P_{f}=4 \mathrm{~kW}$ compared to $P_{f}=3 \mathrm{~kW}$, the radius of curvature is greater and the focal spot is further from the source at larger rf power.

In an ideal laminar beam, the particles flow in layers that never intersect. Therefore, particles at the same radial position must have the same transverse velocity and the magnitude of the transverse velocity is linearly proportional to the distance from the axis of the beam (Fig. 4.6). Assuming a laminar beam, the focal properties of the beam are determined from the velocity in the transverse direction [115]. If the

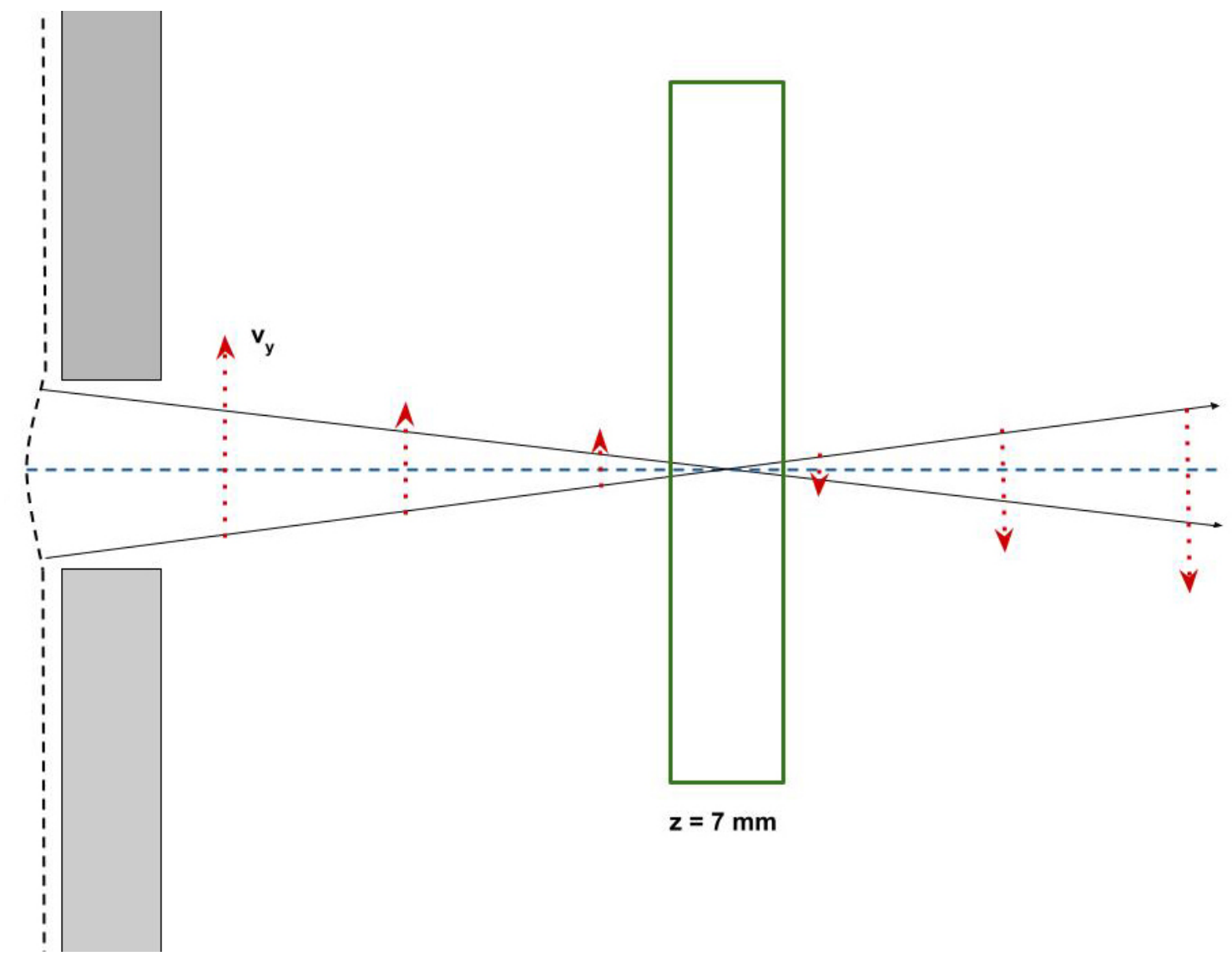

Figure 4.6: Diagram of the transverse ion velocity along the path of extraction for a focused beam. For a laminar beam, the transverse velocity (red arrows) will decrease until the focal plane is reached, after which the velocity will increase. An approximate $z=7 \mathrm{~mm}$ location is designated in order to put the results at $4 \mathrm{~kW}$ into context. 
transverse velocity $v_{y}$ decreases with increasing distance, then the beam is coming to a focal point, while if the velocity is increasing with distance then the beam is divergent. The values of ion temperature and velocity in $\hat{y}$ and $\hat{z}$ for $0 \mathrm{~V}$ and 3000 V applied biases are given in Fig. 4.7 and Fig. 4.8, respectively. In Fig. 4.7 the ion velocity in $\hat{z}$ is mostly uniform, with an average velocity of $\approx 10,000 \mathrm{~m} / \mathrm{s}$. The ion temperature in both directions is room-temperature at $\approx 0.025 \mathrm{eV}$. Due to the large amount of noise at lower powers, $P_{f}=2 \mathrm{~kW}$ has an artificially large temperature and low velocity. In the case of $0 \mathrm{~V}$, all rf powers, except $P_{f}=2 \mathrm{~kW}$, produce a positive slope in $v_{y}$ throughout the measurement region. As mentioned before,
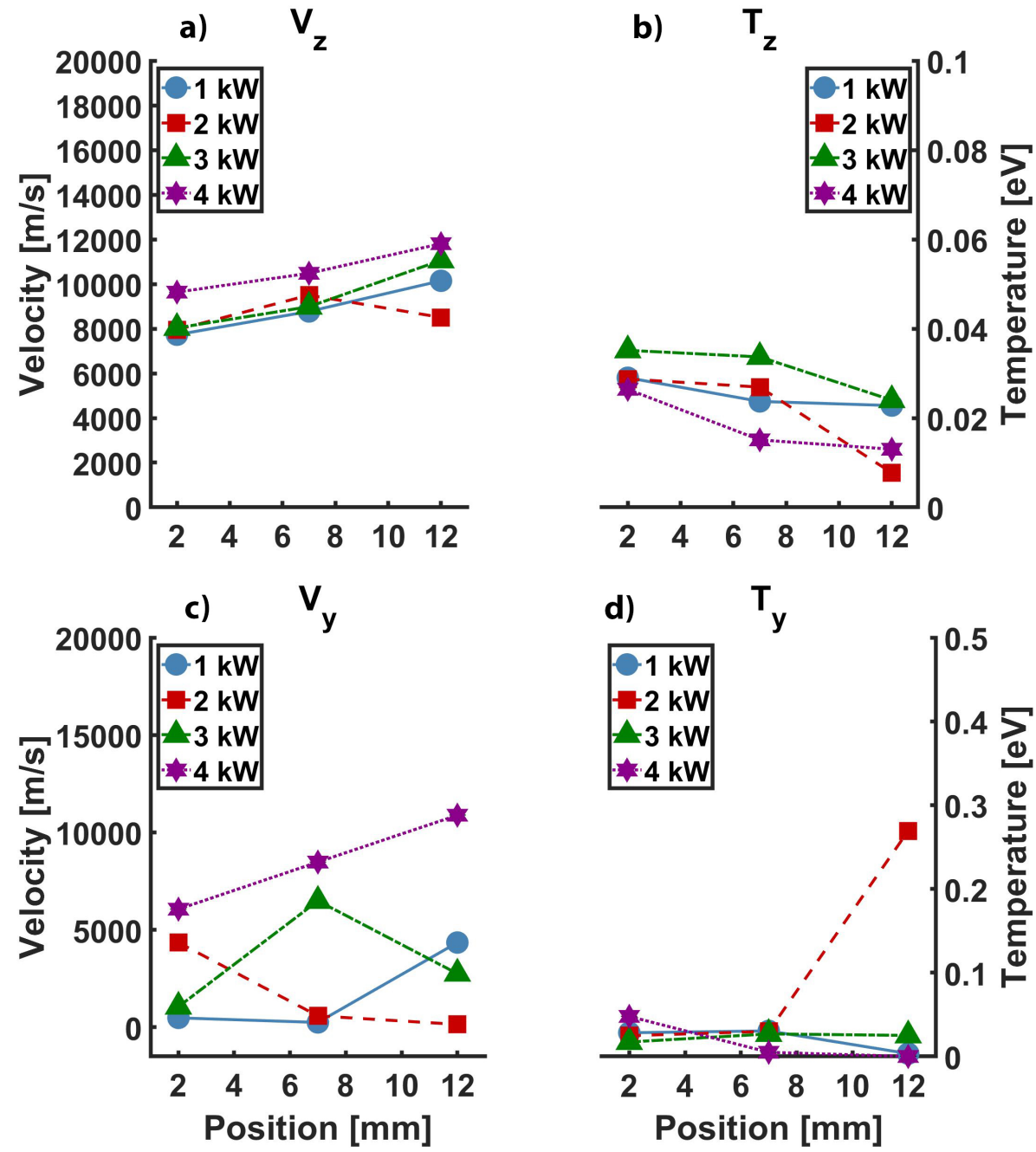

Figure 4.7: The velocity and temperature calculated for IVDFs outside the source in both $\hat{z}$-and $\hat{y}$-directions with no extraction bias. The ion temperature is close to room temperature for both components. The positive slopes for $P_{f}=3$ and $4 \mathrm{~kW}$ in $v_{y}$ indicate the beam is diverging. 

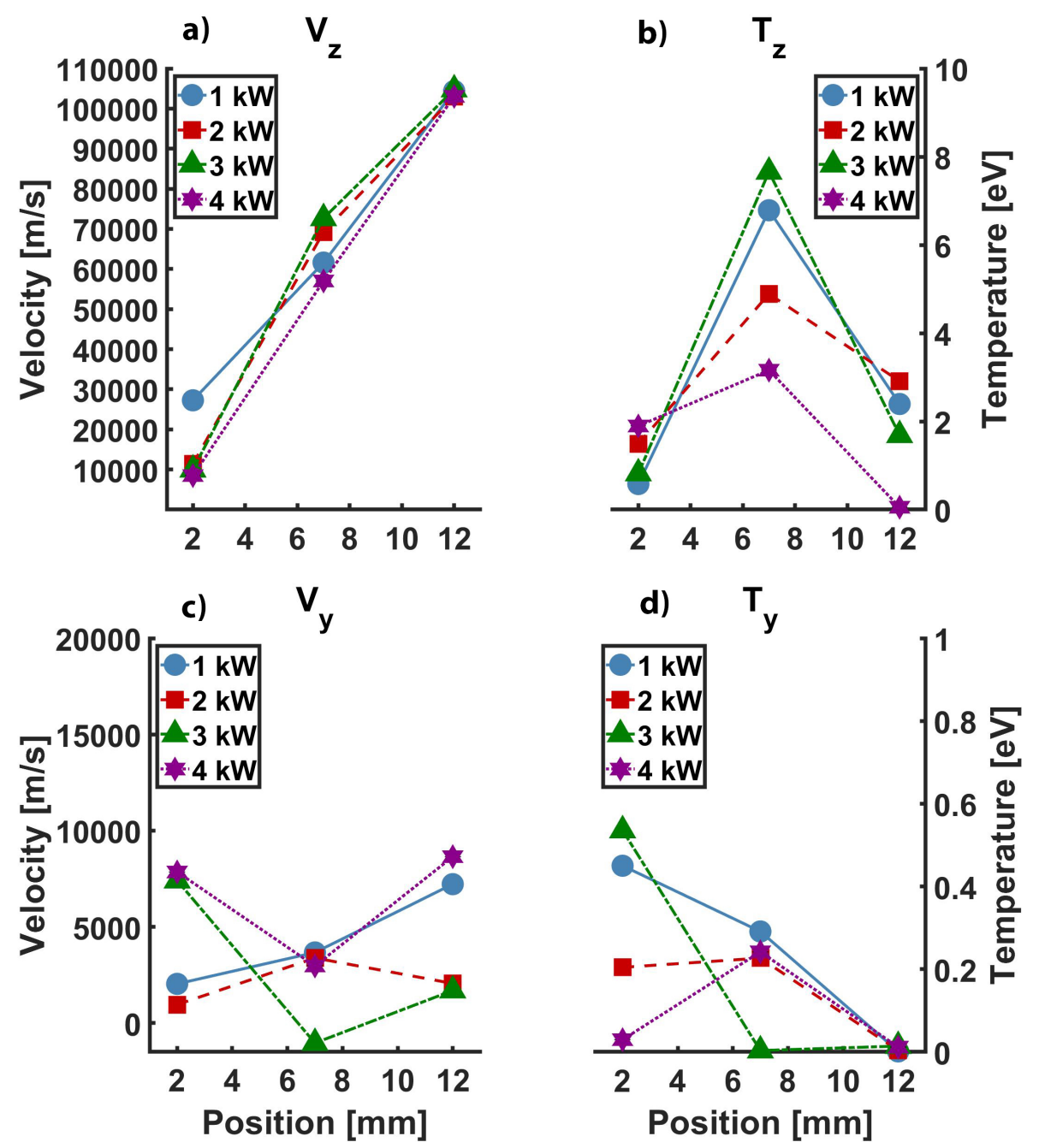

Figure 4.8: The velocity and temperature calculated for IVDFs outside the source with a $3000 \mathrm{~V}$ extraction potential. The ions heat significantly in $\hat{z}$, peaking at $z=7 \mathrm{~mm}$ and cool with distance in $\hat{y}$. The negative, then positive slopes in $v_{y}$ indicate $P_{f}=3 \mathrm{~kW}$ and $P_{f}=4 \mathrm{~kW}$ produce a focused beam near the middle of the measurement region.

lower ion signal at $P_{f}=1 \mathrm{~kW}$ and $P_{f}=2 \mathrm{~kW}$ introduce significant noise to the IVDFs, making it difficult to measure reliable ion properties at distances far from the source. This is also true for $P_{f}=3 \mathrm{~kW}$ at $z=12$, where the ion density is relatively low.

At $3000 \mathrm{~V}$ applied bias, shown in Fig. 4.8, the ion velocity in $\hat{z}$ increases uniformly for all input powers. The maximum speed obtained near the biased wafer is $\approx 105 \mathrm{~km} / \mathrm{s}$. A first order approximation for the predicted ion speed at $3000 \mathrm{~V}$ can be found assuming conservation of energy: $(1 / 2) m v_{z}^{2}=e U$, and solving for velocity 
gives $v_{z} \approx 120 \mathrm{~km} / \mathrm{s}$. While the discrepancy is still sizable, $\Delta v_{z}=-15 \mathrm{~km} / \mathrm{s}$, the two values are comparable. Given that the ions have high temperatures at 3000 $\mathrm{V}$, the plasma is collisional and energy is not necessarily conserved. Moreover the modification to the biased wafer affects the accelerating potential in additional to difficulty measuring $12 \mathrm{~mm}$ with the $2 \mathrm{D}$ optics, so the ions measured here have not accelerated through a full $3000 \mathrm{~V}$ potential.

Measurements for $P_{f}=1 \mathrm{~kW}$ and $P_{f}=2 \mathrm{~kW}$ in $\hat{y}$ at $3000 \mathrm{~V}$ have poor SNR but give generally positive slopes. For these powers, the Debye sheath will not be able to overcome the unipolar sheath and the meniscus should produce overfoucused beams. For $P_{f}=3 \mathrm{~kW}$ and $P_{f}=4 \mathrm{~kW}$, the slope is negative between $z=0$ and $z=7$, indicating a focusing beam. The negative value at $z=7$ is due to the large collection volume near the focal point measuring ions below the $y=0$ plane. Then between $z=7$ and $z=12$ the slope is positive, indicating that the measurements were taken on the far side of the focal point and the beam is therefore diverging. The same trend of focusing and diverging is shown for $P_{f}=4 \mathrm{~kW}$.

\subsection{Ion flow and temperature in the meniscus re- gion}

Measurements of the IVDF were taken near the aperture of the Applied Materials ion source. Data were obtained at a constant source power of $P_{f}=3 \mathrm{~kW}$ with an extraction potential $V_{b}$ between $0 \mathrm{~V}-3000 \mathrm{~V}$ at a constant pressure of $1 \mathrm{~m}$ Torr in an argon plasma. These measurements represent the first non-perturbative, localized measurements of the plasma meniscus region.

In the aperture region, between $z=-3 \mathrm{~mm}$ and $z=+1 \mathrm{~mm}$, IVDFs were obtained for different biases at constant rf power. These measurements covered $4 \mathrm{~mm}$ of depth near the aperture as well as the area of the aperture opening. Measurements 

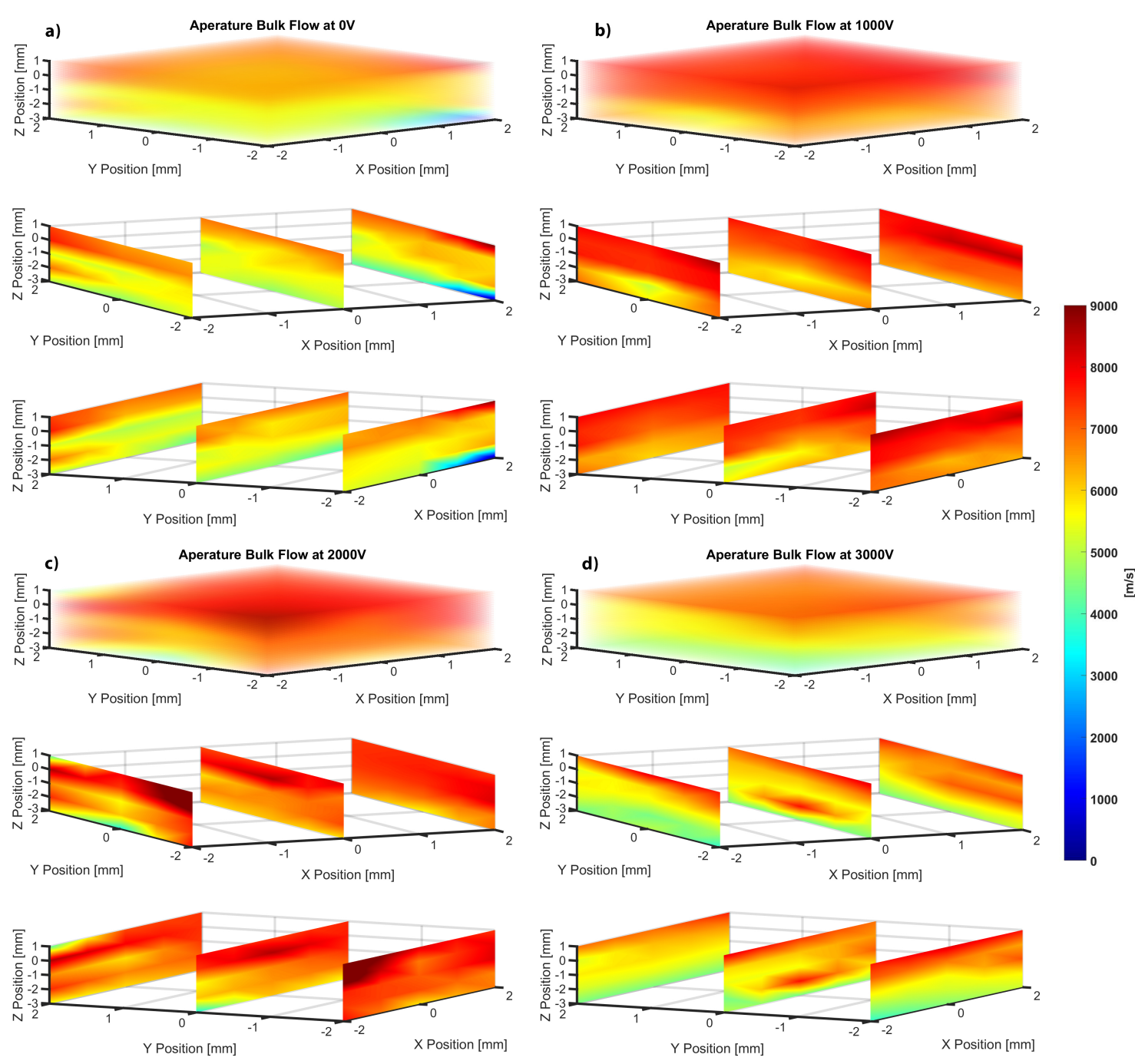

Figure 4.9: Ion flow through the aperture. A 3x3 grid, with measurements in $x$ and $y$ at $2 \mathrm{~mm}$ increments, was obtained for each $z$ position, then interpolated to create velocity contours. The velocity increases uniformly in the $z$ plane for increasing $z$ and potential for a) - c). d) Indicates a concave boundary at $z=-3$ in both the $x$ and $y$ plane.

were taken at $x=-2,0,2 \mathrm{~mm}$ and $y=-2,0,2 \mathrm{~mm}$ for each $z$ plane, giving a full view of the ion properties through the aperture. A constant power of $3 \mathrm{~kW}$ was chosen because it provided a suitable balance of LIF signal, scan time, and avoided the need to power cycle. The velocities from these scans are shown in Fig. 4.9 for each bias potential. The ion properties in this region are obtained from the moments of the distribution.

Figure 4.9a-c shows that the ion velocity is largely constant across each fixed $z$ plane. The velocity increases with increasing $z$ and applied potential for $V_{b}<2000$ 
$\mathrm{V}$. At a bias of $3000 \mathrm{~V}$, the ion velocity through the aperture is slower than both the $1000 \mathrm{~V}$ and $2000 \mathrm{~V}$ cases. Additionally, Fig. 4.9d shows slow ions in a concave shape at $z=-3$ for the $x=0$ and $y=0$ planes. There is also a consistent trend of higher flows at $x=-2 \mathrm{~mm}$ for Fig. $4.9 \mathrm{a}-\mathrm{c}$. Since this trend seems to be location dependent, it is most likely a geometric effect due to asymmetries in the aperture.

The ion temperature for the same region is given in Fig. 4.10. The measurements range from approximately $0.2 \mathrm{eV}$ to $0.7 \mathrm{eV}$. There is also an artificial "hot spot" at $z \geq 0 \mathrm{~mm}$ due to scatting of injected light off the extraction optics. There is no consistent spatial dependence of the ion temperature throughout the aperture region. However, the ion temperature does increase with potential up to $V_{b}=2000$ $\mathrm{V}$ by as much as $0.5 \mathrm{eV}$. This indicates that the ions close to the aperture are responding to the wafer's applied bias. If the ions are heating due to the presence of an electric field, it is expected that the peak ion temperature occurs at the largest $V_{b}$. However, ion temperature measured for $3000 \mathrm{~V}$ have colder temperatures than ion temperatures for $2000 \mathrm{~V}$.

This break from the trend at $V_{b}=3000 \mathrm{~V}$ is present throughout the data collected so far (Figs. $4.9-4.10$ ). The $3000 \mathrm{~V}$ case is naively expected to have the fastest and hottest ions, however, the velocity and temperature peak at $2000 \mathrm{~V}$. Arcing from the wafer to the source at $3000 \mathrm{~V}$ was observed, but this is a quick and very sporadic effect that occurred on a much shorter time scale than the scan time of the measurements. Since the trend appears across multiple input powers and confocal collection patterns, the effects at $3000 \mathrm{~V}$ are not from errors in measurements. Currently, due to the lack of previous investigations into the meniscus region, it is difficult to determine the cause of this effect with certainty. However, a reasonable conjecture is that at $3000 \mathrm{~V}$ the meniscus expands around the interior of the aperture optics. This causes ions accelerated by the Debye sheath to exit at unfavorable angles for extraction.

For all aperture measurements, the power is kept constant. Therefore, there 

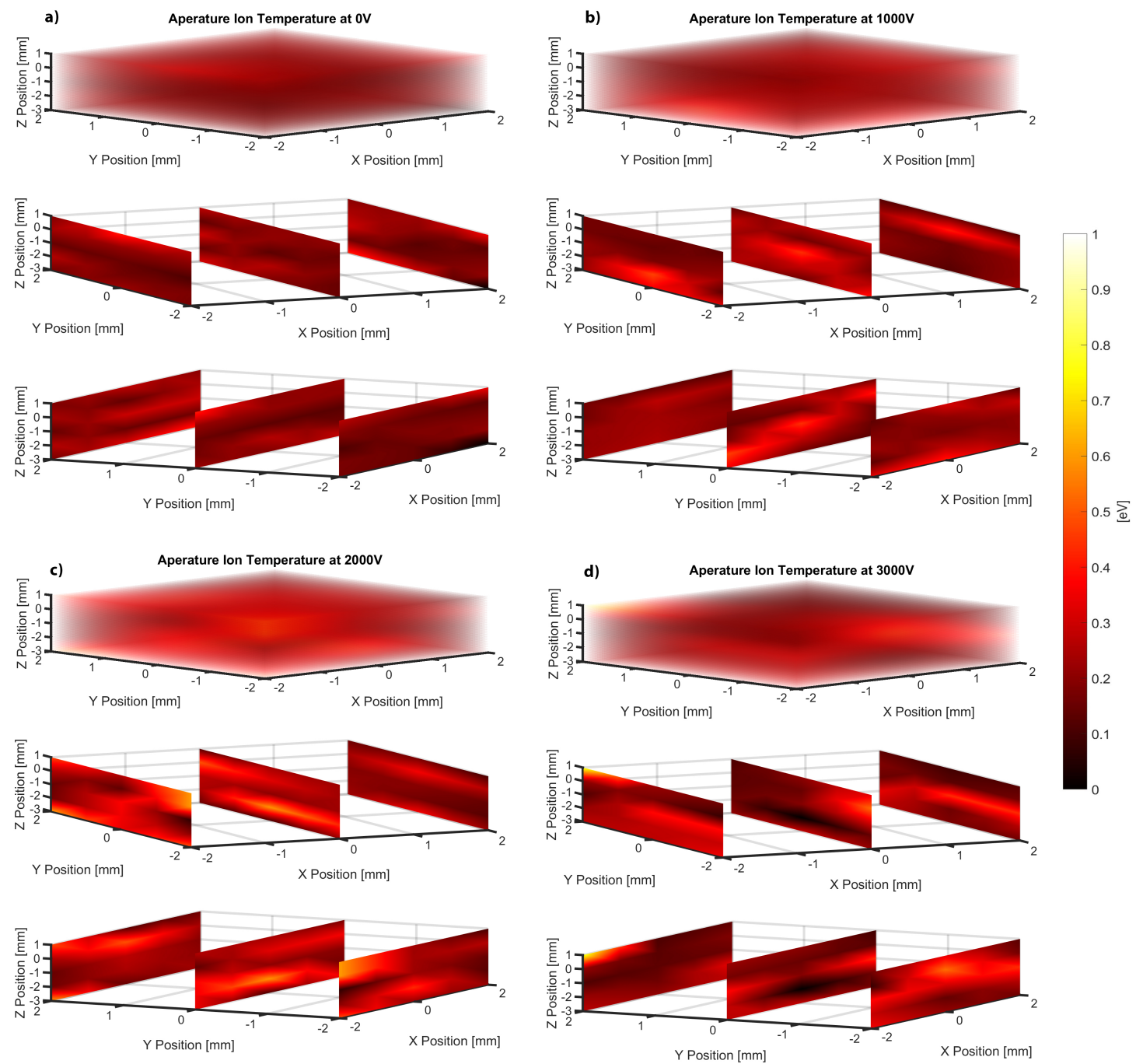

Figure 4.10: Ion temperature in the aperture region. Ions increase in heat with increase applied potential for a) - c). d) Has colder ions than the $1000 \mathrm{~V}$ case.

are no effects from variation in rf power (ion density, collisions, electron heating). Moreover, the ions are experiencing the biased potential between $0 \mathrm{~V}$ and $2000 \mathrm{~V}$, as evidenced by an increase in velocity and temperature. However, at $3000 \mathrm{~V}$ this plasma meniscus structure is suggested to be destroyed. This could be due to an "expansion" of the meniscus inside the aperture that results in an over-focused ion beam. The expanded meniscus, shown in Fig. 4.11, occurs at sufficiently strong applied potentials. Since the boundary of the meniscus acts as an electrostatic lens, ions will exit normal to the boundary, which in the case of an expanded meniscus are almost entirely in the transverse direction. When the meniscus is shallow the majority of the ions are extracted, but as the boundary gets pushed further into the 


\section{a)}
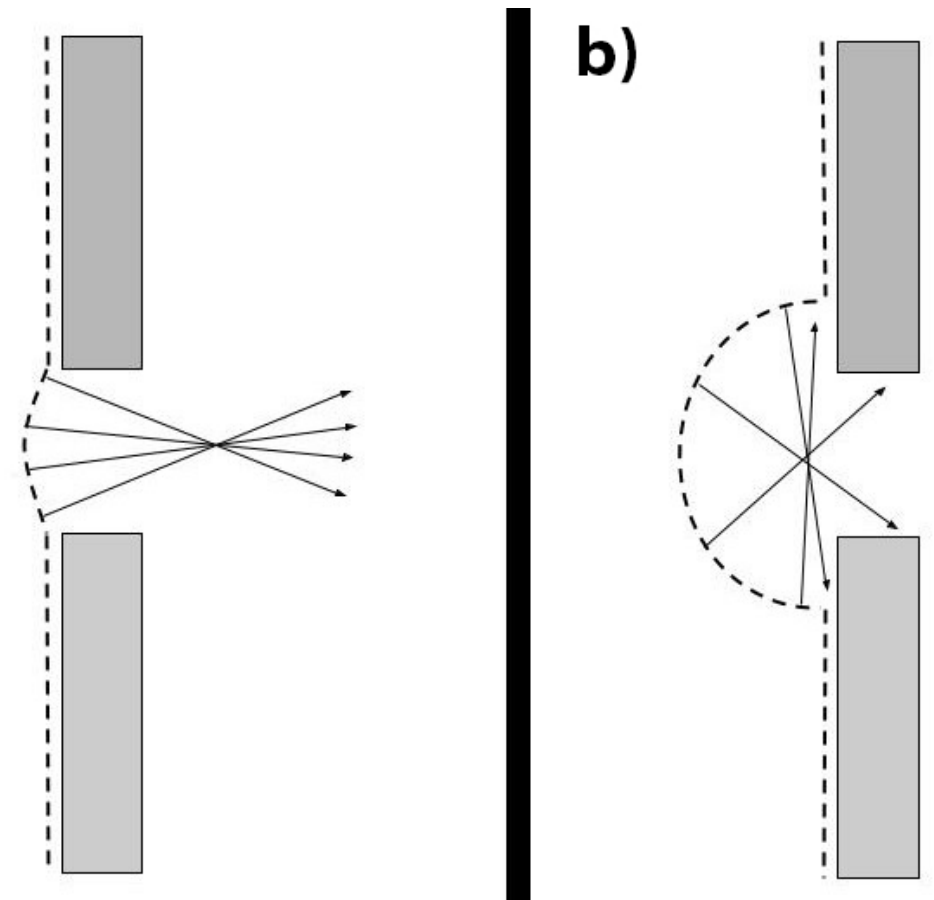

Figure 4.11: Two depictions of menisci with different radii of curvature. a) When the curvature is small, most of the ions can be extracted through the aperture. b) When the radius of curvature is large, the focal point can be inside the source and very few ions have a favorable extraction velocity.

source and expands, more of the ions will exit the Debye sheath with trajectories increasingly perpendicular to the direction of extraction. Therefore, the ions have almost no initial velocity in the direction of extraction, resulting in slower measured velocities than a shallower meniscus would produce.

\subsection{LIF Measurements Inside the Source}

LIF was used to obtain IVDFs inside the ICP source. This was facilitated by the use of the confocal optics system, which allowed for measurements along $\hat{z}$ inside and outside the source. Measurements were taken at $x=y=0$ to avoid clipping the collection cone on the aperture, maximizing the measurement depth in the source region. This allowed for a measurements range of $-25<z<1 \mathrm{~mm}$. Using the standard deviation in velocity and temperature from fiducial measurements in the source, measurements from the confocal apparatus has a total velocity uncertainty 

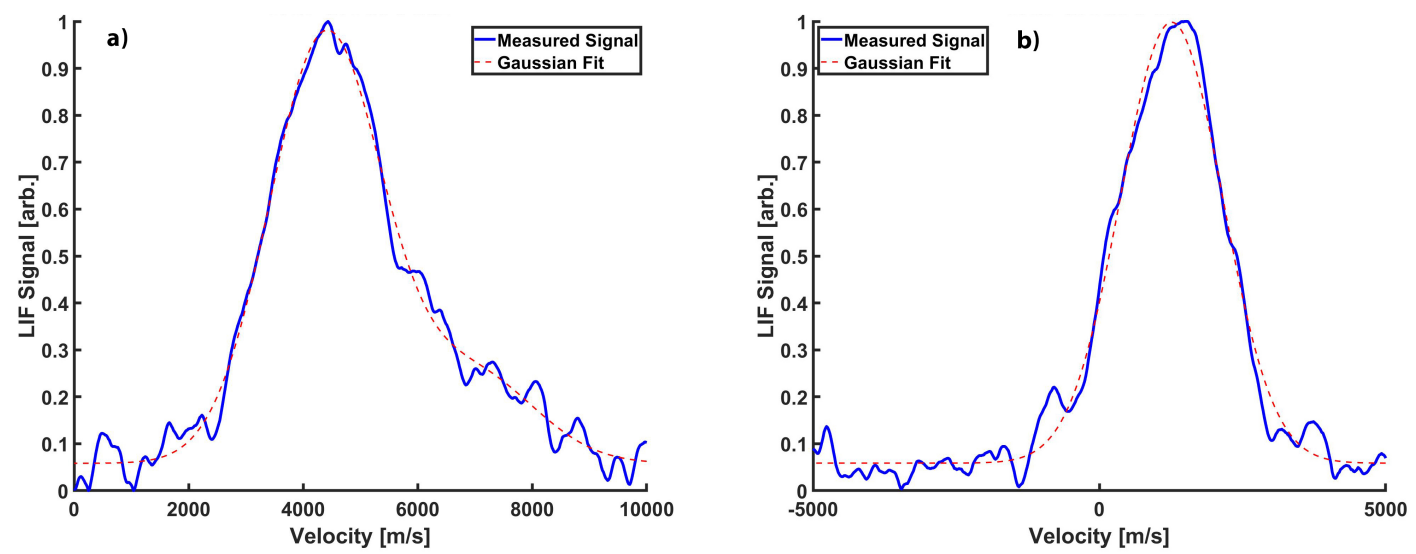

Figure 4.12: IVDFs obtained at $P_{f}=3 \mathrm{~kW}$ and $V_{b}=0 \mathrm{~V}$ a) $z=0 \mathrm{~mm}$ showing non-Maxwellian features formed by a double Gaussian and b) at $z=-25 \mathrm{~mm}$ with a Maxwellian lineshape.

of $\delta v_{\text {confocal }}= \pm 93 \mathrm{~m} / \mathrm{s}$ and a temperature uncertainty of $\delta T_{\text {confocal }}= \pm 0.02 \mathrm{eV}$.

Deep in the source, the IVDFs are Maxwellian but, for measurements closer to the aperture, the IVDFs have a non-Maxwellian feature. Figure 4.12 shows the difference in distribution between a measurement near the aperture and deep in the source. Mechanisms such as elastic or charge-exchange collisions may produce a high-energy tail [127]. Another possible cause of this feature is a second population of ions, close in velocity to the bulk distribution but slightly faster (Fig. 4.13). Assuming this is the mechanism responsible for the feature, a bi-Gaussian fit is used to obtain ion properties for both peaks. However, it is unclear what is producing this feature, which leaves room for debate in the appropriate analysis method. To characterize the ions in the following distributions, both analysis methods are applied for completeness.

\subsubsection{Bulk flow near the aperture from Two Population fits}

In order to compensate for the non-Maxwellian features in the IVDFs a two Gaussian fit was used. A bi-Gaussian is produced by adding a second term to Eqn. 3.2 such 

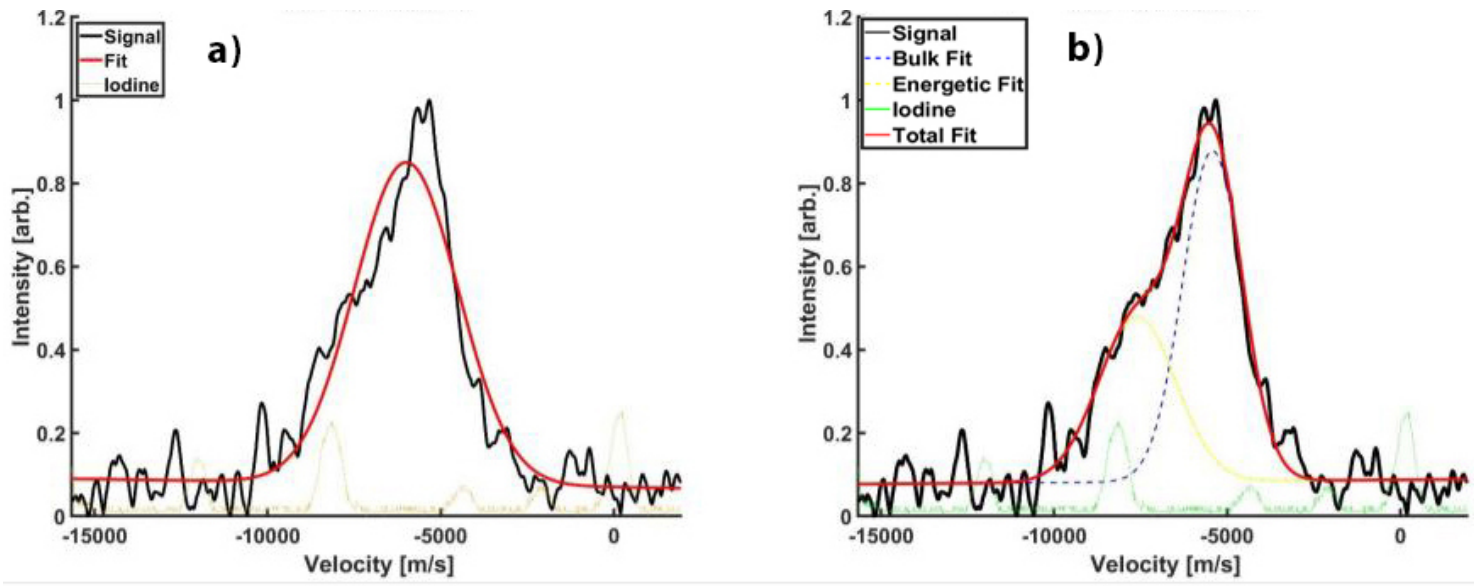

Figure 4.13: A comparison of the two fitting methods for the same IVDF obtained at $P_{f}=3 \mathrm{~kW}, V_{b}=1000 \mathrm{~V}$ and $z=0 \mathrm{~mm}$. a) A single Gaussian fit to the peak, giving $u=6000 \mathrm{~m} / \mathrm{s}$ and $T_{i}=0.93 \mathrm{eV}$. b) A fit with a bi-Gaussian distribution giving $u=5400 \mathrm{~m} / \mathrm{s}$ and $T_{i}=0.31 \mathrm{eV}$ for the bulk distribution and $u=7600 \mathrm{~m} / \mathrm{s}$ and $T_{i}=0.52 \mathrm{eV}$ for the faster population.

that

$$
I_{L I F}(v)=\alpha+C_{1} \exp \left[\frac{-\left(m / 2 k_{B}\right)\left(v-u_{1}\right)^{2}}{T_{1}}\right]+C_{2} \exp \left[\frac{-\left(m / 2 k_{B}\right)\left(v-u_{2}\right)^{2}}{T_{2}}\right],
$$

where $C$ is the normalization constant and the subscript 1 and 2 denote different populations. One Gaussian tracked the bulk population and the other was used to characterize the high-energy peak. However, due to how close the two peaks are and the lack of physical constraints for the high-energy peak, it is difficult to reliably fit the second population. This produces many possible velocities and temperatures that give a "good" overall fit and prevents both populations from being reliably characterized. Ion velocity of the bulk population, however, is reliably obtained if the fit constraints for the high-energy population are relaxed. This is because the bulk peak has a much more well defined peak and an example of this fit is shown in Fig. 4.13. In this manner, the ion velocity of the bulk peak was obtained for all IVDF measurements inside the source.

The velocity of the bulk distribution as a function of $z$ is shown in Fig. 4.14. The bulk ion velocity increases slowly deep in the source $(z \leq-10 \mathrm{~mm})$ and much 

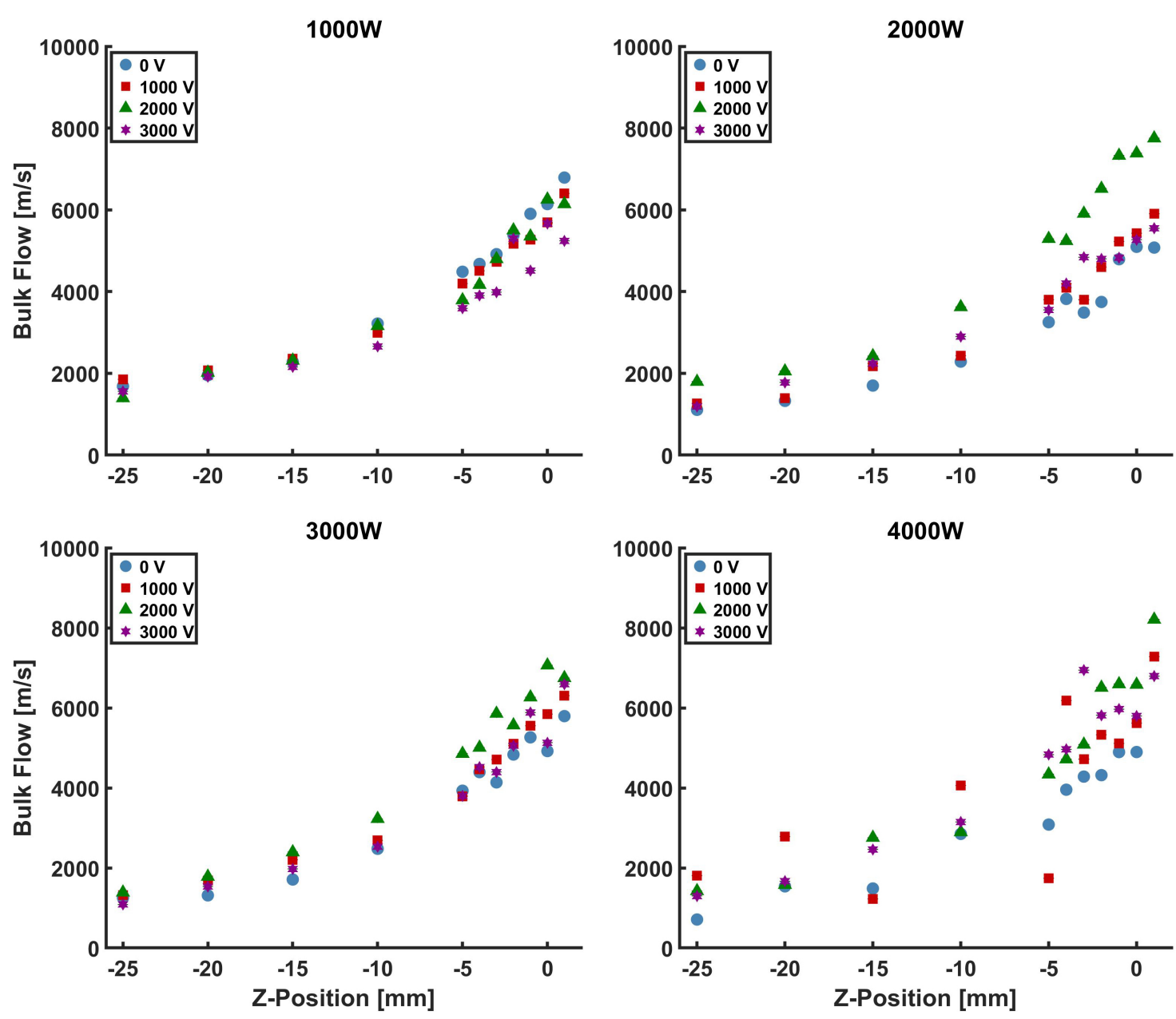

Figure 4.14: Bulk flow of the ion beam throughout the source for all powers and all biases. Bi-Gaussian fits were used to isolate the velocity of the bulk population (plotted here) from the high-energy population.

more quickly in the aperture region $(z \geq-5 \mathrm{~mm})$. The flow of the bulk population does not show any trend with increasing rf power, with the exception of overall faster flows at $4 \mathrm{~kW}$ (Fig. 4.14d). While a $2000 \mathrm{~V}$ extraction potential produces the fastest flows for Fig. 4.14b and c, the flows are largely independent of potential.

\subsubsection{IVDFs in the source}

As discussed above, using a bi-Gaussian fit makes the high-energy peak difficult to reliably isolate. Even when subtracting the bulk population from the IVDF, the low SNR of the high-energy peak prevents the calculation of fast ion properties. However, the source parameters' effects on the high-energy tail are apparent if the 

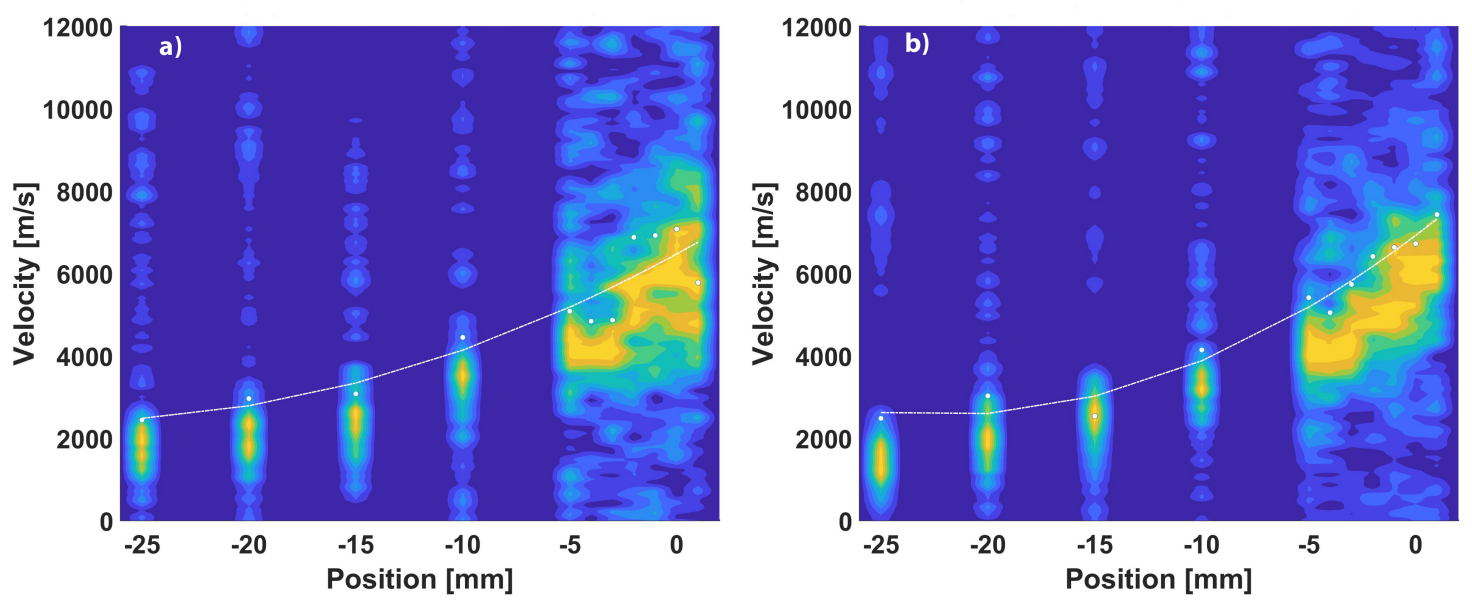

Figure 4.15: IVDFs comparing the signal at two different parameters with the highenergy boundary. a) Lower ion densities which create noise structures throughout the IVDF. b) Higher SNR, slightly reducing the appearance of noise.

full IVDF is viewed. In order to help distinguish the high-energy contribution from the bulk population, the half-width at half-maximum of the bulk distribution is calculated and added to the bulk flow. This location is calculated for each IVDF and then a line is fit to these points, as shown in Fig. 4.15. This line serves as an approximate boundary, above which the contribution is mostly from the high-energy peak and below which the signal is from the bulk population.

Figure 4.16 shows the IVDFs at all measurement locations inside the source. Each individual IVDF is normalized to unity so that the stronger signals in the source do not overpower the weaker ion signal near the aperture. Measurements with low SNR values, such as those for $1 \mathrm{~kW}$, are visible but have significant noise that obfuscate any high-energy features. Peaks in the source $(z \leq-10 \mathrm{~mm})$ have strong Maxwellian characteristics unlike distributions measured closer to the aperture.

The evolution of the high-energy peaks is traced by observing the IVDF signal above the high-energy separation line. Figure 4.16 shows the high-energy border for all measurements inside the source. In the aperture region ( $z \geq-5 \mathrm{~mm}$ ), as the potential increases up to $V_{b}=2000 \mathrm{~V}$, so too do the velocities of the highenergy contributions. This produces a trend where the slope of the density structure becomes steeper in the aperture region, Additionally, there is more signal in the high- 


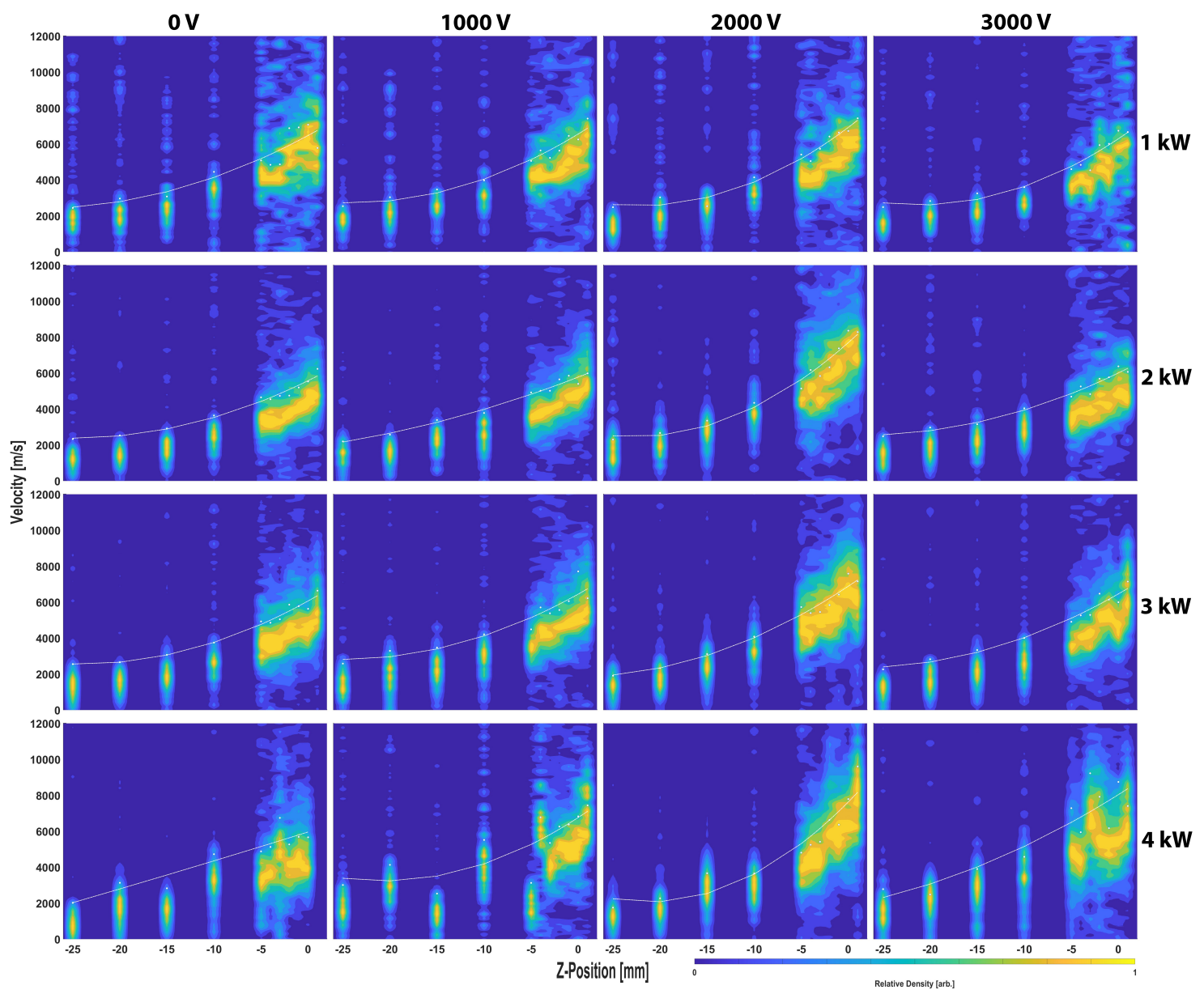

Figure 4.16: All IVDFs obtained in the source and near the aperture of REVAN with the confocal optical arrangement. Rows are constant powers and columns are constant biases. The dashed white line represents the border of the bulk population and the high energy contribution. Each IVDF is self normalized to one.

energy population with increasing bias relative to the bulk population. Furthermore, the high energy contribution drops off at $V_{b}=3000 \mathrm{~V}$, resulting in a lower average flow and temperature, similar to the meniscus measurements in Section 4.4.

Given the results shown in Fig. 4.9, Fig. 4.14, and Fig. 4.16, the high-energy peak is produced by ions created in the source that fall through the full potential of the Debye sheath. This is why the high-energy peak is dependent on the applied potential and the population appears fast because they are accelerated by the full potential. This means that the bulk population, and therefore the majority of extracted ions, are not controllable by the extraction optics. The presence of this bulk population is similar to IVDF models of the beam halo [128]. In negative ion sources, the beam halo is formed due to ions with velocities opposite the direction 
of extraction that fall into the extraction sheath [129]. This creates two populations with different focal properties. However, at sufficiently high biases $\left(V_{b}=3000 \mathrm{~V}\right)$ the high energy contribution is reduced due to the meniscus expansion, resulting in a more uniform extraction. The mechanism producing the ions in the beam halo is not determinable from the data obtained in this investigation.

\subsubsection{Ions heat near the extraction region}

The ion temperature is calculated from the second moment of the distribution function. Since the second moment is an integral over the entire distribution, this gives an effective ion temperature, $T_{\text {eff }}$. Figure 4.17 shows $T_{\text {eff }}$ for measurements inside the plasma source.

Far from the extraction optics $(z \leq-10 \mathrm{~mm})$ the ion temperature varies $\leq 0.1$ $\mathrm{eV}$ at all powers except $P_{f}=4 \mathrm{~kW}$. An rf power of $P_{f}=1 \mathrm{~kW}$ produces ion temperatures of $\sim 0.05 \mathrm{eV}$, which is near room temperature. However, as input power increases, the ion temperature deep in the source also increases. Since an increase in antenna power will increase the heating of electrons, this suggests that the electrons are collisionally coupled to the ions.

At $P_{f}=4 \mathrm{~kW}$, a maximum temperature of $1 \mathrm{eV}$ ions are measured near the extraction region. The high-energy population of ions are heated in a collisional presheath through collisions with neutrals [130]. Since this effect is dependent on collisions an increase in power, and therefore pressure, will increase the ion heating. Additionally, a higher extraction potential will increase the flow of ions in the highenergy population, producing hotter ions near the extraction region. However, this is not reflected in Fig. 4.17 since the temperature is an average and dominated by the bulk population's contribution. At best, the high-energy temperature is qualitatively determined to increase, shown in Fig. 4.16, by observing the width of the high-energy contribution in velocity space. However, due to the inability to fit 

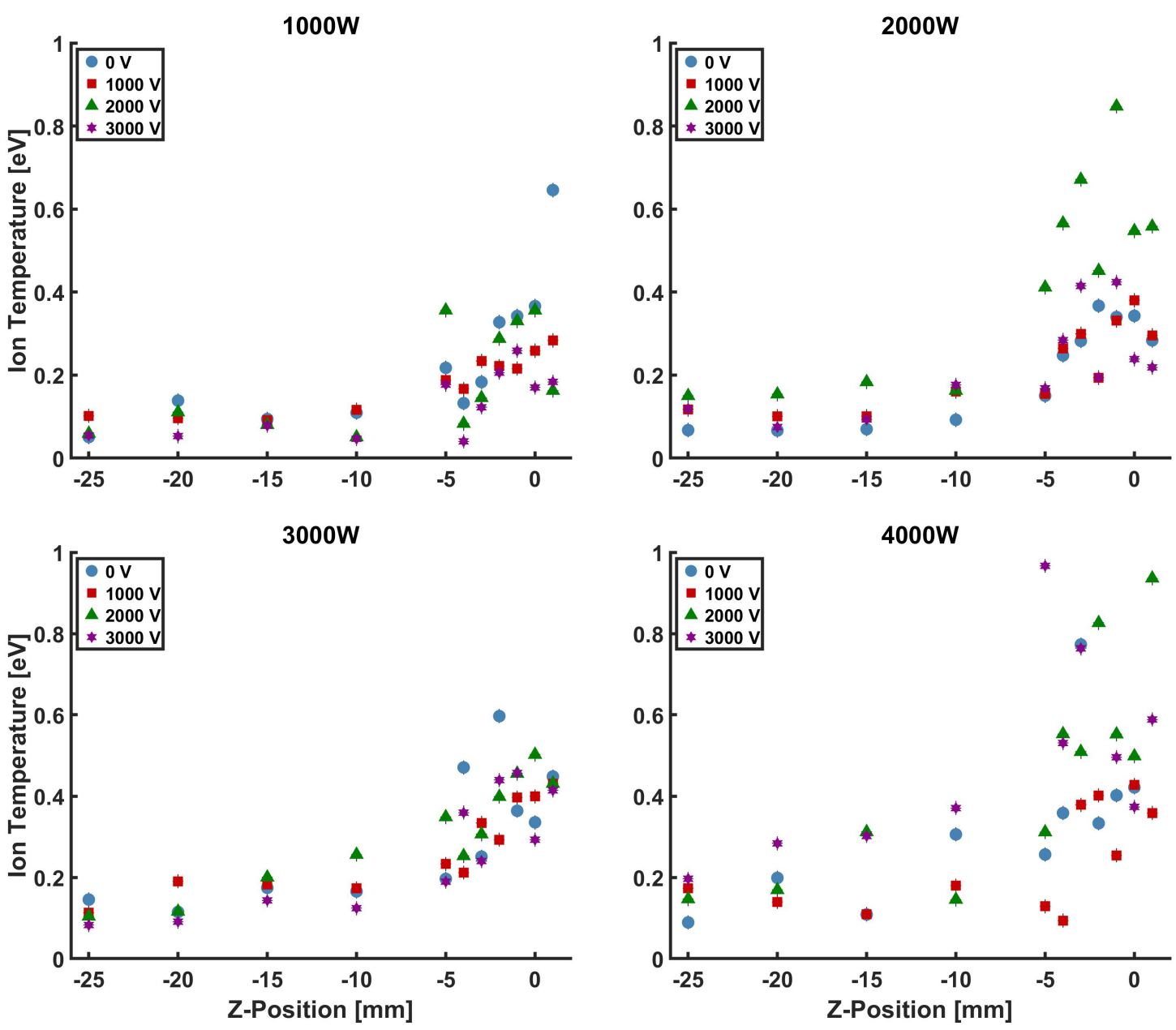

Figure 4.17: Effective ion temperature for all input powers and all biases. There is ion heating with increasing input power both in the source and especially near the aperture a) - c). d) The large fluctuation in data in $4 \mathrm{~kW}$ is due to the ion source heating during continuous operation.

a Gaussian to this population, exact temperatures are not well defined.

\subsection{Estimation of plasma density and properties from EEPFs}

A Langmuir probe was used to obtain EEPFs for the plasma produced by REVAN. As mentioned above, REVAN settled into a new equilibrium that prohibited $\mathrm{H}-$ mode operation before EEPFs could be measured. Therefore, the results described in this section cannot be directly compared to the previous sections in this chapter. 
Nevertheless, measurements were carried out in the interest of completeness. Since the ion source settled into an E-mode plasma, it was being sustained by capacitive coupling rather than inductive. This has a few consequences for the data, namely that the density and electron temperature will be lower [54,69,72].

\subsubsection{Electron Density}

The electron density was calculated from the electron saturation current given by Eqn. 3.20. Figure 4.18 shows the electron density along the extraction axis for different source powers. During these measurements there was no extraction potential applied, meaning that there is no plasma meniscus, only a Debye sheath.

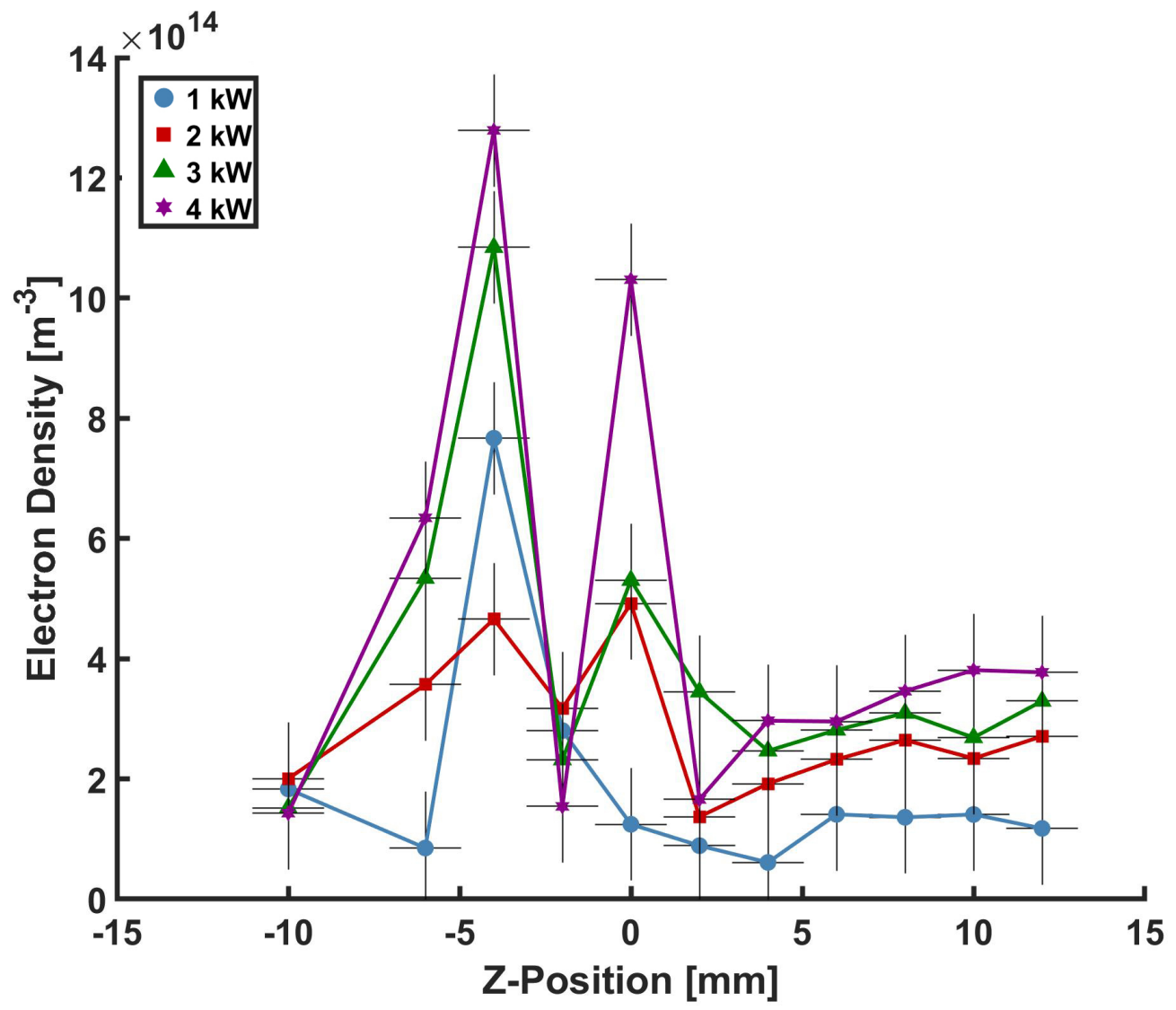

Figure 4.18: The electron density across the entire measurement domain for REVAN in an E-mode operation. Excluding $z=-2$, where the probe's collection sheath forms a continuous boundary with the Debye sheath, the electron density increases with increasing rf-power. 
The electron density ranges from $\approx 1.0 \times 10^{14} \mathrm{~m}^{-3}-1.3 \times 10^{15} \mathrm{~m}^{-3}$ with a large increase in the aperture region. The uncertainty in electron density was calculated from fiducial measurements to be $\delta n_{e}= \pm 9.3 \times 10^{13} \mathrm{~m}^{-3}$. These measurements were obtained inside the source where sheath rectification caused large errors in the current measurement (Section 3.3.3.2).

Just inside the aperture $(z=-2 \mathrm{~mm})$ there is a sharp decrease in electron density, most likely due to the fact that the probe is in the Debye sheath of the extraction optics. Excluding the measurements in the Debye sheath, the electron density increases with source power. This is expected since more input power will result in an increase in ionization and plasma density. At $z=2 \mathrm{~mm}$, however, all measurements are within error of each other.

\subsubsection{Electron Temperature}

The electron temperature calculated from the non-drifting Maxwellian method is shown in Fig. 4.19 with an uncertainty in temperature of $\delta T_{e}=0.17 \mathrm{eV}$. Like the electron density, the electron temperature on either side of the aperture is rf power dependent. There is also an inversion of the electron temperature dependence on source power close to extraction aperture. This is because in the Debye sheath, only electrons with sufficient energy will be able to overcome the sheath potential and reach the probe surface, and at low power the sheath is larger, requiring more energetic electrons. This spike is rather pronounced with electrons reaching temperatures of $32 \mathrm{eV}$. As power is increased, the inductive contribution is increased as well but this increase will be relatively small compared to the capacitive effects.

The Debye lengths at two different positions $(z=-2 \mathrm{~mm}, z=0 \mathrm{~mm})$ for the E-mode operating regime were calculated using Eqn. 1.5. These values are obtained from the densities and temperatures calculated in this section and are given in Table 4.1. The measurements at $z=0 \mathrm{~mm}$ range from $\lambda_{D}=0.71-3.14 \mathrm{~mm}$ with an 


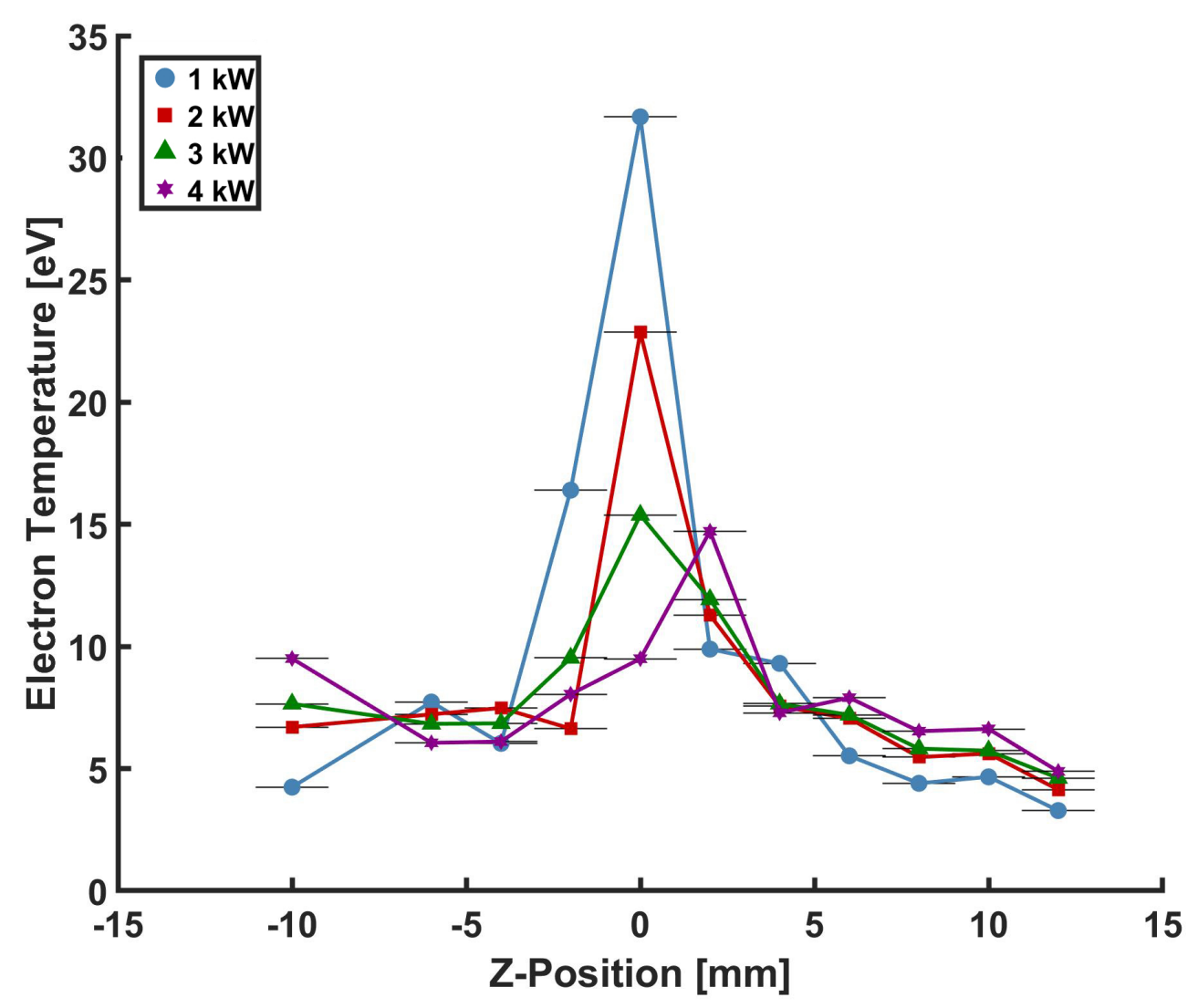

Figure 4.19: Electron temperature across REVAN's experimental domain. The electron temperature increases with increasing rf-power, except in the aperture region where the Debye sheath interferes with the probe's collection sheath.

increase in distance at lower rf power. This is expected since a lower density will produce a larger sheath. At $z=-2 \mathrm{~mm}$, which due to the length of the probe tip is more indicative of the aperture region, the values of the Debye length do not change as much with source parameters. In fact, with a propagating uncertainty from the Langmuir probe of $\delta \lambda_{D} \approx 0.31 \mathrm{~mm}$, all sheath lengths at $z=-2 \mathrm{~mm}$ are within error of one another. This means that there will be a continuous sheath between the extraction optics and the Langmuir probe and the data obtained at $-2<z<0$

\begin{tabular}{|ccc|}
\hline rf-power $(\mathrm{kW})$ & $\lambda_{D, z=0}(\mathrm{~mm})$ & $\lambda_{D, z=-2}(\mathrm{~mm})$ \\
\hline \hline 1 & 3.75 & 1.80 \\
2 & 1.60 & 1.07 \\
3 & 1.26 & 1.50 \\
4 & 0.71 & 1.70 \\
\hline
\end{tabular}

Table 4.1: The Debye length for different REVAN input powers at two locations in the aperture. 
$\mathrm{mm}$ is not indicative of the background plasma. 


\section{Chapter 5}

\section{Conclusion}

Ion beams have been studied for decades due to their essential use in systems like silicon wafer etching and doping and NBI. The majority of these studies have been focused on the ion properties after the beam has been extracted from the source. However, relatively little is known about the emitting boundary that forms these ion beams. This boundary, known as the plasma meniscus, is crucial to understand if semiconductor fabrication techniques are to advance and meet the demands of three-dimensional architecture.

Extraction optics were used to create an ion beam that is extracted at an angle. LIF measurements of the resulting angled beam were able to map the flow and structure of the beam as it expanded in a vacuum chamber with no externally applied biases. The beam angle was found to be $\approx-30^{\circ}$ with respect to normal of the extraction optics. This angle is less than naively expected given the geometry of the extraction optics. The ions also have a larger velocity in the $\hat{z}$-direction than in the $\hat{y}$-direction, indicating a large initial velocity inside the source in $\hat{z}$. Extraction optics such as these provide a new beam geometry for the doping and etching of semiconductors. Instead of having to tilt the wafer in order to expose trenches and walls to ion beams, the source can produce the angled beam. The angle is dependent on the geometry of the extraction optics, allowing for optimization through changes 
in the slit geometry. This angular control, along with a greater understanding of the plasma meniscus, helps to push manufacturing closer to realizing three-dimensional transistors.

The ion beam formed by an ICP in E-mode operation was investigated. While the measurements were not taken at the same conditions as the meniscus measurements, the LIF technique proved its capabilities in measuring focal points due to the plasma meniscus. Whether the ion beam was focused or diverging was determined by a combination of relative ion density, since a focal point should have a large density of ions, and the velocity of the ions in the $y$-direction, since transverse velocity decreases up to a focal point and increases after. Ion beams were only observed at source powers $P_{f} \geq 3 \mathrm{~kW}$ for a $3000 \mathrm{~V}$ extraction potential. At $0 \mathrm{~V}$ all ion beams were diffuse due to the lack of an extraction potential, and therefore a plasma meniscus. At $3000 \mathrm{~V}$, the focal point depended on the rf power with $P_{f}=4 \mathrm{~kW}$ producing a focal point further from the extraction optics than $P_{f}=3 \mathrm{~kW}$.

In this work, an investigation into the ion properties inside an inductively coupled plasma source was conducted in order to establish how source parameters affect the plasma meniscus and therefore the resulting ion beam. A graphite wafer was set up near the aperture to simulate a silicon wafer and create the extraction potential. Measurements were taken for a range of rf source powers $(1 \mathrm{~kW}, 2 \mathrm{~kW}, 3 \mathrm{~kW}, 4$ kW) and extraction biases (1000 V, 2000V, 3000V, 4000V). These measurements represent the first non-perturbative measurements of the ion velocity distribution functions inside a semiconductor ion source. Measurements in the aperture suggest the formation of a meniscus at $P_{f}=3 \mathrm{~kW}$ and $V_{b}=3000 \mathrm{~V}$ (Fig. 4.9d).

IVDFs obtained deep inside the source $(z \leq-10 \mathrm{~mm})$ show that the ions have a Maxwellian distribution. However, the ions also have a large velocity $\left(v_{z} \geq 1000\right.$ $\mathrm{m} / \mathrm{s}$ ) in this region. This velocity is too large to be from a pressure differential between the ion source and the vacuum chamber. Additionally, it is unlikely that the ions are in a collisional presheath because there is very little temperature increase 
when compared with measurements near the aperture. One possibility is that the ions are interacting with the induced field, similar to the anomalous skin effect, but to accurately describe this trend investigations much farther into the ICP source are needed to characterize the full ion acceleration.

Near the aperture there are two contributions to the ion signal. The highenergy contribution is from ions produced deep in the source that traverse the entire potential structure near the aperture. It remains to be determined how or where the bulk population ions are created. However, the bulk population is extracted in a manner similar to the beam halo found in $\mathrm{H}^{-}$sources. The beam halo is detrimental to extracted beams because it creates a second beam with different focal properties. However, in these negative ion sources, the beam halo is formed from surface ionization on the extraction optics, which create ions with velocities opposite the direction of extraction [131]. REVAN does not have surface produced ions so the mechanism for the production of beam halo ions in positive ion sources remains an open question.

In the aperture region the ion temperature and velocity in the aperture region increase for potential biases $0 \mathrm{~V}-2000 \mathrm{~V}$. At $3000 \mathrm{~V}$, however, the ions slow down and cool for all input powers (Fig. 4.16). Given that a constantly increasing extraction potential should create a constant increase in ion velocity, potentials at this strength are believed to be altering the meniscus topology. This is most likely due to the meniscus becoming large enough (or expanding) that the focal point of the beam is inside the aperture (Fig. 4.11). At $V_{b}=3000 \mathrm{~V}$, however, there is no high-energy contribution to the IVDFs. Therefore, the ion beam actually has better extraction properties despite having less velocity because there will only be one focal spot, from the bulk distribution.

The results described here provide new insights into how ions are extracted from ICPs and will hopefully help inform the next generation of semiconductor fabrication methods. However, this work also presents many new areas of ICPs that warrant 
further investigation. What happened to the meniscus structure at $3000 \mathrm{~V}$, and can a confocal optical arrangement achieve sufficient spatial localization to detail its topology? What is the actual acceleration that ions undergo on their way to the aperture? What is the mechanism that populates the beam halo? For future studies, TALIF is promising method to obtain ion velocity distributions, and thereby non-perturbative ion density measurements. 


\section{Appendix A}

\section{Mode Degradation}

The ICP's degradation from H-mode operation to E-mode operation occurred over the course of three measurements (9 minutes). The obtained IVDFs varied wildly from one to the next, despite being at the same spatial location and parameter setting, indicating a failure mode in the source. Upon revisiting fiducial measurement locations inside the source, it was found that the obtained IVDFs did not match previously acquired IVDFs. REVAN underwent extensive troubleshooting in an attempt to remedy this issue.

Based on visual observations, REVAN's plasma would start a deep purple, indicating an argon plasma, and slowly change to a paler, pink color. This gave rise to the possibility of a leak in the source. Since REVAN uses rubber gaskets, it could be possible that the heating of the source created a micro-leak, allowing contamination into the feed gas. Additionally, an oxygen plasma glows bright white, so a mixture of argon and oxygen could explain the new color of the plasma. All vacuum lines, gas bottles, and flanges were leak tested during operation and no leak was present.

Even with a magnetic cusp field, the plasma in REVAN is still capable of etching the aluminum chamber and the graphite extraction optics. This led to deposition of conductive impurities on the dielectric barrier, as shown in Fig. A.1. This can 


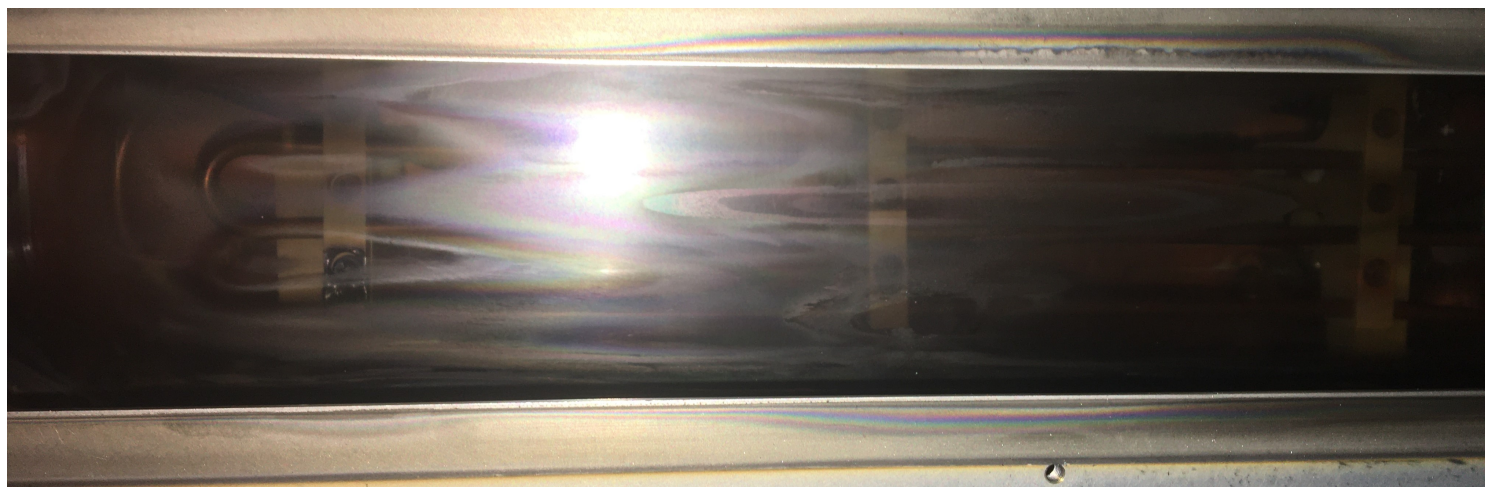

Figure A.1: Image of REVAN's dielectric window after E-mode degradation. The antenna can be seen on the other side, but is covered by deposition that will reduce the power transferred from the antenna to the ion source.

lead to a decrease in the effective power coupled into the plasma. Therefore, the dielectric was replaced with an identical dielectric barrier obtained from the same company. However, REVAN still could not access H-mode operations.

The last attempt to recover $\mathrm{H}$-mode operations was to completely re-wire the rf circuit. In addition to the rf generator reaching high temperatures, the circuitry inside REVAN's Faraday cage would also reach temperatures hot enough to melt plastic that was not actively cooled. Under the assumption of wire deterioration due to sustained operating temperatures, all connectors and wires were replaced. There was another antenna available, but with a different inductance value, number of turns, and area. Therefore, this new antenna would change the fundamental plasma generation in REVAN and it was not used. Despite new wiring the plasma continued to operate in E-mode.

With all other possibilities ruled out, it is likely that the auto-matching network failed. The rf generator's front display reads out the power forward $P_{f}$ and the reflected power $P_{r}$. In H-mode operations, the maximum reflected power through the antenna circuit was $12 \mathrm{~W}$ at $P_{f}=4 \mathrm{~kW}$. After the switch to E-mode, the rf generator would continue to read similar or lower $P_{r}$ values. However, Eqn. 2.5 shows that for H-mode onset, all that is needed is current above a certain threshold to be delivered to the antenna. However, no amount of $P_{f}$ was able to access H-mode operations. 
Unfortunately, the inner workings of this auto-matching network are a blackbox. The matching was manually adjusted by the external grounding capacitor. However, no amount of tuning the grounding capacitor could bring the auto-matching network out of the stable E-mode the auto-match preferred. In previous H-mode operation the grounding capacitor had very limited values that generated a plasma. Therefore it is believed that the auto-matching network failed and was not delivering enough current through to sustain an H-mode discharge. 


\section{Appendix B}

\section{Current Density}

Given the limitations in determining the plasma density and electron temperature, an accurate current density cannot be calculated for this investigation. However, it is worth revisiting the current density theory of Section 1.3.2 and its applicability in determining the distance of the unipolar sheath, and therefore ion emitting surface. An approximate electron density can be calculated by assuming an electron temperature of $9 \mathrm{eV}$ inside the aperture at $P_{f}=3 \mathrm{~kW}$. This is a reasonable assumption based on Fig. 4.19 and the linear scaling of electron temperature with power presented for H-mode plasmas given in Reference [110]. Given that the ion temperature is known, as well as a neutral pressure, the ideal gas law can be used to give a plasma density by assuming $90 \%$ ionization and using the equation,

$$
P=n k_{B} T_{a v g}
$$

where $P$ is the neutral pressure and the average particle temperature is given by $T_{\text {avg }}=\left(T_{\text {eff }}+T_{e}\right) / 2$. This yields an approximate density of $n_{e} \approx 1 \times 10^{17} \mathrm{~m}^{-} 3$. This value is above the typical H-mode onset density and is in the range of densities for plasma processing sources $[70,75]$.

As discussed in Section 1.3.1, ions that are accelerated by the Debye sheath and 


\begin{tabular}{|cccc|}
\hline Applied Bias $(\mathrm{V})$ & $j\left(A / m^{2}\right)$ & $j_{c_{s}}\left(A / m^{2}\right)$ & $j_{C L}\left(A / m^{2}\right)$ \\
\hline \hline 0 & 64 & & - \\
1000 & 88 & 75 & $3.42 \times 10^{17}$ \\
2000 & 104 & & $9.67 \times 10^{17}$ \\
3000 & 72 & & $1.78 \times 10^{18}$ \\
\hline
\end{tabular}

Table B.1: Comparison of theoretically predicted current densities $\left(j_{C L}, j_{c_{s}}\right)$ with experimentally calculated current density $j$.

enter the unipolar sheath at their ion sound speed. The derivation for the Bohm criterion assumes that the ions are much colder than the electrons $\left(T_{i} \ll T_{e}\right)$ and are therefore ignored. In this limit Eqn. 1.11 takes the form,

$$
c_{s}=\sqrt{\frac{T_{e}}{m_{i}}}
$$

An ion sound speed of $c_{s} \approx 4600 \mathrm{~m} / \mathrm{s}$ is calculated from Eqn. B.2. Using this speed, the ion sound speed current density is $j_{c_{s}} \approx 75 \mathrm{~A} / \mathrm{m}^{2}$. The actual ion current density $j$ is calculated from the ion velocities given in Fig. 4.14 at a distance of $z=-2$. Lastly, the Child-Langmuir current density, which is independent of electron temperature and density, is calculated. All three current densities are given in Table B.1 for all extraction potentials.

While this is an approximate calculation for $j$ and $j_{c s}$, it is a much more reliable result for $j_{C L}$ since it only depends on the applied bias and distance. The ChildLangmuir law gives an upper limit on the allowable current, which in this case is unreasonably large. The estimated plasma properties used here would have to be off by many, many orders of magnitude to explain this discrepancy. Rather, the over estimation of the Child-Langmuir law shows that Eqn. 1.20 is not applicable for low energy ion source such as REVAN and will not give correct estimations of the distance to the ion emitting surface. 


\section{Bibliography}

[1] M. A. Lieberman and A. J. Lichtenberg, Principles of plasma discharges and materials processing. John Wiley \& Sons, 2 ed., 2005. 0-471-72001-1.

[2] W. Leahy, V. Barron, M. Buggy, T. Young, A. Mas, F. Schue, T. McCabe, and M. Bridge, "Plasma surface treatment of aerospace materials for enhanced adhesive bonding," The Journal of Adhesion, vol. 77, no. 3, pp. 215-249, 2001.

[3] I. Junkar, U. Cvelbar, and M. Lehockỳ, "Plasma treatment of biomedical materials," Materiali in tehnologije, 2011.

[4] M. Mollah, R. Schennach, J. Patscheider, S. Promreuk, and D. L. Cocke, "Plasma chemistry as a tool for green chemistry, environmental analysis and waste management," Journal of Hazardous Materials, vol. 79, no. 3, pp. 301$320,2000$.

[5] I. Adamovich, S. Baalrud, A. Bogaerts, P. Bruggeman, M. Cappelli, V. Colombo, U. Czarnetzki, U. Ebert, J. Eden, P. Favia, et al., "The 2017 plasma roadmap: Low temperature plasma science and technology," Journal of Physics D: Applied Physics, vol. 50, no. 32, p. 323001, 2017.

[6] G. E. Moore, "Cramming more components onto integrated circuits," IEEE solid-state circuits society newsletter, vol. 11, no. 3, pp. 33-35, 2006.

[7] G. E. Moore et al., "Progress in digital integrated electronics," in Electron devices meeting, vol. 21, pp. 11-13, Maryland, USA, 1975. 
[8] D. B. Graves, "Plasma processing," IEEE transactions on Plasma Science, vol. 22, no. 1, pp. 31-42, 1994.

[9] D. K. Coultas and J. H. Keller, "Radio frequency induction/multipole plasma processing tool.” US Patent, 1989. US5304279A.

[10] J. Jang, H.-S. Kim, W. Cho, H. Cho, J. Kim, S. I. Shim, J.-H. Jeong, B.-K. Son, D. W. Kim, J.-J. Shim, et al., "Vertical cell array using tcat (terabit cell array transistor) technology for ultra high density NAND flash memory," in 2009 Symposium on VLSI Technology, pp. 192-193, IEEE, 2009.

[11] P. K. Chu, S. Qin, C. Chan, N. W. Cheung, and L. A. Larson, "Plasma immersion ion implantation - a fledgling technique for semiconductor processing," Materials Science and Engineering: R: Reports, vol. 17, no. 6-7, pp. 207-280, 1996.

[12] L. Rubin and J. Poate, "Ion implantation in silicon technology," Industrial Physicist, vol. 9, no. 3, pp. 12-15, 2003.

[13] N. Sakudo, K. Tokiguchi, H. Koike, and I. Kanomata, "Microwave ion source for high-current implanter," Review of Scientific Instruments, vol. 49, no. 7, pp. 940-943, 1978.

[14] S. Felch, Z. Fang, B.-W. Koo, R. Liebert, S. Walther, and D. Hacker, "Plasma doping for the fabrication of ultra-shallow junctions," Surface and Coatings Technology, vol. 156, no. 1-3, pp. 229-236, 2002.

[15] S. Felch, C. Hobbs, J. Barnett, H. Etienne, J. Duchaine, M. Rodgers, S. Bennett, F. Torregrosa, Y. Spiegel, and L. Roux, "Plasma doping of silicon fin structures," in 11th International Workshop on Junction Technology (IWJT), pp. 22-25, IEEE, 2011.

[16] B. Mizuno, I. Nakayama, N. Aoi, M. Kubota, and T. Komeda, "New doping method for sub half-micron trench sidewalls by using an electron cyclotron 
resonance plasma," Applied Physics Letters, vol. 53, no. 21, pp. 2059-2061, 1988.

[17] S. Felch, E. Arevalo, S. Walther, Z. Fang, B. Koo, R. Liebert, D. Lenoble, and A. Grouillet, "Comparison of ultra-shallow junctions with plad and beamline implantation," in 2000 International Conference on Ion Implantation Technology Proceedings. Ion Implantation Technology-2000 (Cat. No. 00EX432), pp. 488-491, IEEE, 2000.

[18] P. Verdonck, "Plasma etching," Oficina de Microfabricao: Projeto e Construcao de CI's MOS, Swart, JW, Ed., Campinas (Sao Paulo, Brazil): UNICAMP, p. 9, 2006.

[19] C. Cardinaud, M.-C. Peignon, and P.-Y. Tessier, "Plasma etching: principles, mechanisms, application to micro-and nano-technologies," Applied Surface Science, vol. 164, no. 1-4, pp. 72-83, 2000.

[20] R. A. Gottscho, C. W. Jurgensen, and D. Vitkavage, "Microscopic uniformity in plasma etching," Journal of Vacuum Science \& Technology B: Microelectronics and Nanometer Structures Processing, Measurement, and Phenomena, vol. 10, no. 5, pp. 2133-2147, 1992.

[21] J. Parasuraman, A. Summanwar, F. Marty, P. Basset, D. E. Angelescu, and T. Bourouina, "Deep reactive ion etching of sub-micrometer trenches with ultra high aspect ratio," Microelectronic engineering, vol. 113, pp. 35-39, 2014.

[22] M. Abdelrahman, "Factors enhancing production, extraction and focusing of positive ion beams," Ain Shams Engineering Journal, vol. 3, no. 1, pp. 71-78, 2012.

[23] R. Kakoschke, R. E. Kaim, P. F. van der Meulen, and J. Westendorp, "Trench sidewall implantation with a parallel scanned ion beam," IEEE transactions on electron devices, vol. 37, no. 4, pp. 1052-1056, 1990. 
[24] K. Wieczerzak, A. Priebe, I. Utke, and J. Michler, "Practical aspects of focused ion beam time-of-flight secondary ion mass spectrometry analysis enhanced by fluorine gas coinjection," Chemistry of Materials, vol. 33, no. 5, pp. 1581-1593, 2021.

[25] N. B. Moore, W. Gekelman, P. Pribyl, Y. Zhang, and M. J. Kushner, "2dimensional ion velocity distributions measured by laser-induced fluorescence above a radio-frequency biased silicon wafer," Physics of Plasmas, vol. 20, no. 8, p. 083506, 2013.

[26] B. Knuffman, A. V. Steele, and J. J. McClelland, "Cold atomic beam ion source for focused ion beam applications," Journal of Applied Physics, vol. 114, no. 4, p. 044303, 2013.

[27] N. Gavrilov and A. Kamenetskikh, "Experimental study of the effect of processes in the range of ion beam propagation on results of electrical measurements of beam current," Zhurnal Tekhnicheskoj Fiziki, vol. 76, no. 6, pp. 32$37,2006$.

[28] P. Veltri, E. Sartori, P. Agostinetti, D. Aprile, M. Brombin, G. Chitarin, N. Fonnesu, K. Ikeda, M. Kisaki, H. Nakano, et al., "Ion beam transport: modelling and experimental measurements on a large negative ion source in view of the ITER heating neutral beam," Nuclear Fusion, vol. 57, no. 1, p. 016025, 2016.

[29] F. F. Chen et al., Introduction to plasma physics and controlled fusion. Springer, 2 ed., 1984. 978-0306413322.

[30] T. Boyd and J. Sanderson, The Physics of Plasmas. Cambridge University Press, 2003. 0521459125.

[31] I. A. Kotelnikov and V. T. Astrelin, "Theory of a plasma emitter of positive ions," Physics-Uspekhi, vol. 58, no. 7, p. 701, 2015. 
[32] D. Bohm, "Minimum ionic kinetic energy for a stable sheath," The Characteristics of Electrical Discharges in Magnetic Fields, pp. 77-86, 1949.

[33] C. Child, "Discharge from hot CaO," Physical Review (Series I), vol. 32, no. 5, p. 492, 1911.

[34] O. Sutherland, J. Keller, M. Irzyk, and R. Boswell, "Comparison between experiment and two simulation strategies for the extraction of focused ion beams," Review of Scientific Instruments, vol. 75, no. 7, pp. 2379-2386, 2004.

[35] S. K. Nam, V. M. Donnelly, and D. J. Economou, "Particle-In-Cell simulation of ion flow through a hole in contact with plasma," IEEE Transactions on Plasma Science, vol. 33, no. 2, pp. 232-233, 2005.

[36] B. Soliman, M. Abdelrahman, A. Helal, and F. Abdelsalam, "Simulation of ion beam extraction and focusing system," Chinese Physics $C$, vol. 35, no. 1, p. $83,2011$.

[37] S. Lawrie, D. Faircloth, A. Letchford, C. Gabor, and J. Pozimski, "Plasma meniscus and extraction electrode studies of the ISIS H- ion source," Review of Scientific Instruments, vol. 81, no. 2, p. 02A707, 2010.

[38] K. Miyamoto, S. Okuda, S. Nishioka, and A. Hatayama, "Effect of basic physical parameters to control plasma meniscus and beam halo formation in negative ion sources," Journal of Applied Physics, vol. 114, no. 10, p. 103302, 2013.

[39] D. Ćirić, A. Ash, B. Crowley, I. Day, S. Gee, L. Hackett, D. Homfray, I. Jenkins, T. Jones, D. Keeling, et al., "Performance of upgraded jet neutral beam injectors," Fusion Engineering and Design, vol. 86, no. 6-8, pp. 509-512, 2011.

[40] W. Jinfang, W. Bin, and H. Chundong, "Simulation on heating and current drive using neutral beam injection with variable injection angle on east," Plasma Science and Technology, vol. 12, no. 3, p. 289, 2010. 
[41] I. Goumiri, C. W. Rowley, S. Sabbagh, D. Gates, M. Boyer, S. Gerhardt, E. Kolemen, and J. Menard, "Simultaneous feedback control of plasma rotation and stored energy on NSTX-U using neoclassical toroidal viscosity and neutral beam injection," Physics of Plasmas, vol. 24, no. 5, p. 056101, 2017.

[42] J.-L. Wei, L.-Z. Liang, C.-C. Jiang, Y.-H. Xie, C.-D. Hu, J. Li, Y.-M. Gu, Y.-Q. Chen, J.-Y. Li, and M.-S. Wu, "Beam optics study of a negative ion source for neutral beam injection application at ASIPP," Fusion Engineering and Design, vol. 117, pp. 93-99, 2017.

[43] K. Miyamoto, S. Okuda, S. Nishioka, and A. Hatayama, "Effect of basic physical parameters to control plasma meniscus and beam halo formation in negative ion sources," Journal of Applied Physics, vol. 114, no. 10, p. 103302, 2013.

[44] S. Nishioka, K. Miyamoto, S. Okuda, I. Goto, A. Hatayama, and A. Fukano, "Study of plasma meniscus and beam halo in negative ion sources using three dimension in real space and three dimension in velocity space particle in cell model," Review of Scientific Instruments, vol. 85, no. 2, p. 02A737, 2014.

[45] K. Miyamoto, S. Okuda, A. Hatayama, M. Hanada, and A. Kojima, "Study of beam optics and beam halo by integrated modeling of negative ion beams from plasma meniscus formation to beam acceleration," Applied Physics Letters, vol. 102, no. 2, p. 023512, 2013.

[46] J. Shalf, "The future of computing beyond Moore's law," Philosophical Transactions of the Royal Society A, vol. 378, no. 2166, p. 20190061, 2020.

[47] N. Kofuji, M. Mori, and T. Nishida, "Uniform lateral etching of tungsten in deep trenches utilizing reaction-limited NF3 plasma process," Japanese Journal of Applied Physics, vol. 56, no. 6S2, p. 06HB05, 2017. 
[48] M. F. Fatahilah, K. Strempel, F. Yu, S. Vodapally, A. Waag, and H. S. Wasisto, "3D GaN nanoarchitecture for field-effect transistors," Micro and Nano Engineering, vol. 3, pp. 59-81, 2019.

[49] H. Tanaka, M. Kido, K. Yahashi, M. Oomura, R. Katsumata, M. Kito, Y. Fukuzumi, M. Sato, Y. Nagata, Y. Matsuoka, et al., "Bit cost scalable technology with punch and plug process for ultra high density flash memory," in 2007 IEEE Symposium on VLSI Technology, pp. 14-15, IEEE, 2007.

[50] S.-H. Chen, H.-T. Lue, Y.-H. Shih, C.-F. Chen, T.-H. Hsu, Y.-R. Chen, Y.H. Hsiao, S.-C. Huang, K.-P. Chang, C.-C. Hsieh, et al., "A highly scalable 8-layer vertical gate 3D NAND with split-page bit line layout and efficient binary-sum MiLC (minimal incremental layer cost) staircase contacts," in 2012 International Electron Devices Meeting, pp. 2-3, IEEE, 2012.

[51] C. T. Gabriel and J. P. McVittie, "How plasma etching damages thin gate oxides," Solid State Technology, vol. 35, no. 6, pp. 81-88, 1992.

[52] W. Hittorf, "Ueber die electricitaetsleitung der gase," Annalen der Physik, vol. 257 , no. 1, pp. 90-139, 1884.

[53] M. A. Lieberman and R. A. Gottscho, "Design of high-density plasma sources for materials processing," in Physics of Thin Films, vol. 18, pp. 1-119, Elsevier, 1994.

[54] J.-K. Lee, H.-C. Lee, and C.-W. Chung, "E-H mode transition in inductively coupled plasma using Ar, O2, N2, and mixture gas," Current Applied Physics, vol. 11, no. 5, pp. S149-S153, 2011.

[55] V. Demidov, C. DeJoseph Jr, and A. Kudryavtsev, "Ionization kinetics and E$\mathrm{H}$ mode transition in a noble gas, low-pressure pulsed ICP discharge," Plasma Sources Science and Technology, vol. 13, no. 4, p. 600, 2004.

[56] J. Hopwood, "Review of inductively coupled plasmas for plasma processing," Plasma Sources Science and Technology, vol. 1, no. 2, p. 109, 1992. 
[57] J. Gudmundsson and M. A. Lieberman, "Magnetic induction and plasma impedance in a cylindrical inductive discharge," Plasma Sources Science and Technology, vol. 6, no. 4, p. 540, 1997.

[58] M. Tuszewski, "Enhanced radio frequency field penetration in an inductively coupled plasma," Physical Review Letters, vol. 77, no. 7, p. 1286, 1996.

[59] Y. Hua, J. Song, Z. Hao, G. Zhang, and C. Ren, "Characteristics of a dual-radio-frequency cylindrical inductively coupled plasma," Contributions to Plasma Physics, vol. 59, no. 7, p. e201800029, 2019.

[60] T. Czerwiec and D. Graves, "Mode transitions in low pressure rare gas cylindrical ICP discharge studied by optical emission spectroscopy," Journal of Physics D: Applied Physics, vol. 37, no. 20, p. 2827, 2004.

[61] I. El-Fayoumi, I. Jones, and M. Turner, "Hysteresis in the E-to H-mode transition in a planar coil, inductively coupled rf argon discharge," Journal of Physics D: Applied Physics, vol. 31, no. 21, p. 3082, 1998.

[62] R. Piejak, V. Godyak, and B. Alexandrovich, "A simple analysis of an inductive RF discharge," Plasma Sources Science and Technology, vol. 1, no. 3, p. $179,1992$.

[63] J. H. Keller, J. C. Forster, and M. S. Barnes, "Novel radio-frequency induction plasma processing techniques," Journal of Vacuum Science $\&$ Technology A: Vacuum, Surfaces, and Films, vol. 11, no. 5, pp. 2487-2491, 1993.

[64] M. Turner, "Collisionless electron heating in an inductively coupled discharge," Physical Review Letters, vol. 71, no. 12, p. 1844, 1993.

[65] V. Godyak, R. Piejak, and B. Alexandrovich, "Electron energy distribution function measurements and plasma parameters in inductively coupled argon plasma," Plasma Sources Science and Technology, vol. 11, no. 4, p. 525, 2002. 
[66] M. A. Lieberman and V. A. Godyak, "From fermi acceleration to collisionless discharge heating," IEEE Transactions on Plasma Science, vol. 26, no. 3, pp. 955-986, 1998.

[67] U. Kortshagen, N. Gibson, and J. Lawler, "On the e-h mode transition in rf inductive discharges," Journal of Physics D: Applied Physics, vol. 29, no. 5, p. 1224, 1996.

[68] M.-H. Lee and C.-W. Chung, "On the E to H and H to E transition mechanisms in inductively coupled plasma," Physics of Plasmas, vol. 13, no. 6, p. 063510, 2006.

[69] K. Suzuki, K. Nakamura, H. Ohkubo, and H. Sugai, "Power transfer efficiency and mode jump in an inductive RF discharge," Plasma Sources Science and Technology, vol. 7, no. 1, p. 13, 1998.

[70] J. H. Keller, "Inductive plasmas for plasma processing," Plasma Sources Science and Technology, vol. 5, no. 2, p. 166, 1996.

[71] N. Yoon, S. Kim, C. Chang, and D.-I. Choi, "One-dimensional solution for electron heating in an inductively coupled plasma discharge," Physical Review $E$, vol. 54, no. 1, p. 757, 1996.

[72] N. Kang and F. Gaboriau, "Simple modelling of the E-H mode transition and hysteresis in low pressure argon ICP discharges for direct comparison with experiments," Journal of Physics D: Applied Physics, vol. 44, no. 44, p. $442001,2011$.

[73] M. Turner and M. Lieberman, "Hysteresis and the E-to-H transition in radiofrequency inductive discharges," Plasma Sources Science and Technology, vol. 8, no. 2, p. 313, 1999.

[74] MKS, MWH-100 Auto-matching Network, 1998. 
[75] J. Hopwood, "Planar RF induction plasma coupling efficiency," Plasma Sources Science and Technology, vol. 3, no. 4, p. 460, 1994.

[76] J. Gosselin, S. Thakur, S. Sears, J. McKee, E. Scime, and G. Tynan, "Overestimation of mach number due to probe shadow," Physics of Plasmas, vol. 23, no. 7, p. 073519, 2016.

[77] R. Stern and J. Johnson III, "Plasma ion diagnostics using resonant fluorescence," Physical Review Letters, vol. 34, no. 25, p. 1548, 1975.

[78] T. Kajiwara, T. Shinkawa, K. Uchino, M. Masuda, K. Muraoka, T. Okada, M. Maeda, S. Sudo, and T. Obiki, "Application of two-photon-excited laserinduced fluorescence to atomic hydrogen measurements in the edge region of high-temperature plasmas," Review of Scientific Instruments, vol. 62, no. 10, pp. 2345-2349, 1991.

[79] J. Bokor, R. Freeman, J. White, and R. Storz, "Two-photon excitation of the n=3 level in H and D atoms," Physical Review A, vol. 24, no. 1, p. 612, 1981.

[80] A. Bailey III, R. Stern, and P. Bellan, "Measurement of coherent drift-wave ion-fluid velocity field when ion dynamics are stochastic," Physical Review Letters, vol. 71, no. 19, p. 3123, 1993.

[81] F. M. Levinton and F. Trintchouk, "Visualization of plasma turbulence with laser-induced fluorescence," Review of Scientific Instruments, vol. 72, no. 1, pp. 898-905, 2001.

[82] M. Zintl and R. McWilliams, "Improved optical tomography device," Review of Scientific Instruments, vol. 65, no. 8, pp. 2574-2579, 1994.

[83] D. S. Thompson, Three-dimensional multispecies distribution functions in a plasma boundary with an oblique magnetic field. PhD thesis, West Virginia University, Morgantown, WV, 2018. 
[84] A. M. Keesee, Neutral Density Profiles in Argon Helicon Plasmas. PhD thesis, West Virginia University, Morgantown, WV, 2006.

[85] A. M. Keesee, E. E. Scime, and R. F. Boivin, "Laser-induced fluorescence measurements of three plasma species with a tunable diode laser," Review of Scientific Instruments, vol. 75, no. 10, pp. 4091-4093, 2004.

[86] A. M. Keesee and E. Scime, "Neutral density profiles in argon helicon plasmas," Plasma Sources Science and Technology, vol. 16, no. 4, p. 742, 2007.

[87] J. Amorim, G. Baravian, and J. Jolly, "Laser-induced resonance fluorescence as a diagnostic technique in non-thermal equilibrium plasmas," Journal of Physics D: Applied Physics, vol. 33, no. 9, p. R51, 2000.

[88] C. B. Wharton and D. M. Slager, "Microwave determination of plasma density profiles," Journal of Applied Physics, vol. 31, no. 2, pp. 428-430, 1960.

[89] M. E. Galante, Two-photon absorption laser induced fluorescence measurements of neutral density in helicon plasma. West Virginia University, 2013.

[90] D. B. Elliott, Two photon absorption laser induced fluorescence for fusion class plasmas. West Virginia University, 2016.

[91] T. E. Steinberger, Single and Multi-photon Laser Induced Fluorescence for Electric Thruster and Fusion Applications. PhD thesis, West Virginia University, Morgantown, WV, Dec 2020.

[92] M. J. Goeckner, J. Goree, and T. E. Sheridan, "Laser-induced fluorescence characterization of a multidipole filament plasma," Physics of Fluids B: Plasma Physics, vol. 3, no. 10, pp. 2913-2921, 1991.

[93] R. Bovin, "Spectroscopy system and basic spectroscopy diagnostics for the HELIX and LEIA plasma devices," Tech. Rep. PL-046, West Virginia University, 2000. 
[94] C. Franck, J. Kline, and R. Spangler, "First order perturbed velocity distribution theory and measurement," Tech. Rep. PL-047, West Virginia University, 2000.

[95] X. Sun, E. Scime, M. Miah, S. Cohen, and F. Skiff, "Measurement of asymmetric optical pumping of ions accelerating in a magnetic-field gradient," Physical Review Letters, vol. 93, no. 23, p. 235002, 2004.

[96] R. Bovin, "Study of the different line broadening mechanisms for the laser induced fluorescence diagnostic of the HELIX and LEIA plasmas," Tech. Rep. PL-039, West Virginia University, 1998.

[97] H. R. Griem, Principles of plasma spectroscopy. No. 2, Cambridge University Press, 2005. 9780511524578.

[98] L. Maissel, "Stark effect in argon II," JOSA, vol. 48, no. 11, pp. 853-856, 1958.

[99] V. Gavrilenko, H. Kim, T. Ikutake, J. Kim, Y. Choi, M. Bowden, and K. Muraoka, "Measurement method for electric fields based on stark spectroscopy of argon atoms," Physical Review E, vol. 62, no. 5, p. 7201, 2000.

[100] D. S. Thompson, T. E. Steinberger, A. M. Keesee, and E. E. Scime, "Laser induced fluorescence of Ar-I metastables in the presence of a magnetic field," Plasma Sources Science and Technology, vol. 27, no. 6, p. 065007, 2018.

[101] G. Severn, D. Edrich, and R. McWilliams, "Argon ion laser-induced fluorescence with diode lasers," Review of Scientific Instruments, vol. 69, no. 1, pp. $10-15,1998$.

[102] M. Goeckner and J. Goree, "Laser-induced fluorescence measurement of plasma ion temperatures: Corrections for power saturation," Journal of Vacuum Science \& Technology A: Vacuum, Surfaces, and Films, vol. 7, no. 3, pp. 977-981, 1989. 
[103] W. Demtröder, Laser spectroscopy 1: basic principles. Springer, 2014. 978-3642-53859-9.

[104] E. B. Saloman, "Energy levels and observed spectral lines of ionized argon, Ar II through Ar XVIII," Journal of Physical and Chemical Reference Data, vol. 39, no. 3, p. 033101, 2010.

[105] F. Chu, R. Hood, and F. Skiff, "Measurement of wave-particle interaction and metastable lifetime using laser-induced fluorescence," Physics of Plasmas, vol. 26, no. 4, p. 042111, 2019.

[106] Y.-K. Lee and C.-W. Chung, "Ionization in inductively coupled argon plasmas studied by optical emission spectroscopy," Journal of Applied Physics, vol. 109, no. 1, p. 013306, 2011.

[107] X. Sun, C. Biloiu, R. Hardin, and E. E. Scime, "Parallel velocity and temperature of argon ions in an expanding, helicon source driven plasma," Plasma Sources Science and Technology, vol. 13, no. 3, p. 359, 2004.

[108] F. Chu and F. Skiff, "Determining metastable ion lifetime and history through wave-particle interaction," Physical Review Letters, vol. 122, no. 7, p. 075001, 2019.

[109] A. Phelps and J. Molnar, "Lifetimes of metastable states of noble gases," Physical Review, vol. 89, no. 6, p. 1202, 1953.

[110] V. Godyak, "Electrical and plasma parameters of ICP with high coupling efficiency," Plasma Sources Science and Technology, vol. 20, no. 2, p. 025004, 2011.

[111] M. Tadokoro, H. Hirata, N. Nakano, Z. L. Petrović, and T. Makabe, "Twodimensional density distribution of metastable atoms in an inductively coupled plasma in Ar," Physical Review E, vol. 58, no. 6, p. 7823, 1998. 
[112] S. DeVore, A. Gauthier, J. Levy, and C. Singh, "Improving student understanding of lock-in amplifiers," American Journal of Physics, vol. 84, no. 1, pp. 52-56, 2016.

[113] D. S. Thompson, M. F. Henriquez, E. E. Scime, and T. N. Good, "Confocal laser induced fluorescence with comparable spatial localization to the conventional method," Review of Scientific Instruments, vol. 88, no. 10, p. 103506 , 2017.

[114] M. F. Henriquez, D. S. Thompson, A. J. Jemiolo, and E. E. Scime, "Demonstration of confocal laser induced fluorescence at long focal lengths," Review of Scientific Instruments, vol. 89, no. 10, p. 10D127, 2018.

[115] S. Humphries, Charged particle beams. Courier Corporation, 2013. 9780486498683.

[116] I. Langmuir and H. Mott-Smith, "Langmuir probe technique," Gen. Elec. Rev, vol. 27 , no. 449 , p. $70,1924$.

[117] H. M. Mott-Smith and I. Langmuir, "The theory of collectors in gaseous discharges," Physical Review, vol. 28, no. 4, p. 727, 1926.

[118] V. Godyak and V. Demidov, "Probe measurements of electron-energy distributions in plasmas: what can we measure and how can we achieve reliable results?," Journal of Physics D: Applied Physics, vol. 44, no. 23, p. 233001, 2011.

[119] A. Garscadden and K. G. Emeléus, "Notes on the effect of noise on langmuir probe characteristics," Proceedings of the Physical Society (1958-1967), vol. 79, no. 3 , p. 535,1962 .

[120] I. D. Sudit and F. F. Chen, "RF compensated probes for high-density discharges," Plasma Sources Science and Technology, vol. 3, no. 2, p. 162, 1994. 
[121] I. H. Hutchinson, "Principles of plasma diagnostics," Plasma Physics and Controlled Fusion, vol. 44, no. 12, p. 2603, 2002.

[122] N. Hershkowitz, "How Langmuir probes work," Plasma diagnostics, vol. 1, pp. 113-183, 1989.

[123] V. Demidov, S. V. Ratynskaia, and K. Rypdal, "Electric probes for plasmas: The link between theory and instrument," Review of Scientific Instruments, vol. 73 , no. 10, pp. 3409-3439, 2002.

[124] F. F. Chen, "Langmuir probe diagnostics," in Mini-Course on Plasma Diagnostics, IEEEICOPS meeting, Jeju, Korea, 2003.

[125] R. Perkins, J. Hosea, M. Jaworski, R. Bell, N. Bertelli, G. Kramer, L. Roquemore, G. Taylor, and J. Wilson, "The role of rectified currents in far-field RF sheaths and in SOL losses of HHFW power on NSTX," Nuclear Materials and Energy, vol. 12, pp. 283-288, 2017.

[126] Lake Shore Cryotronics, Model 455 DSP Gaussmeter, 2014.

[127] H. Wang, V. S. Sukhomlinov, I. D. Kaganovich, and A. S. Mustafaev, "Simulations of ion velocity distribution functions taking into account both elastic and charge exchange collisions," Plasma Sources Science and Technology, vol. 26, no. 2, p. 024001, 2017.

[128] M. Salewski, B. Geiger, A. S. Jacobsen, M. García-Muñoz, W. Heidbrink, S. B. Korsholm, F. Leipold, J. Madsen, D. Moseev, S. K. Nielsen, et al., "Measurement of a $2 \mathrm{~d}$ fast-ion velocity distribution function by tomographic inversion of fast-ion d-alpha spectra," Nuclear Fusion, vol. 54, no. 2, p. 023005, 2014.

[129] K. Miyamoto, S. Okuda, and A. Hatayama, "Meniscus and beam halo formation in a tandem-type negative ion source with surface production," Applied Physics Letters, vol. 100, no. 23, p. 233507, 2012. 
[130] A. Meige, O. Sutherland, H. B. Smith, and R. W. Boswell, "Ion heating in the presheath," Physics of Plasmas, vol. 14, no. 3, p. 032104, 2007.

[131] K. Miyamoto, S. Nishioka, I. Goto, A. Hatayama, M. Hanada, A. Kojima, and J. Hiratsuka, "Analysis of the beam halo in negative ion sources by using $3 \mathrm{~d} 3 \mathrm{v}$ pic code," Review of Scientific Instruments, vol. 87, no. 2, p. 02B124, 2016. 